\title{
AUTOMATED PRUNING OF GREENHOUSE INDETERMINATE TOMATO PLANTS
}

\author{
A Thesis \\ presented to \\ the Faculty of California Polytechnic State University, \\ San Luis Obispo
}

\author{
In Partial Fulfillment \\ of the Requirements for the Degree \\ Master of Science in Computer Science
}

by

Joey Angeja

June 2018 
(c) 2018

Joey Angeja

ALL RIGHTS RESERVED 


\section{COMMITTEE MEMBERSHIP}

TITLE: Automated Pruning of Greenhouse Indeterminate Tomato Plants

AUTHOR: Joey Angeja

DATE SUBMITTED: June 2018

COMMITTEE CHAIR: Jane Zhang, Ph.D.

Professor of Electrical Engineering

COMMITTEE MEMBER: Bo Liu, Ph.D.

Professor of BioResource and Agricultural Engineering

COMMITTEE MEMBER: Lynne Slivovsky, Ph.D.

Professor of Computer and Electrical Engineering

COMMiTTEe MEMBER: Christian Eckhardt, Ph.D.

Professor of Computer Science and Software Engineering 


\begin{abstract}
Automated Pruning of Greenhouse Indeterminate Tomato Plants
\end{abstract}

Joey Angeja

Pruning of indeterminate tomato plants is vital for a profitable yield and it still remains a manual process. There has been research in automated pruning of grapevines, trees, and other plants, but tomato plants have yet to be explored. Wage increases are contributing to the depleting profits of greenhouse tomato farmers. Rises in population are the driving force behind the need for efficient growing techniques. The major contribution of this thesis is a computer vision algorithm for detecting greenhouse tomato pruning points without the use of depth sensors. Given an up-close 2-D image of a tomato stem with the background excluded, the algorithm proposed in this work can detect and mark the tomato suckers. 


\section{ACKNOWLEDGMENTS}

Thanks to:

- My parents, Joe and Liz, for supporting me through everything. Nothing would have been achievable without your love and support.

- My sisters, Katrina and Marissa, for unconditional love throughout my life. And for being my source of writer's block relief.

- Claudia Morales, you always pushed me to strive for success.

- My friends (Scott Jorgens, Spencer Jemes, Cameron Wariner, Jesse Bluestein, Alex Liaw, Shane Ashmore, Devin Strouse, Josh Jeter, Ricky Lang, Andrew Kim) for making my college experience unforgettable and urging me to always strive for success.

- Evan Low, for the innovative ideas.

- Dr. Zhang, for her support and ideas throughout my thesis.

- Dr. Liu, for the inspiration of the project. 


\section{TABLE OF CONTENTS}

Page

LIST OF TABLES . . . . . . . . . . . . . . . . . . ix

LIST OF FIGURES . . . . . . . . . . . . . . . . . . . . . CHAPTER

1 Introduction . . . . . . . . . . . . . . . . . 1

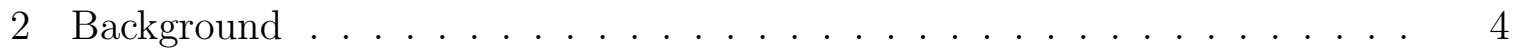

2.1 Greenhouse Indeterminate Tomatoes . . . . . . . . . . . . . . . . 4

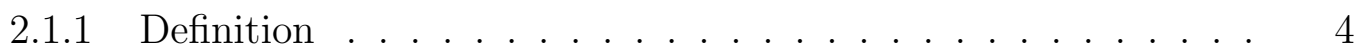

2.1.2 Pruning Points . . . . . . . . . . . . . . 5

2.2 Leveraged Technologies . . . . . . . . . . . . . . . . . 6

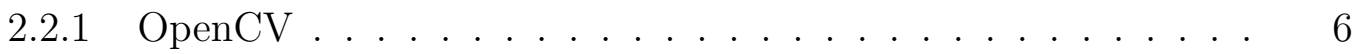

2.2 .2 NumPy ......................... 7

2.2 .3 SciKit ........................... 7

2.3 Depth ........................... 7

2.4 Scope ............................... 7

3 Related Work . . . . . . . . . . . . . . . . . . 9

3.1 Grapevine Pruning . . . . . . . . . . . . . . . . . . . 9

3.1.1 Image Preprocessing . . . . . . . . . . . . . . . . . . . 10

3.1 .2 Identifying Canes . . . . . . . . . . . . . . . . 11

3.1.3 Identifying Buds . . . . . . . . . . . . . . . 11

3.1.4 Identifying Pruning Points . . . . . . . . . . . . . . . . . . 12

3.1.5 Identifying the Canopy . . . . . . . . . . . . . . . . 13

3.2 Orchard Pruning . . . . . . . . . . . . . . . 14

3.2 .1 Data Acquisition . . . . . . . . . . . . . . 15

3.2.2 Obtaining 3D Point Cloud . . . . . . . . . . . . . . . 16

3.2.3 Trunk Localization . . . . . . . . . . . . . . . . 17

3.2.4 3D Model fitting to 3D Point Cloud . . . . . . . . . . . 18

3.2.5 Diameter Measurement . . . . . . . . . . . . . . . . . 19

3.3 Relevance . . . . . . . . . . . . . . . . . . . . . . . 19 
4 Initial Work . . . . . . . . . . . . . . . . . . . . . . . . . 21

4.1 Skeleton Classification . . . . . . . . . . . . . . . . 21

4.1 .1 Hough Line Transform . . . . . . . . . . . . . . . . . 21

$4.1 .2 \quad$ Kmeans Clustering . . . . . . . . . . . . . . . . . . . . 22

4.1 .3 Direct Neighbor Approach . . . . . . . . . . . . . . . . . 22

4.1.4 Joining Branch Segments . . . . . . . . . . . . . . . 23

4.2 Finding and Identifying the Main Stem . . . . . . . . . . . 24

4.3 Identifying Suckers . . . . . . . . . . . . . . . . 25

4.3.1 Harris Corner Detection . . . . . . . . . . . . . . 25

4.3 .2 Corner Pair Matching . . . . . . . . . . . . . . 26

4.3 .3 Searching Corner Pairs . . . . . . . . . . . . . . 27

5 Proposed Work . . . . . . . . . . . . . . . . . . . . . . . . . 28

5.1 Preprocessing . . . . . . . . . . . . . . . . . . . . . . . . . . . . . 29

5.2 Skeleton Classification . . . . . . . . . . . . . . . . . . . 29

5.2 .1 Branch Segments . . . . . . . . . . . . . . . . 29

5.2 .2 Intersection Points . . . . . . . . . . . . . . . 30

5.2 .3 Classification Algorithm _. . . . . . . . . . . . . 30

5.3 Intersection Point Statistics $\ldots \ldots \ldots \ldots$

5.3 .1 Intersecting Branches . . . . . . . . . . . . . . 32

5.3 .2 Angle of Intersection . . . . . . . . . . . . . . . . 33

5.3.3 Angle Difference from Reference Angle . . . . . . . . . . . 34

5.3 .4 Adjusted Width . . . . . . . . . . . . . . . 35

5.4 Sucker Characteristics . . . . . . . . . . . . . . . . 38

5.4.1 Branch Segments of Interest . . . . . . . . . . . . . 38

5.4 .2 Search Branch Segments . . . . . . . . . . . . . . . 40

5.4 .3 Potential Sucker and Verification . . . . . . . . . . . 40

5.5 Identifying Suckers . . . . . . . . . . . . . . . . . . . . 42

5.5 .1 Recursive Kick Off $\ldots \ldots \ldots \ldots$

5.5 .2 Crawl Up . . . . . . . . . . . . . . . . . . . 43

5.6 Pruning Suckers . . . . . . . . . . . . . . . . . 45

5.6 .1 Identifying Sucker Pixels . . . . . . . . . . . . . . . 45

5.6 .2 Determining Endpoint _ . . . . . . . . . . . . 46 
5.6 .3 Recursive Prune. . . . . . . . . . . . . . . . . . . . . 47

6 Evaluation . . . . . . . . . . . . . . . . . . . . . . 50

6.1 Identifying Sucker Branches . . . . . . . . . . . . . 50

6.1.1 Original Test Dataset . . . . . . . . . . . . . . . . 50

6.1.2 Downsampled Test Dataset . . . . . . . . . . . . . 66

6.2 Marking Endpoint of Sucker . . . . . . . . . . . . . . . . 69

6.3 Timing . . . . . . . . . . . . . . . . . 70

7 Conclusions . . . . . . . . . . . . . . . . . . . . . 72

7.1 Algorithm Effectiveness . . . . . . . . . . . . . . . . . . . . 72

7.2 2-Dimensional Images . . . . . . . . . . . . . . . . . . . . 72

7.3 Processing Speed . . . . . . . . . . . . . . . . . 73

7.4 Skeleton Classification . . . . . . . . . . . . . . . . . . 73

8 Future Work . . . . . . . . . . . . . . . . . . . . . . . . . 74

8.1 Improved Endpoint Marking . . . . . . . . . . . . . . . . 74

8.2 3-Dimensional Point Cloud . . . . . . . . . . . . . . . . . . . 74

8.3 Filtering Background Pixels _ . . . . . . . . . . . . . 75

8.4 Non-Heuristic Approach for sucker characteristics . . . . . . . . 75

8.5 Full Scale Autonomous Pruning System . . . . . . . . . . . . . 75

8.5.1 Greenhouse Preparations . . . . . . . . . . . . . . . . 75

8.5 .2 Algorithms . . . . . . . . . . . . . . . . . 76

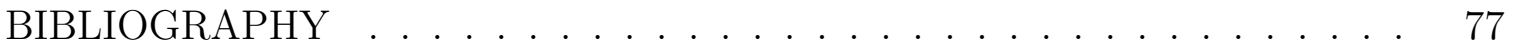
APPENDICES

A Appendix A ....................... 80

A.1 Downsample . . . . . . . . . . . . . . . . . . . . . 80

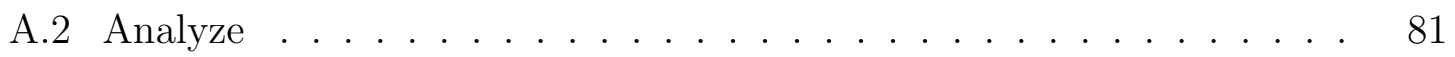

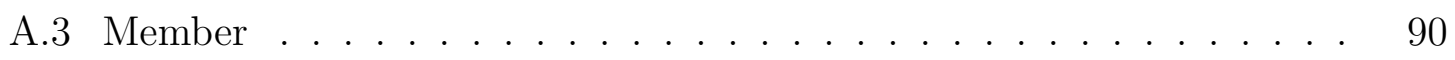

A.4 Branch . . . . . . . . . . . . . . . . . . . . . . . . 93

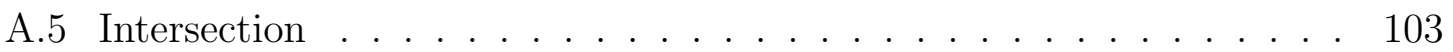




\section{LIST OF TABLES}

Table

6.1 Accuracy for the 100 original images . . . . . . . . . . . 51

6.2 Accuracy for the 873 downsampled images . . . . . . . . . . 68

6.3 Accuracy for the 97 unique images . . . . . . . . . . . . . . 68

6.4 Errors by downsampled rate . . . . . . . . . . . 69

6.5 Marking endpoint accuracy . . . . . . . . . . . 70

6.6 Timing for the 100 original test images . . . . . . . . . . . . . 71 


\section{LIST OF FIGURES}

Figure Page

1.1 Tomato sucker $[13] \ldots \ldots \ldots \ldots$. . . . . . . . . . . . 2

$2.1 \quad$ Determinate vs indeterminate $[2] \ldots \ldots \ldots$

2.2 Tomato suckers $[10] \ldots \ldots \ldots \ldots$. . . . . . . . . . 6

3.1 Grapevine structure $[12] \ldots \ldots$. . . . . . . . . . . 10

3.2 Selecting the pruning point $[12] \ldots \ldots$. . . . . . . . 13

3.3 (a) shows the original cluster and (b) shows the splitting of the two clusters to reduce error $[18]$. . . . . . . . . . . . . . . . . 18

4.1 Correlating skeleton . . . . . . . . . . . . . . 24

4.2 A corner pair (red) intersecting a sucker (blue) . . . . . . . . . . 27

5.1 Overview of proposed algorithm . . . . . . . . . . . . 28

5.2 Blue pixel represents the BS pixel, the green pixels represent neighboring skeleton pixels . . . . . . . . . . . . . . . . . 29

5.3 Blue pixel represents the IP pixel, the green pixels represent neighboring skeleton pixels . . . . . . . . . . . . . . . 30

$5.4 \quad$ IP statistics example . . . . . . . . . . . . . . . . . . . . . 32

5.5 Example of the outside-in approach superior to the inside-out . . . 34

5.6 Right and left BS with respect to a BSOI . . . . . . . . . . 35

5.7 Histogram comparisons between a uniform BS and a leaf BS . . . . 37

5.8 BS 2 represents a leaf, the white marks the uniform-like section of BS $2 \ldots \ldots . \ldots \ldots 7$

5.9 Overlaid image of the skeleton and the original . . . . . . . . 39

5.10 Example of potential sucker selection and verification . . . . . . 41

5.11 Four intersecting BSs with middle BS as the potential sucker . . . . 42

5.12 Comparison of the sucker marked with and without endpoint . . . . 46

5.13 Example of a complex sucker where recursive pruning is needed . . 47

6.1 Both suckers missed due to orientation of image . . . . . . . . 52

6.2 One of the suckers is missed due to failure of sucker characteristic . 53

6.3 Missed BSOI resulted in a missed sucker . . . . . . . . . . . 54

6.4 Multiple suckers spawning from same point . . . . . . . . . 55 
6.5 Multiple suckers spawning from same point . . . . . . . 56

6.6 Multiple suckers spawning from same point . . . . . . . . 56

6.7 Multiple suckers spawning from same point . . . . . . . . 57

6.8 Two suckers identified in an image . . . . . . . . . . . 57

6.9 Two suckers identified in an image . . . . . . . . . . 58

6.10 Complex plant structure . . . . . . . . . . . . . . . . . . 59

6.11 Complex plant structure . . . . . . . . . . . . . . . . . 59

6.12 Complex plant structure with leaf sucker . . . . . . . . . . 60

6.13 Complex plant structure with leaf sucker . . . . . . . . . . . . 60

6.14 Wide sucker . . . . . . . . . . . . . . . . . . . . . 61

6.15 Wide sucker . . . . . . . . . . . . . . . . . . . 61

6.16 Wide sucker . . . . . . . . . . . . . . . . . . . 62

6.17 Wide sucker . . . . . . . . . . . . . . . . . . . . . . 62

6.18 Leaf sucker . . . . . . . . . . . . . . . . . . . . 63

6.19 Leaf sucker . . . . . . . . . . . . . . . . . . . 63

6.20 Leaf sucker with complex plant structure . . . . . . . . . . . . . 64

6.21 Leaf sucker . . . . . . . . . . . . . . . . . . . . . . . 64

6.22 Thin sucker . . . . . . . . . . . . . . . . . . 65

6.23 Thin sucker . . . . . . . . . . . . . . . . . 65

6.24 Thin sucker . . . . . . . . . . . . . . . . . . . . . 66

6.25 Skeletonization error: visible loop not representative of the structure 67

6.26 Both cases of marking failure . . . . . . . . . . . 70 


\section{Chapter 1}

\section{INTRODUCTION}

Indeterminate or vining tomatoes are the most popular types of tomatoes in high tunnel and greenhouse mass production. Indeterminate tomato plants can grow and produce fruit in the greenhouse environment continuously, allowing growers to engage in an uninterrupted harvest throughout the growing seasons. In order to sustain a high level of productivity, tomato plants must be routinely pruned to ensure nutrients are delivered to the main stem and fruit, rather than wasted on extraneous growths, commonly referred to as suckers (shown in Figure 1)[9]. Routinely pruned tomato plants can direct their energy towards creating and ripening fruit instead of more leaves. The fruit derived from adequately pruned tomato plants has also been found to be of a higher quality than fruit acquired from unpruned plants. Previous research has shown that tomato plants whose stems underwent pruning had about 40 to 50 percent higher marketable yield $[14,7]$. In contrast, while unpruned plants did yield more fruit due to the increased number of branches and the resulting increased flowering surface, fruit from the plants was smaller and unmarketable in many cases. The size of the tomato was found to be inversely related to the number of stems allowed to grow $[16]$.

Additionally, well-pruned tomato plants benefit from improved airflow. This makes them less susceptible to disease and pest attacks; two issues plants with a thick canopy and wet environment often face. [14]. Wet environments allow for the rapid growth of bacteria while thick canopies make it difficult for growers to spot and exterminate pests. Moreover, with fewer fruits to be nourished, fruit ripens faster on a pruned plant. This and the aforementioned benefits regarding higher quality yields and durability are all reasons as to why the pruning of tomato plants is an integral 
process for efficient growing.

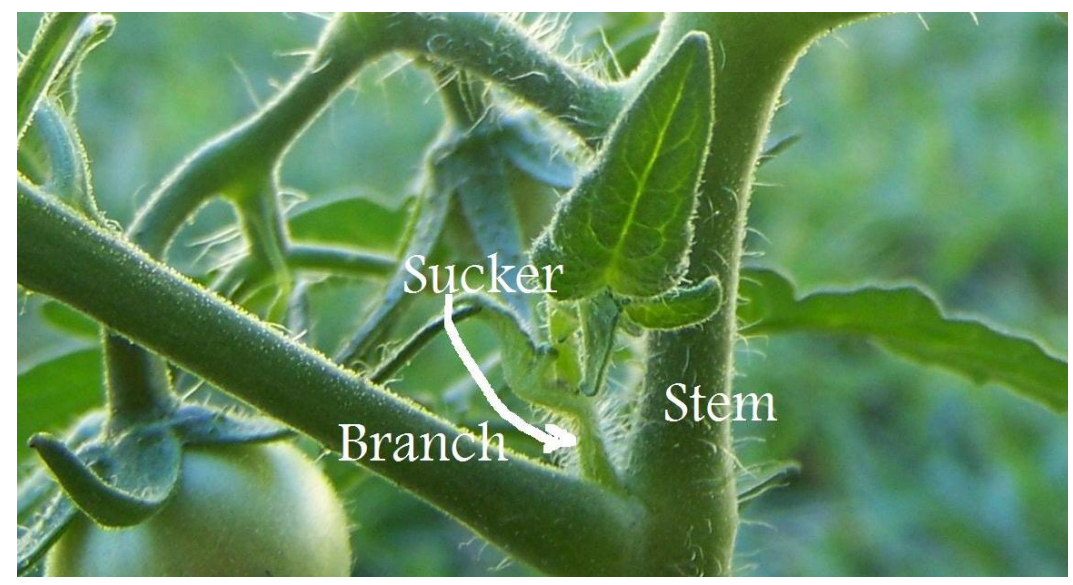

Figure 1.1: Tomato sucker [13]

In current practice, the pruning of indeterminate tomato plants requires extensive labor, and it is a completely manual process. This manual pruning process relies heavily on knowledge of tomato plant pruning and labor in order to have higher yields of marketable fruit and overall quality [8]. Tomato growers in California suffer from an insufficient number of farm laborers and increasing labor costs. This seems to be an issue that will persist over time regardless of future changes in immigration laws. Because the process of tomato pruning is still manual, workers must inspect and prune each tomato plant within a greenhouse. The answer to a more efficient, accurate, and cost-effective solution lies in an automated system that utilizes computer vision algorithms and automation equipment to prune tomato vines. This solution would hopefully improve the profitability of farmers, reduce the cost of labor, and improve the health and quality of the tomato plants being harvested.

This project proposes a novel algorithm for detecting suckers without depth sensors. Our research aims to be the first step towards a fully autonomous system for pruning tomato plants. Considerable advancements in computer vision have enabled many agricultural applications. Many researchers have tackled pruning of orchards 
and grapevines, but automated pruning of tomatoes has yet to be investigated. By building upon existing research and innovations, this project aims to reduce the manual requirements for pruning indeterminate tomato plants, lower labor costs, and increase farmers' profitability and marketable crop yields. 
Chapter 2

\section{BACKGROUND}

Important information about indeterminate tomatoes and the technologies used in this thesis are discussed in this chapter.

\subsection{Greenhouse Indeterminate Tomatoes}

This section covers the physical structure and definition of greenhouse indeterminate tomatoes, and the pruning points for these plants.

\subsubsection{Definition}

Indeterminate tomato plants vary from determinate tomatoes in many ways, but the most notable is the continual growth of indeterminate tomatoes. Indeterminate tomato plants, however, can live for multiple seasons as long as they do not succumb to the cold weather. This longer lifespan allows for a more continuous supply of fruit, as opposed to one large harvest from determinate plants. It also promotes growing in greenhouses which permits flexibility of growing locations. Determinate plants typically bear fruit and die off within a couple weeks. These plants are often not pruned because their lifespan is so short. Figure 2.1 shows a visual difference between the two types of tomato plants. The determinate plant is much shorter and bushier than the indeterminate counterpart. 


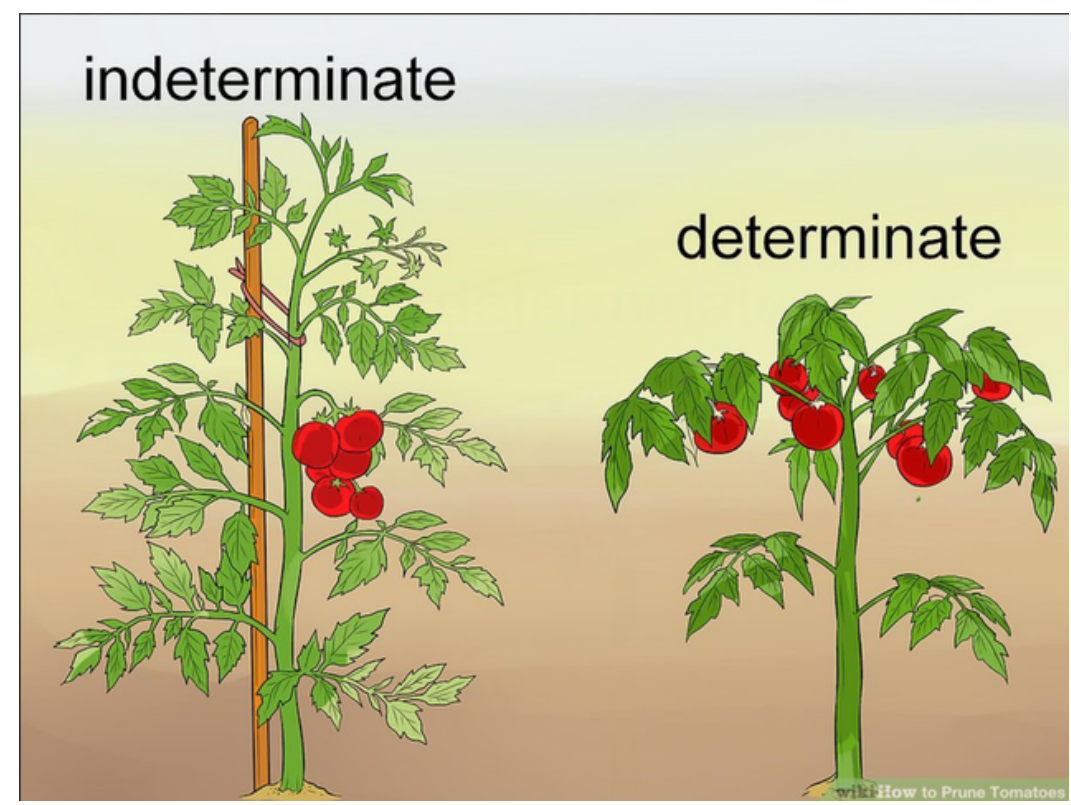

Figure 2.1: Determinate vs indeterminate [2]

\subsubsection{Pruning Points}

The continuous growth of indeterminate tomatoes comes at a cost of constantly having to prune and maintain the plants within the greenhouse. The pruning points are often referred to as suckers. These are defined as small shoots that bisect the main stem and a supporting branch. If left unpruned, it is possible for the sucker to become thicker and larger than the supporting branch or main stem. Given this fact, a tomato sucker is defined as any shoot or branch that bisects the main stem and another branch. 


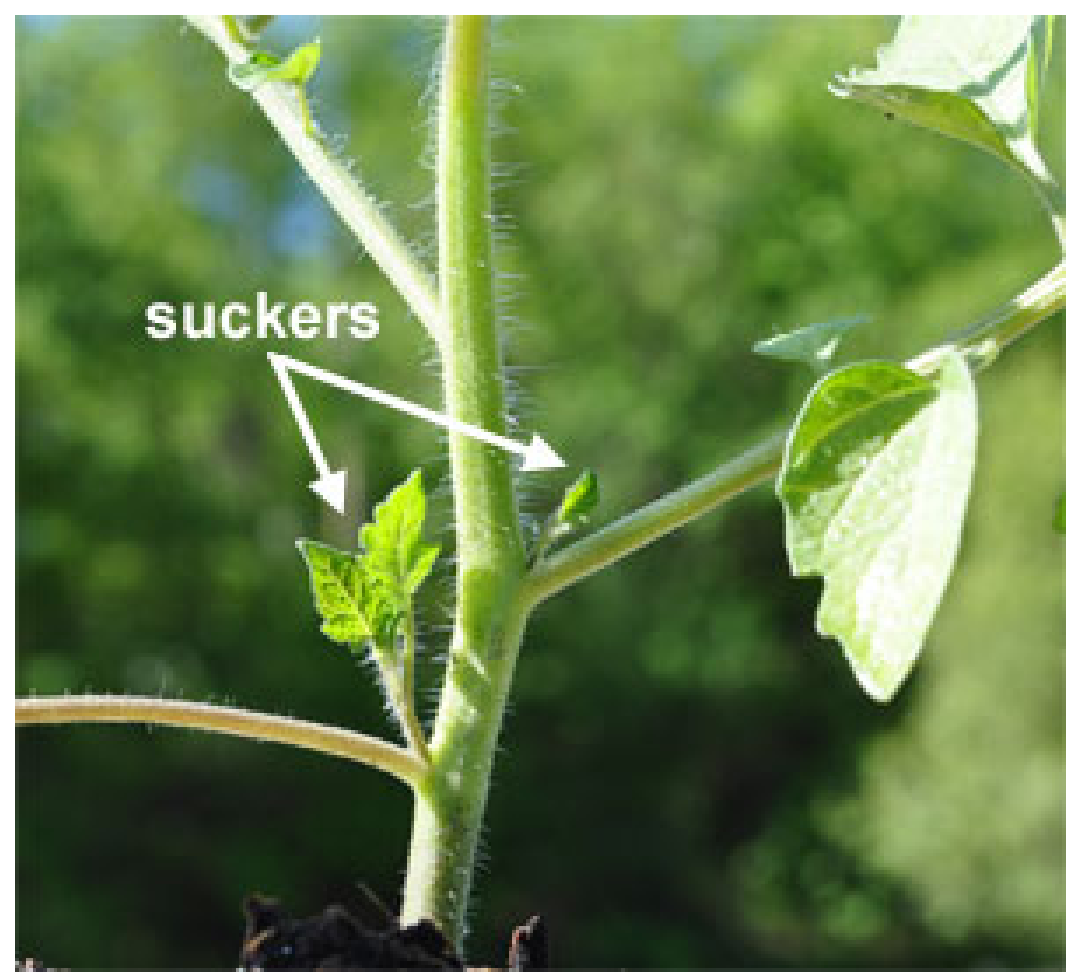

Figure 2.2: Tomato suckers [10]

\subsection{Leveraged Technologies}

All software for this thesis was written in python for multiple reasons: its popularity, ease of use, and widely available open sourced packages. The following open source python packages were utilized in this project's work.

\subsubsection{OpenCV}

OpenCV is an open source computer vision library, mainly for real-time applications. The library is written in $\mathrm{C}++$, but it includes APIs for other languages such as Python, Java, and Matlab. It contains functions and algorithms for computer vision and machine learning applications including implementations of thresholding, clustering, and classification algorithms [4]. The primary functions leveraged 
from OpenCV include reading and writing images, grayscaling, thresholding, and the connectedComponents function.

\subsubsection{NumPy}

Numpy provides powerful N-dimensional arrays for performing quick and sophisticated calculations. It offers abilities to quickly modify and index into multi-dimensional arrays, making it a perfect tool for computer vision applications [3]

\subsubsection{SciKit}

Scikit-image is a collection of algorithms for image processing. The medial axis algorithm is used for the skeletonization aspect of the algorithm developed in this thesis [5].

\subsection{Depth}

All test images were taken with a $29 \mathrm{~mm}$ focal length, $12 \mathrm{MP}$, digital camera without any depth capabilities and some assumptions were made because of this. Without depth sensors, it is very difficult to filter background pixels, especially in a cluttered environment, such as a tomato plant. For this reason, all test images were manually cropped to exclude the background. Again, without depth sensors, the third dimension in an image is very difficult to insinuate from only a 2-dimensional image. For this reason, all test images were assumed in a 2-dimensional plane.

\subsection{Scope}

The scope of this thesis is to intake a closeup image of a tomato plant's stem with the background excluded and output the same image with only the suckers marked red. 
The sucker detection algorithm developed in this thesis does not take into account depth in an image. 
Chapter 3

\section{RELATED WORK}

There has been past work done with automated pruning of different plants and trees. Several notable efforts were reviewed and reflected in this section. Some of the ideas presented in these past works were investigated for their relevance to this thesis.

\subsection{Grapevine Pruning}

Throughout the years, automation of tasks and operations on vineyards have been developed to save on labor costs. One of the tasks that still remains a manual process is pruning vineyards. Pruning vineyards plays a crucial role in the quality of fruit and yield of the harvest. The pruning process involves very precise cuts which is not possible with any current robotic system. Identifying the nodes or buds, is one of the key components of identifying the pruning points for grapevines $[19,12]$. Once the buds are detected, various pruning methods can be applied, such as the two bud spurring method. Two bud spurring is a pruning technique that involves cutting the top cane and cutting last season's canes back to two to three buds [12]. The structure of a grapevine and where to prune is shown in Figure 3.1. Grapevines are often supported with canopy wires running horizontally. These canopy wires must be detected for a robotic system to accurately prune the grapevines without damaging the canopy structure. If a wire is inadvertently cut or collided with, the repair time can take hours and the grapevines will likely be damaged [17]. 


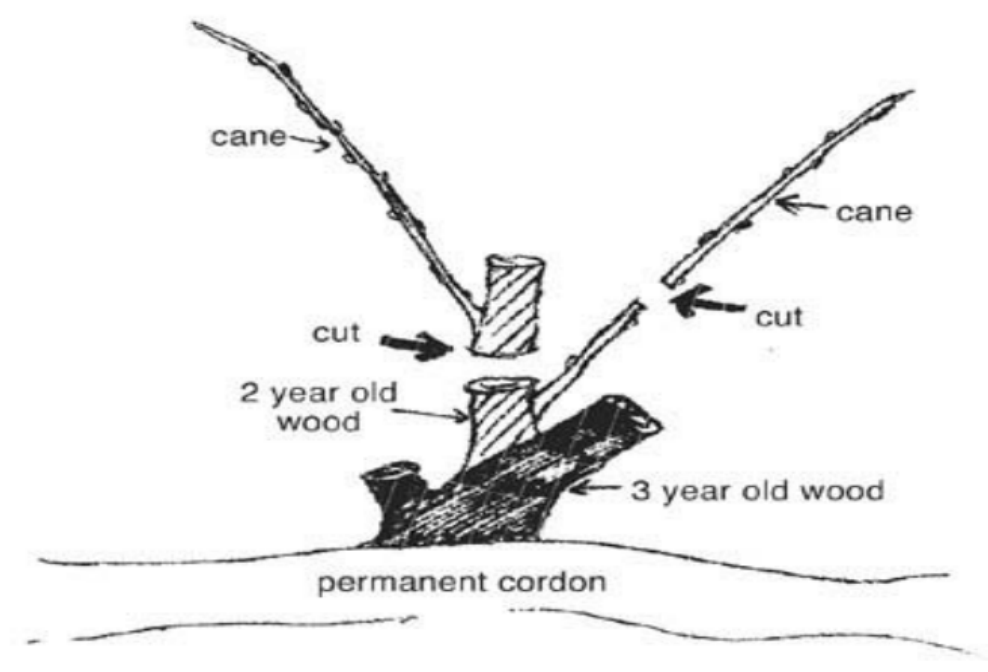

Figure 3.1: Grapevine structure [12]

\subsubsection{Image Preprocessing}

With every computer vision application, image preprocessing is performed on images to highlight pixels of interest and exclude everything else. Like most agricultural plants, grapevines are grown in rows. They also typically grow in two dimensions along guided, horizontally placed wires. These presumptions of grapevines allow the background of grapevines to be eliminated with the use of a white material placed behind them. Thus, the background is assumed to be white and act as constant illumination to focus the algorithm on the image analysis component $[12,19,17]$. This eliminates much of the preprocessing required and simplifies the overarching algorithm. To reduce noise in the image, the weighted mean filtering method is used and a threshold is chosen to obtain a two state image. But the two state image contains disjointed grapevines. To solve this problem, the researchers applied the closing morphological operation [19]. This involves dilating the holes in the image. Due to the grapevines' relatively uniform color, the three components in a color 
image offer little benefit when determining the pruning points. For this reason, the image is gray-scaled to eliminate the overhead of three color components, and reduce the overall image size by $66 \%[12,19]$. The three color components, RGB, were investigated to determine which one exhibited the most clear difference in background pixels and grapevine pixels. The B component exhibited the largest difference, so it was used as the component to grayscale the image [19].

\subsubsection{Identifying Canes}

As shown in Figure 3.1, the canes are the small branch runners spurring from the thick wood sections of the grapevines. The preprocessing step described in subsection 3.1.1 yields a binary image where black pixels are part of the grapevine and white pixels are the background. The locating of the canes was done trivially by defining a starting search point to be $1 / 3$ the height of the original image [12]. From here a cane was identified as the first black pixel on the horizontal plane. Once a black pixel is encountered, the midpoint of the cane is determined by traversing the horizontal plane until a white pixel is encountered. This process is repeated in the vertical axis to obtain midpoints for the entire cane. To address crossing canes, the researchers used Linear Least Squares Error Fit to obtain an approximate short line. This determines the expected slope of the cane. In the event of canes crossing, the approximation of the cane was used to determine which pixels were relevant to the current cane. After this method, the midpoints and width of each cane were identified. Using this process, $98 \%$ of canes were detected in the 10 sample images used [12].

\subsubsection{Identifying Buds}

Buds exhibit three distinct physical features: nodes on the canes are visibly thicker in the horizontal axis than the cane, the slopes of the cane above and below the bud 
differ, and the grayscale changes when a bud is present $[12,19]$.

Gao et al. only used the width difference to determine the nodes on a cane. The process in determining the width of the canes was used to identify the nodes. If the width varied by a certain threshold, then a node was marked at the position of interest [12].

To counter the fact that width of the branches vary, Xu et al. decided to leverage corner detection on a skeletonized image to detect the buds. The researchers chose a parallel thinning algorithm for the morphological step, due to its proven superiority over its serial counterparts. The Rosenfield thinning algorithm outperformed the Zhang thinning algorithm because it more accurately maintained the shape features of the grapevines. The next step was to run corner detection on the skeletonized image. The researchers chose to use the Harris operator because of its history of reliability and efficiency. This method yielded the detection of real and pseudo buds. Pseudo buds are defined as the point at which two canes intersect. The researchers were able to positively identify $76.5 \%$ of the buds with an error rate of $17.8 \%$ in 115 sample images. Since grapevines are rough in nature, the corner detection algorithm would often falsely identify a bud. Also, the thinning algorithm would sometimes smooth out the buds, rendering them undetectable by corner detection [19].

\subsubsection{Identifying Pruning Points}

The pruning point lies somewhere between the second and third node on a cane. Once all the nodes are identified on a cane, the algorithm counts two nodes up from the base of the cane and picks and marks the middle point between the second and third node. The pruning process is visualized in Figure 3.2. 


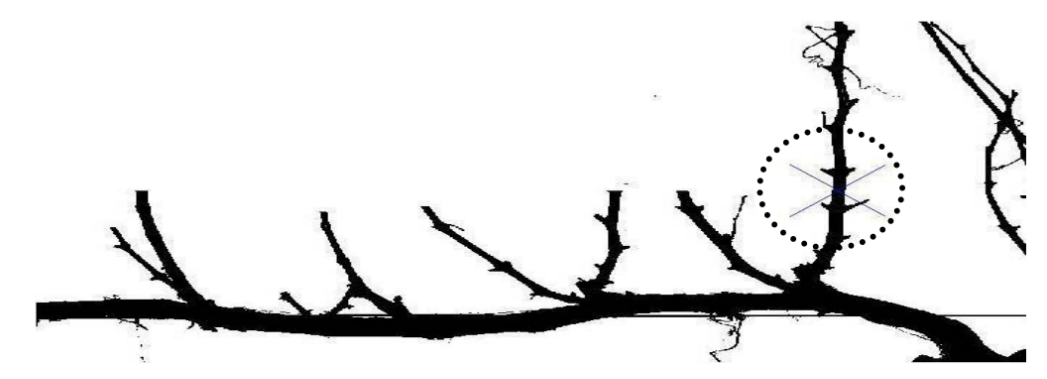

Figure 3.2: Selecting the pruning point [12]

\subsubsection{Identifying the Canopy}

Previous work has attempted to identify wires using a heuristics approach, but that approach requires fine tuning by a programmer to achieve acceptable results under different conditions such as lighting. Researchers at University of Canterbury propose a system that leverages neural networks with a feedback loop in order to adapt to changing conditions.

Classifiers were generated by using a 9x9 square region centered around the pixel of interest. This two dimensional grid was flattened into a vector to be used by the neural network. All images were converted to bayer images. A bayer image consists of 8 bit numbers that represent the intensity of a color component: $R, G$, or B. To obtain a full RGB image, a bayer filter is applied across the bayer image. Each pixel's RGB value is determined by the neighboring intensity values in the bayer image.

Neural networks require both positive and negative samples for training. A single sample image with manually marked canopy wires was used for training. 1,000 positive and 10,000 negative samples were randomly selected from the sample image. The researchers determined more negative samples yielded better results, since wires and grapevines are structurally similar. Since pixels in bayer images can represent a different color depending on its location, four neural networks were trained to take this into account. 
Once the neural network was trained, the vector representation of a pixel was fed into the neural network and a simple threshold was used to determine whether the pixel was a canopy wire or not. The output of the neural network for an image is written to a separate image, yielding a binary image.

To reduce noise, a simple 5x3 region centered around the pixel of interest was used. The reasoning behind having more pixels in the horizontal direction of a region is that wires typically run horizontally. If eight or more pixels within the region were wire pixels, than the pixel of interest was determined to be a wire pixel. The researchers tested with various thresholds to determine if the pixel was indeed a wire pixel and found eight to be the best number.

A single 960 x 1280 pixel image was used to test the above steps to classify canopy wire pixels. The researchers obtained a precision of $94.3 \%$ using the above steps and the image took a total of 27.1 seconds to process.

The implementation used by the researchers operated on a single thread. Parallelizing the algorithm will greatly improve the speed and performance of classification. Rather than a single training image, more sample images can be used by the neural network to improve accuracy and precision [17].

\subsection{Orchard Pruning}

Dormant tree pruning is vital to the next seasons yield and quality of fruit. Pruning remains one of the most costly and labor intensive operations in specialty crop production. Pruning of dormant trees involves cutting primary branches or PBs (branches that connect directly to the trunk of the tree). Parameters such as diameter and angle between trunk and PB are used to determine pruning points. To obtain these parameters, a 3D representation of the tree is necessary to accurately choose the pruning points $[11,18,6]$. 


\subsubsection{Data Acquisition}

Three popular choices for generating a 3D model are stereo vision cameras, the kinect sensor, and laser options. Stereo vision cameras rely on constant illumination and other hard-to-control variables [18]. The kinect sensor is relatively cheap and accessible, but accuracy and reliability is questionable $[11,6]$. The laser options are very expensive when compared to the other two data acquisition options, but their accuracy tops all three $[11,18]$.

Elfiky and Akbhar et al. opted to utilize the Kinect sensor along with the KinectFusion (KF) software. The Kinect Sensor provides an affordable option that is capable of providing depth images at a relatively high frame rate of 30 frames per second. The Kinect Fusion software is accurate at generating 3D models of objects if one can walk around the object and acquire continuous, overlapping photos. But this is not the case for orchard trees. Typically, only images of the front and the back of the tree are accessible. Their research into 3D modeling of dormant trees doubles as a case study for the accuracy of the Kinect sensor $[11,6]$.

Medeiros et al. chose to use the more accurate laser option to easily filter out background noise common in orchards. The specific laser used was the SICK LMS111 mounted on an Aerotech ART310 rotary stage. The ART310 was then mounted on an ATS02060 linear slide. The entire system was mounted on an N.BLOSI apple picking platform. This enabled movement of the laser in the $\mathrm{x}, \mathrm{y}$, and $\mathrm{z}$ direction to obtain full coverage of the tree in question. For each tree analyzed, a total of 40 images were taken; 10 at 1.5 meters high, and 10 at 4 meters high varying the degree of the laser by $0.45^{\circ}$ each image on each side of the tree [18]. 


\subsubsection{Obtaining 3D Point Cloud}

A $3 \mathrm{D}$ point cloud is necessary for correctly measuring the diameter of the primary branches and trunk.

One method for the reconstruction involves taking a picture of the front and back of the tree with a Kinect Sensor. Each depth image is then fed into the Kinect Fusion software to obtain a 3D point cloud. The Laplacian Smoothing function is run on the entire point cloud to obtain a skeleton. Once the skeleton is acquired, geometric features are extracted from each image, and the results are calculated to determine where to merge the two point clouds. The geometric features are defined as the trunk and primary branch nodes. Once the trunk and primary branch nodes are determined, three parameters are utilized to calculate the correspondences between the front and back point clouds: the angle between the secondary branches and the connected primary branches, the distance between the primary branches and root of the tree, and the distance between the primary branch and the trunk. These three feature points for each primary branch is used along with the Iterative Closest Point (ICP) algorithm to mesh the two point clouds together [11].

A simpler method that only involves one picture uses a conversion equation to obtain a point cloud. A depth image is obtained with a Kinect Sensor and converted to a point cloud using the camera intrinsic matrix and the depth value for each pixel $[6]$.

Medeiros et al. used 40 different images to construct a single point cloud of a tree. Triangulation of each image involved applying a simple floating pixel removal filter to remove points with no neighbors and Laplacian surface smoothing to reduce noise. The 40 separate point clouds were combined using the iterative closest point algorithm [18]. 


\subsubsection{Trunk Localization}

Trunk nodes are obtained by starting at the bottom-most node of the skeleton image, moving up the $\mathrm{z}$ axis, and checking the connectivity matrix to determine if it is part of the trunk. Any nodes that are not a member of the trunk, are considered branch nodes and a subsequent algorithm is used to segment the nodes of the point cloud into primary branches [11].

Rather than a node by node approach, clustering can be done on the image to cluster nearby nodes before the localization step [18]. A split and merge algorithm that utilizes the Kmeans clustering algorithm results in a decently partitioned point cloud where clusters are approximated with cylinders.

First, a clustering algorithm runs Kmeans to obtain a general clustering of the nodes. Second, the split step is operated on each cluster. The split involves fitting a cylinder to the nodes within the cluster using the least squares approach. If the error is greater than a certain threshold, the cluster is split again using Kmeans. The split step is shown in detail in figure Figure 3.3. Third, the merge step is performed on all clusters to mitigate clusters that have been partitioned too much. The euclidian distance between each cluster's fitted cylinder's center is calculated and if the distance is less than a certain threshold, the two clusters are merged [18]. 


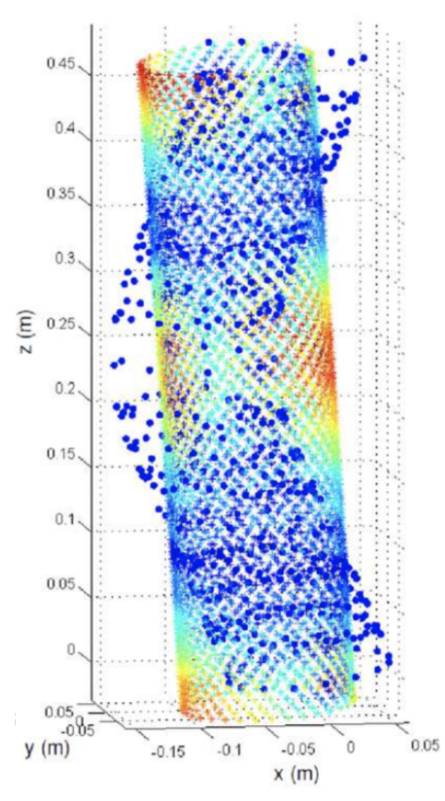

(a)

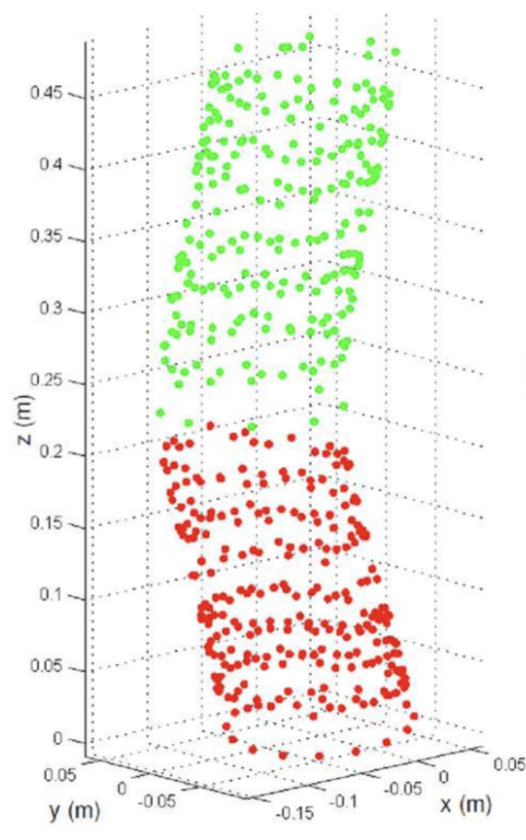

(b)

Figure 3.3: (a) shows the original cluster and (b) shows the splitting of the two clusters to reduce error [18]

Once the clustering is complete, the cluster with the smallest $\mathrm{z}$ value is used as the basis for the trunk and the starting point for the trunk localization algorithm. Clusters are defined to be connected if they share an equal number of closest neighbors with one another. Furthermore, clusters are defined to be apart of the trunk if they are connected with an existing trunk cluster within a certain thresholded angle. Using this definition, the algorithm moves up the $\mathrm{z}$ axis, grouping the clusters in such a way that all trunk nodes in the point cloud are identified [18].

\subsubsection{D Model fitting to 3D Point Cloud}

Elfiky et al. modeled the tree by fitting semi-circles to the cross sections of the point cloud. With a full scan of the tree, entire cylinders can be fitted around portions of the point cloud to obtain a 3D model [18]. 


\subsubsection{Diameter Measurement}

From the fitted semicircles, the diameter of any point along the skeleton can be measured within an error of $5 \mathrm{~mm}$ by looking at the diameter of the semi circle correlating with that skeleton point [11]. If a full 3D-model is generated, a more accurate diameter can be measured from the fitted cylinder [18].

\subsection{Relevance}

Tomato vines are very different from grapevines and trees in almost every aspect. Tomato vines are mostly green, but can sometimes be different colors. In our research, some of the younger tomato suckers exhibited black, purple, or even white color. Since tomato suckers can be identified at any stage of its growth, color is something our algorithm did not rely on. For the most part, grapevines exhibit a very uniform shape. However, tomato plants are bushy and have unpredictable growth patterns. Grapevines and orchard trees are fully visible in the dormant season, but greenhouse tomatoes never have a dormant season and thus the plant is fully shrouded with foliage. This makes a $3 \mathrm{~d}$ model of the entire plant difficult to obtain.

Despite the major differences between tomato plants, grapevines, and orchard trees, many of the applicable methods discussed in these past works were investigated and tested. Corner detection, that was used to identify grapevine buds, was tested and determined to be not that useful for detecting the corner edges of suckers. Kmeans clustering, used for clustering 3D points into branches of orchard trees, was attempted at clustering the tomato branches, but a faster and more reliable approach was discovered in this thesis.

The skeletonization method to obtain a simpler image of grapevines was found to be extremely helpful in simplyfying complex tomato plant images. Skeletonization 
became one of the key components in the algorithm developed in this thesis to detect suckers. 
Chapter 4

\section{INITIAL WORK}

This chapter aims to provide insight into the various methods experimented with before finalizing the implementation used in this thesis. These methods are not included in the final implementation of detecting suckers. However, they provide insight into the unsuccessful methods and reasoning as to why they did not work. The main topics covered in this chapter include methods for skeleton classification, finding and labeling the main stem, joining branch segments, and detecting sucker features.

\subsection{Skeleton Classification}

The skeleton, or morphological-thinned, image provides an easier object to analyze than a full image. The idea is to obtain a skeleton of the plant and use the skeleton to quickly read, analyze, and access each branch on the plant. The process to obtain the skeleton is discussed in the Proposed Work section. To analyze the skeleton, the branch segments (BS) and Intersection Points (IP) have to be found and labeled on the skeleton. This process is defined as skeleton classification.

\subsubsection{Hough Line Transform}

Hough Line Transform was the main component in the first method for classifying the skeleton. Hough Line transform is run on the skeleton and lines are merged that intersect one another at a thresholded angle. Hough Line requires multiple parameters such as $\rho, \vartheta$, accumeulatorThresh. The goal is for the sucker identification algorithm to be scale invariant and the parameters required for Hough Line would need to be 
altered depending on the image size, complexity, etc. Nonetheless, Hough Line was investigated and used in an attempt to classify the skeleton.

First, Hough Line was run on the skeleton image. The lines were returned and subsequently parsed. Each line was analyzed, checked for the distance between every other line. If the lines were within a specified thresholded distance and the difference between their angles were within an angle range, the lines were considered to be part of a branch segment. The results were subpar for a simple image. The skeleton was not classified well enough to be of any use.

\subsubsection{Kmeans Clustering}

In section 3.2, the pixels were clustered using a split and merge approach that utilized kmeans clustering. The pixels were split until the cluster was able to be fitted with a cylinder. Since that approach included 3D pixels, a cylinder seemed appropriate for determining if the cluster was sufficient. For a 2D image and a 1 pixel-wide skeleton image, a line of best fit seems more appropriate. The split and merge approach for classifying branches in a skeleton image proved to be too slow and the results were mediocre.

\subsubsection{Direct Neighbor Approach}

Originally, every pixel in the skeleton was going to be analyzed to see if the pixel should be labeled a BS or IP pixel. The first algorithm for labeling pixels is covered in Algorithm 1. Each pixel in the image was looped through and the number of direct neighbors of the pixel of interest determined if the pixel was part of a BS or an IP. If the pixel had 2 or less neighbors, it was determined to be part of a BS. If it had 3 or more neighbors, it was considered an IP. This method was sufficient for smaller images, but larger images, like the ones used in the test data set, were too 
slow. This method processed an average of 40,000 pixels per second. For a $2000 \mathrm{x}$ 4000 pixel image, it would take 200 seconds to label the skeleton pixels. For a real world application, the algorithm needs to be able to quickly analyze an image. For this reason, a faster method was developed and is discussed in subsection 5.2.3.

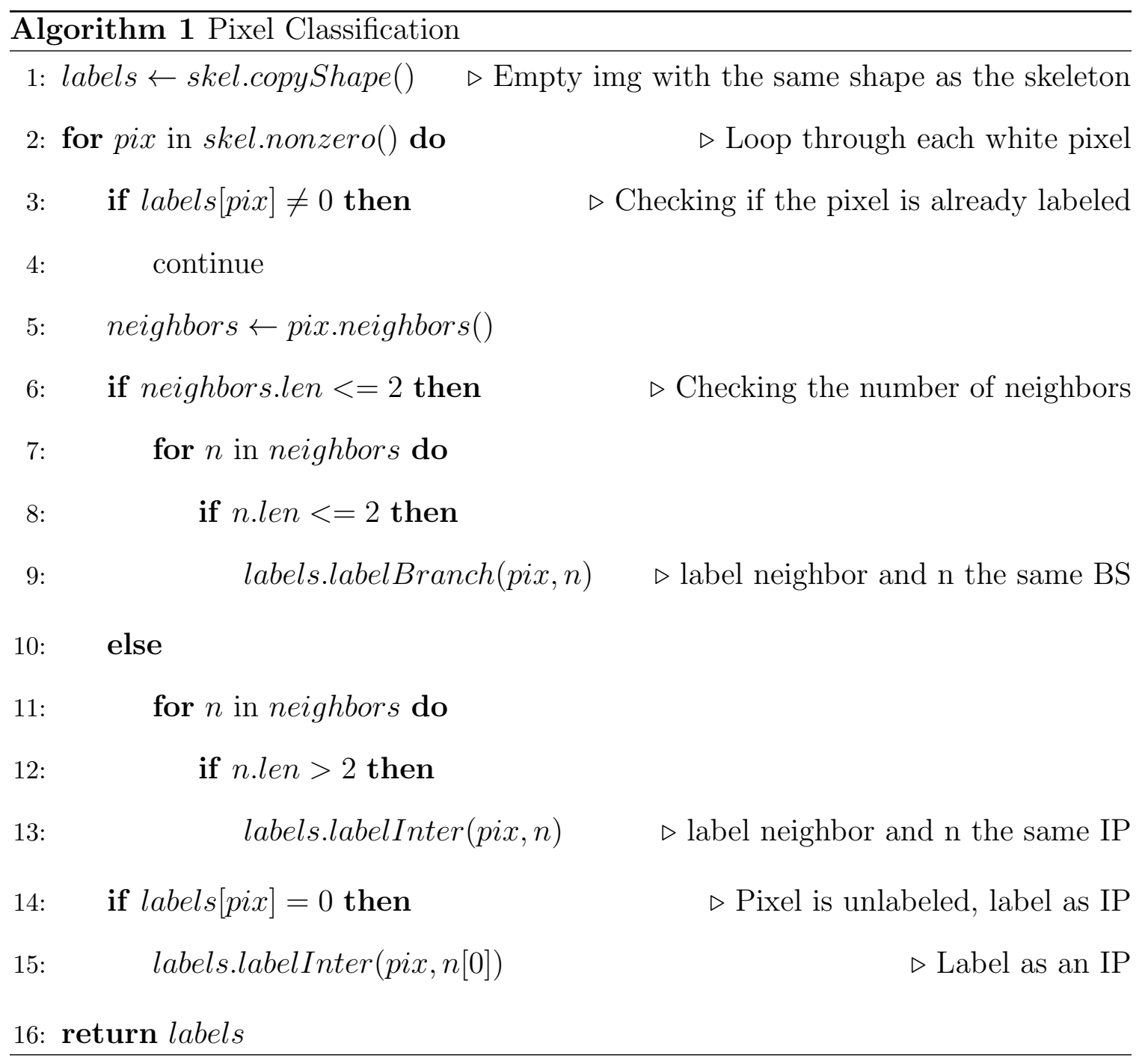

\subsubsection{Joining Branch Segments}

Labeling the pixels in the skeleton was the first step in classifying. The second step was to join BSs to obtain a more accurate representation. BS are terminated with an IP, but often times BS only represent a portion of the branch of the original image. 
This can be seen in Figure 4.1, BS 3, 6, and 7 all make up the left most branch in the image. The original algorithm for detecting suckers, discussed in section 4.3, relied on whole branches to be represented by a collection of BSs. BSs were joined by a similar method used in Algorithm 2. The correct BSs were merged for simple images such as the one in Figure 4.1, but more complex images yielded poor results with BSs being merged with incorrect counterparts.

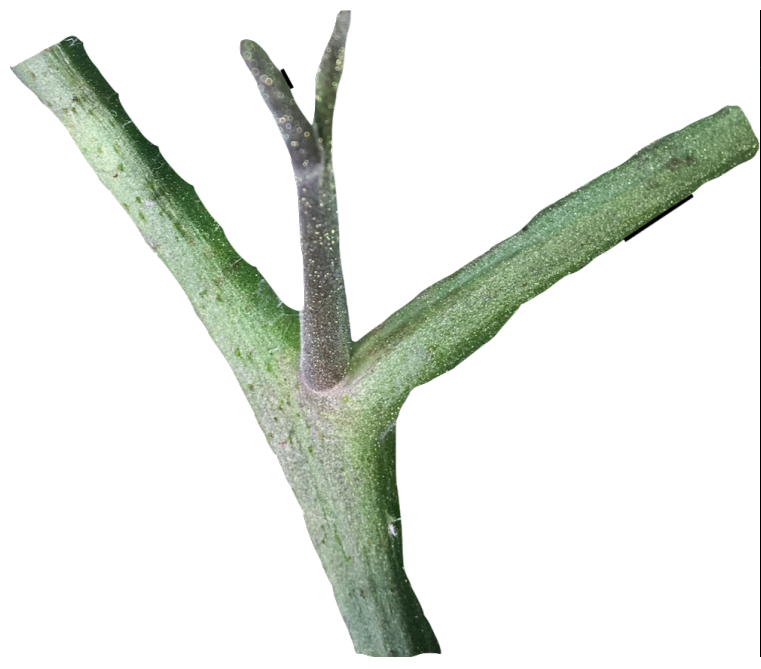

(a) Original image

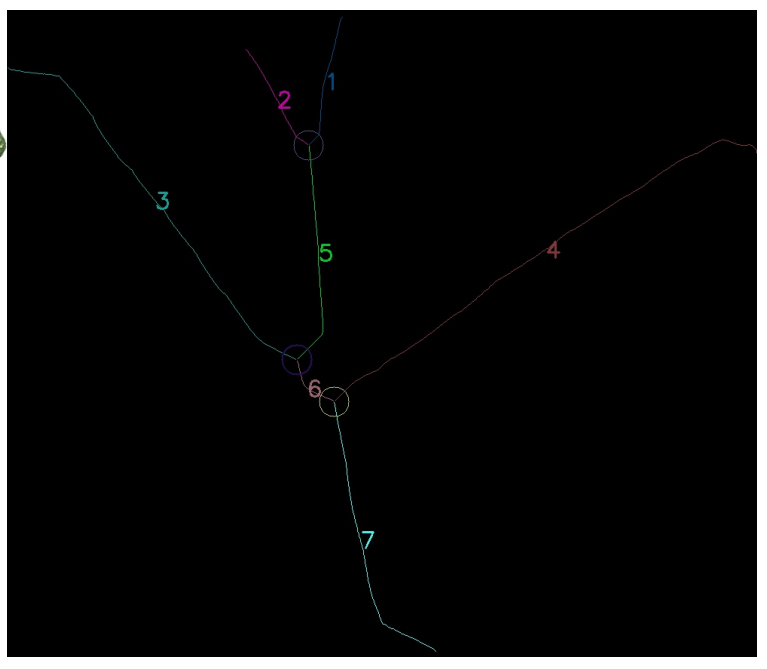

(b) Segmented skeleton

Figure 4.1: Correlating skeleton

\subsection{Finding and Identifying the Main Stem}

Once the classification of the skeleton was complete, the next step was to identify and label the main stem in the image. The main stem search algorithm started at the lowest BS and crawled up to the nearest IP. From there it picked the best BS to merge with the main stem using the width and angles of each intersecting BS. This was repeated until no more branches could be traversed. The main stem search algorithm is described in detail in Algorithm 2.

The first sucker detection algorithm relied mostly on knowing where the main stem was in the image. The main stem had to be identified accurately, and if it 
was not, the sucker was always either missed, or the wrong branch was inaccurately categorized as a sucker. The correct main stem was only identified about $50 \%$ of the time, causing our sucker detection rate to have a maximum accuracy of $50 \%$. Complex images with multiple branches and leaves almost never selected the correct main stem branches, further limiting the use case of this algorithm.

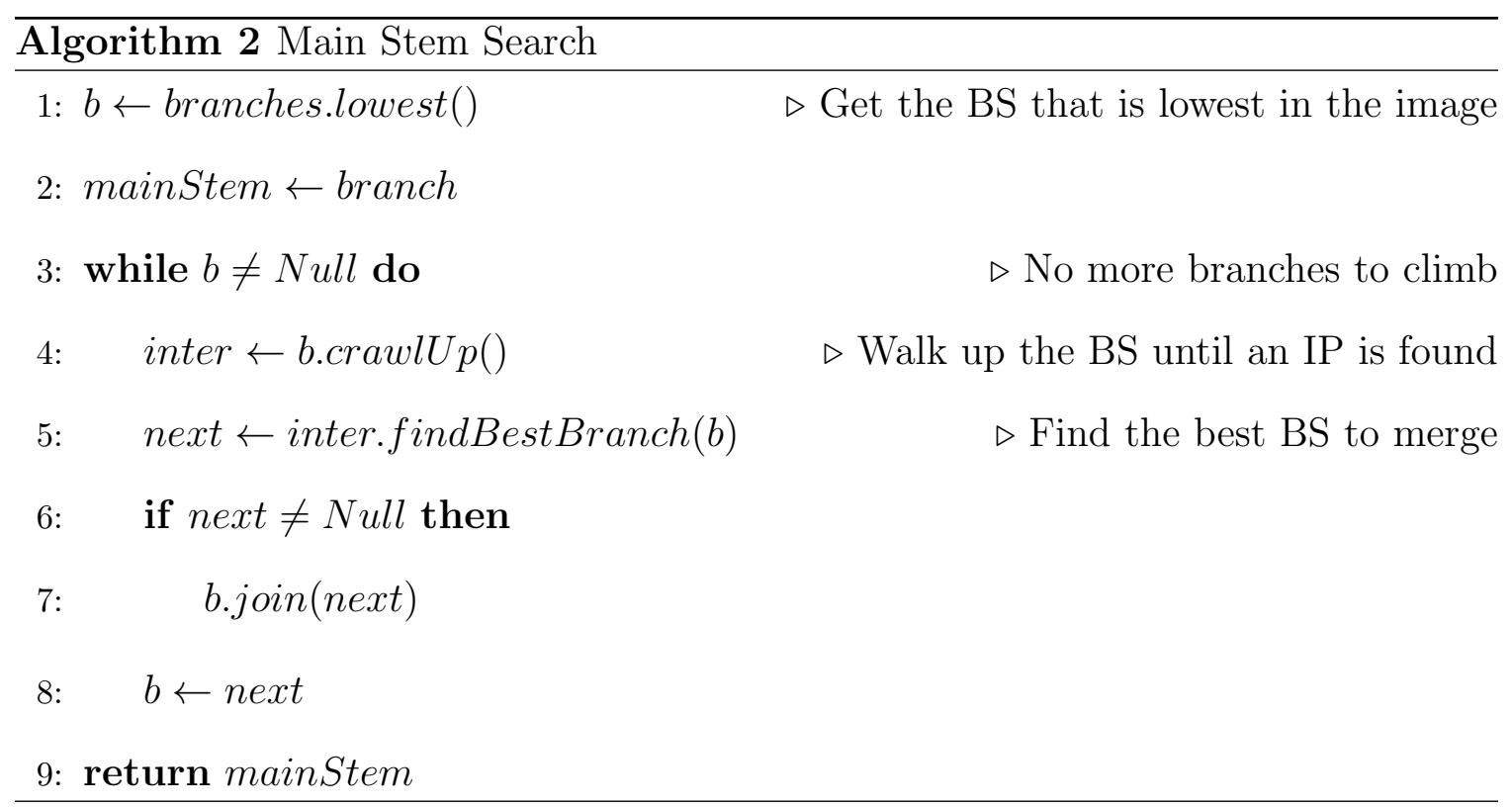

\subsection{Identifying Suckers}

The last step was to identify the suckers in the image. This includes running corner detection on the image to find points of sharp changes, pairing up the corner points to form valid corner point pairs, and finally searching each corner point pair to identify a sucker.

\subsubsection{Harris Corner Detection}

Harris corner detection required parameters much like the Hough Line transform, decreasing the robustness of this method. The block size of the neighborhood, aperture size of the Sobel derivative, and the Harris detector free parameter are all used when 
running the Harris corner detector. This corner detection algorithm proved to exhibit varying results on different images with different parameter values. OpenCV's Harris corner operator returns all the pixels in the image with an associated weight to them. This weight correlates with how strong the corner is. The higher the weight, the stronger the corner. Due to the nature of OpenCV's Harris corner detection, there were many steps that needed to be taken to make the data returned from the detector useful. First, the entire result had to be thresholded to filter out weak corners. The basis for filtering out weak corners was to filter out pixels that were not within a percentage of the maximum corner value. If the image contained a very strong corner, some of the weaker, but still relevant corners were filtered out from the image. To counter this, the threshold was set fairly low, which caused more corners to be parsed than necessary. Second, the centroids of the corner points had to be found, since a corner point actually consisted of multiple pixels clustered together rather than one single pixel.

\subsubsection{Corner Pair Matching}

The corner pairs were originally going to be the basis of a potential sucker. The idea was that each corner pair would intersect a BS, as shown in Figure 4.2. This was found to not be useful as corner pairs can have all sorts of arrangements and orientations which made the later subsection 4.3.3, Searching Corner Pairs, difficult. 


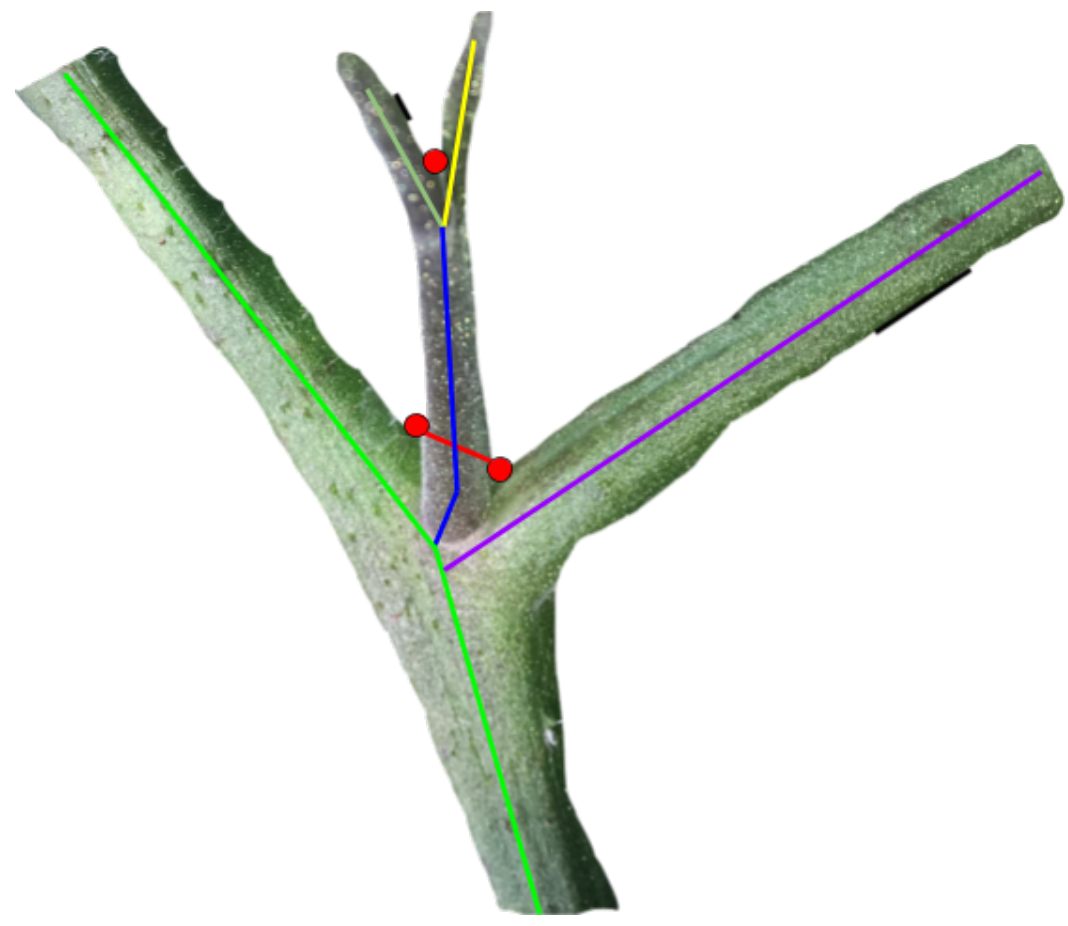

Figure 4.2: A corner pair (red) intersecting a sucker (blue)

\subsubsection{Searching Corner Pairs}

Once the valid corner pairs were obtained, each corner pair was checked to see if it correlated with a sucker. We checked each corner point of each pair to get a sense of the location of the corner point with respect to the main stem and surrounding BSs. Depending on what the corners encountered in the search, it could be determined if the corner pair was a sucker or not. If a corner encountered the main stem to the left or right, and a corner encountered a different BS below it, it was determined that the corner pair was a sucker. This simple classification of a sucker posed many problems for images that did not exhibit a simple structure like Figure 4.2. This method detected only about $60 \%$ of the suckers when the main stem was accurately identified. 
Chapter 5

\section{PROPOSED WORK}

This chapter includes the full algorithm and methods used to identify suckers in an image. Figure 5.1 provides an overview of the entire algorithm and the different components discussed in this chapter.

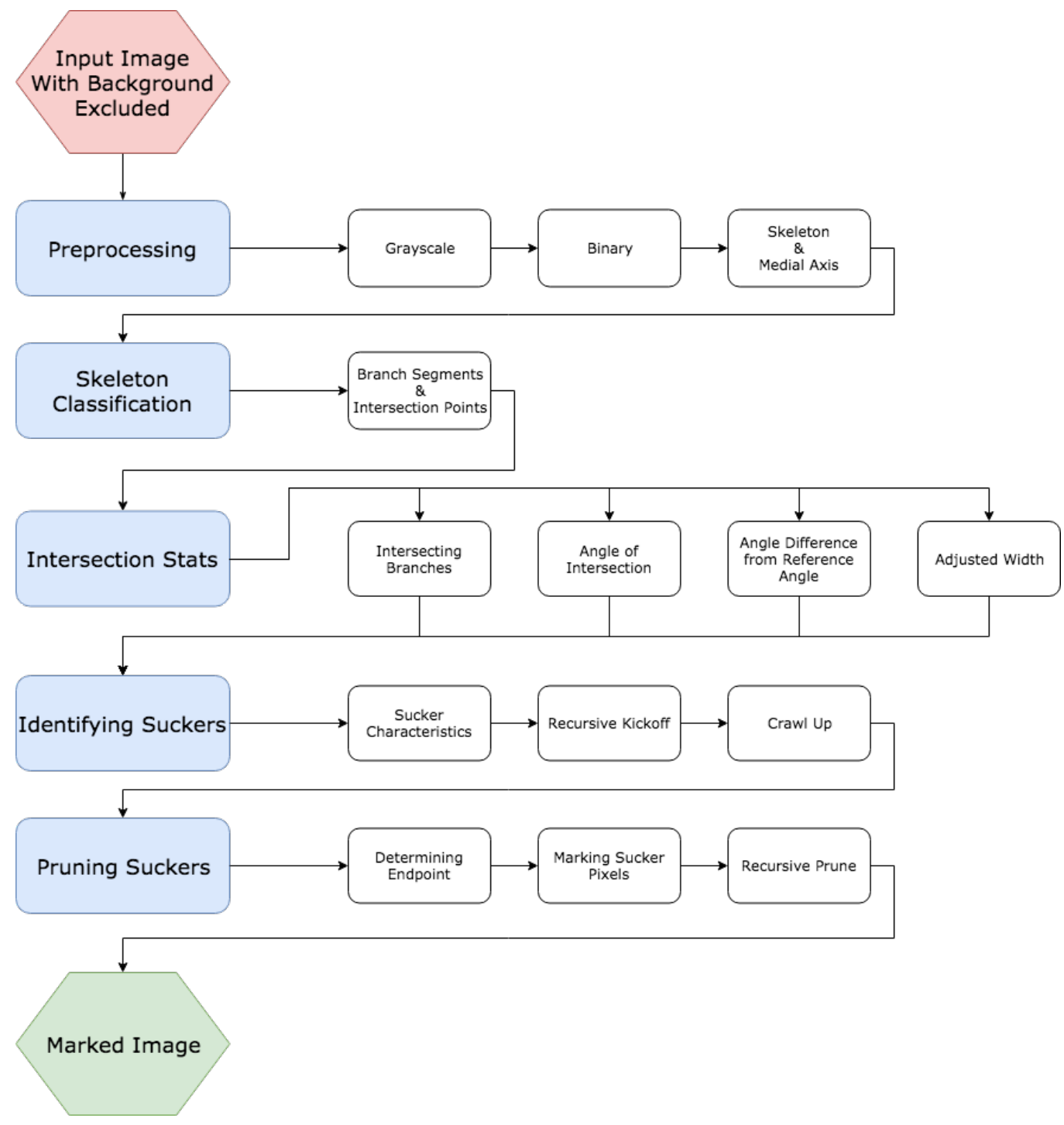

Figure 5.1: Overview of proposed algorithm 


\subsection{Preprocessing}

The input image is first grayscaled. The grayscaled image is then converted into a binary image. The binary image is finally fed into SciKit's medial axis and skeletonize functions. The skeleton and the width are returned and used in the subsequent steps.

\subsection{Skeleton Classification}

The definition of a skeletonized image is a 1-pixel wide representation of the original image. Leveraging the fact that the skeleton is only 1-pixel wide, a very simple, but accurate method was developed for classification. The next steps are to classify the skeleton into two categories: Branch Segments, and Intersection Points, which are discussed in this section.

\subsubsection{Branch Segments}

A BS is simply considered to be a group of pixels in which each pixel has two or fewer neighbors. Each BS has at least one IP at a terminating end. The different possibilities of a $3 \times 3$ grid that make up a BS pixel are shown in Figure 5.2. If the pixel only has one neighbor, it is considered part of a BS.
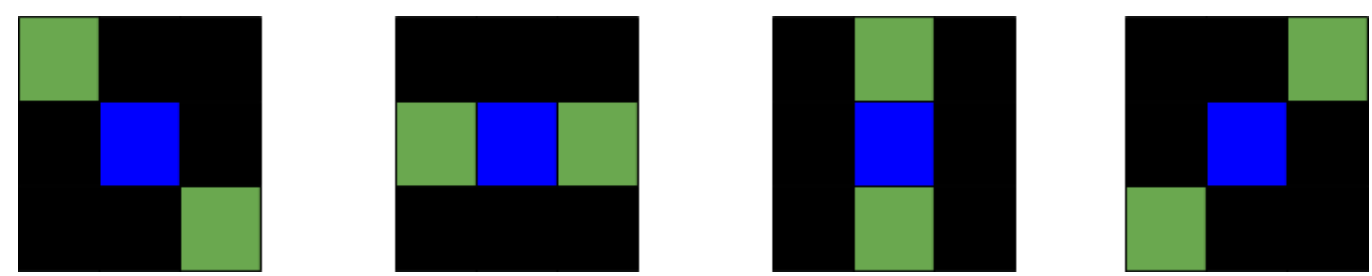

Figure 5.2: Blue pixel represents the BS pixel, the green pixels represent neighboring skeleton pixels 


\subsubsection{Intersection Points}

An IP is the inverse of a BS. It is a pixel that has three or more neighboring pixels. IPs must have at least three intersecting BSs. This is because of the definition of an IP. If an IP only had two intersecting BSs, then that would imply that the IP itself has only two neighbors, which contradicts what we define as an IP. Not all, but some of the arrangements for a IP pixel are shown in Figure 5.3
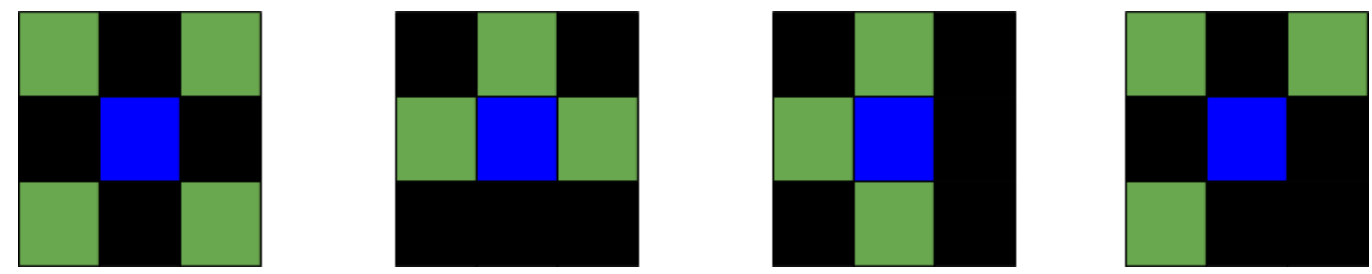

Figure 5.3: Blue pixel represents the IP pixel, the green pixels represent neighboring skeleton pixels

\subsubsection{Classification Algorithm}

A label map with the same dimensions as the skeleton image is filled in throughout the classification process to quickly look up a pixel's associated BS or IP. The label map is similar to the label map returned from OpenCV's classifying algorithms, but the label of a particular pixel is used as an index into an array containing BS and IP objects. The BS and IP objects store the coordinates of the pixels that make up each object. Recall in subsection 4.1.3, the classification algorithm was too slow for a real world application. Rather than looping through every pixel in the skeleton, we took advantage of OpenCV's and numpy's optimized algorithms to quickly classify each pixel. Numpy's where and OpenCV's connectedComponents play a major role in the speed increase. Numpy's where quickly indexes into the numpy array, and connectedComponents connects and labels all connected pixels in the image. Numpy and OpenCV are written in $\mathrm{C}$ which bypass costly python loops. Furthermore, Numpy takes advantage of locality of reference because numpy arrays are densely packed [3]. 
OpenCV utilizes Intel Integrated Performance Primitives which focuses on memory and cpu optimizations $[15,4]$. With the adjustments made, 8,500,000 pixels per second are able to be classified as opposed to the inferior 40,000 pixels per second. The algorithm for classifying the pixels is outlined in Algorithm 3. Using this method, skeleton images are perfectly classified into BSs and IPs.

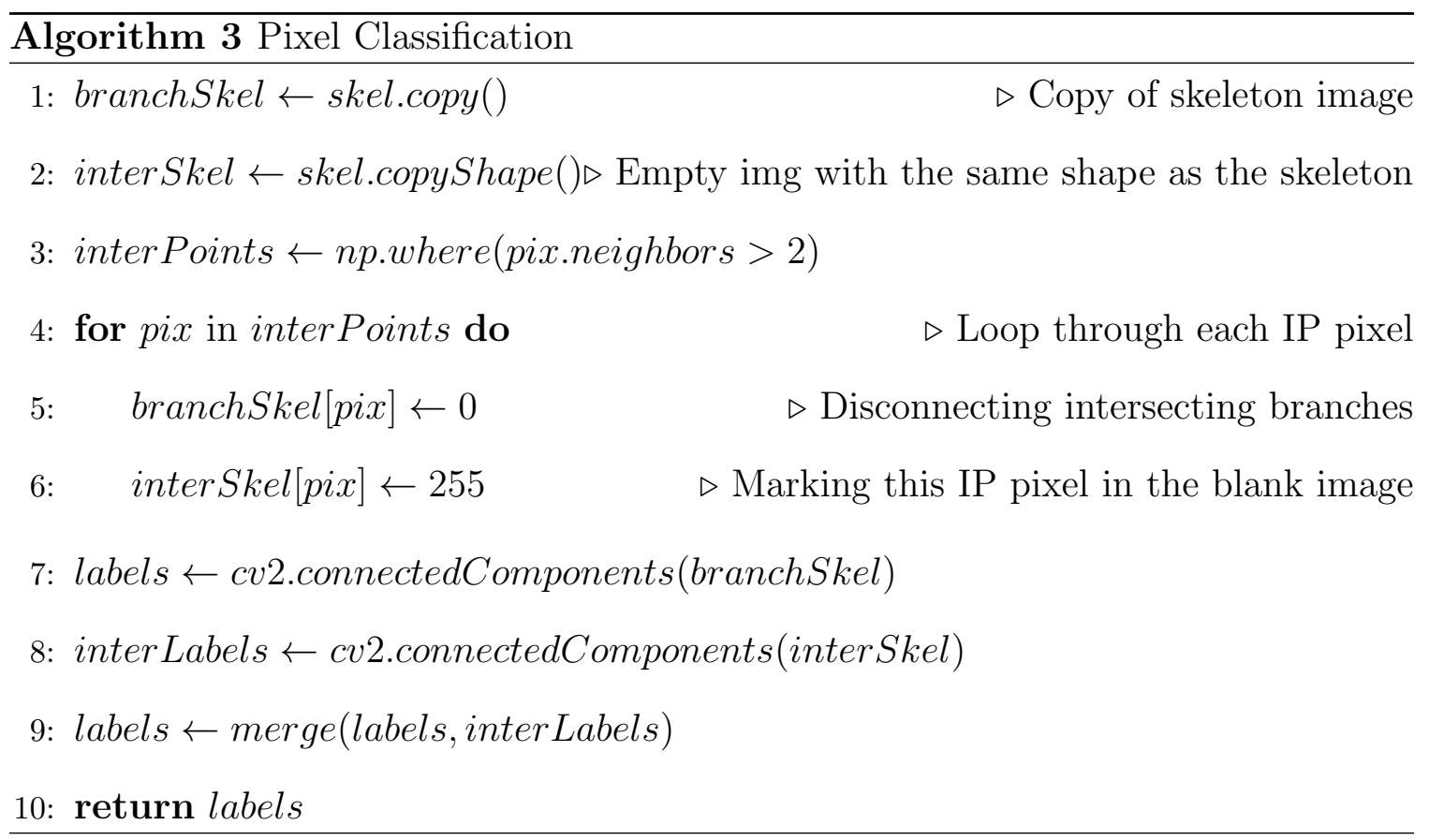

\subsection{Intersection Point Statistics}

IP statistics are an important aspect in identifying suckers, covered in section 5.5. The intersecting BSs, their angle of intersection, the adjusted BS width, and the angle difference are calculated for a particular IP. Figure 5.4 is a visual example of IP statistics. 


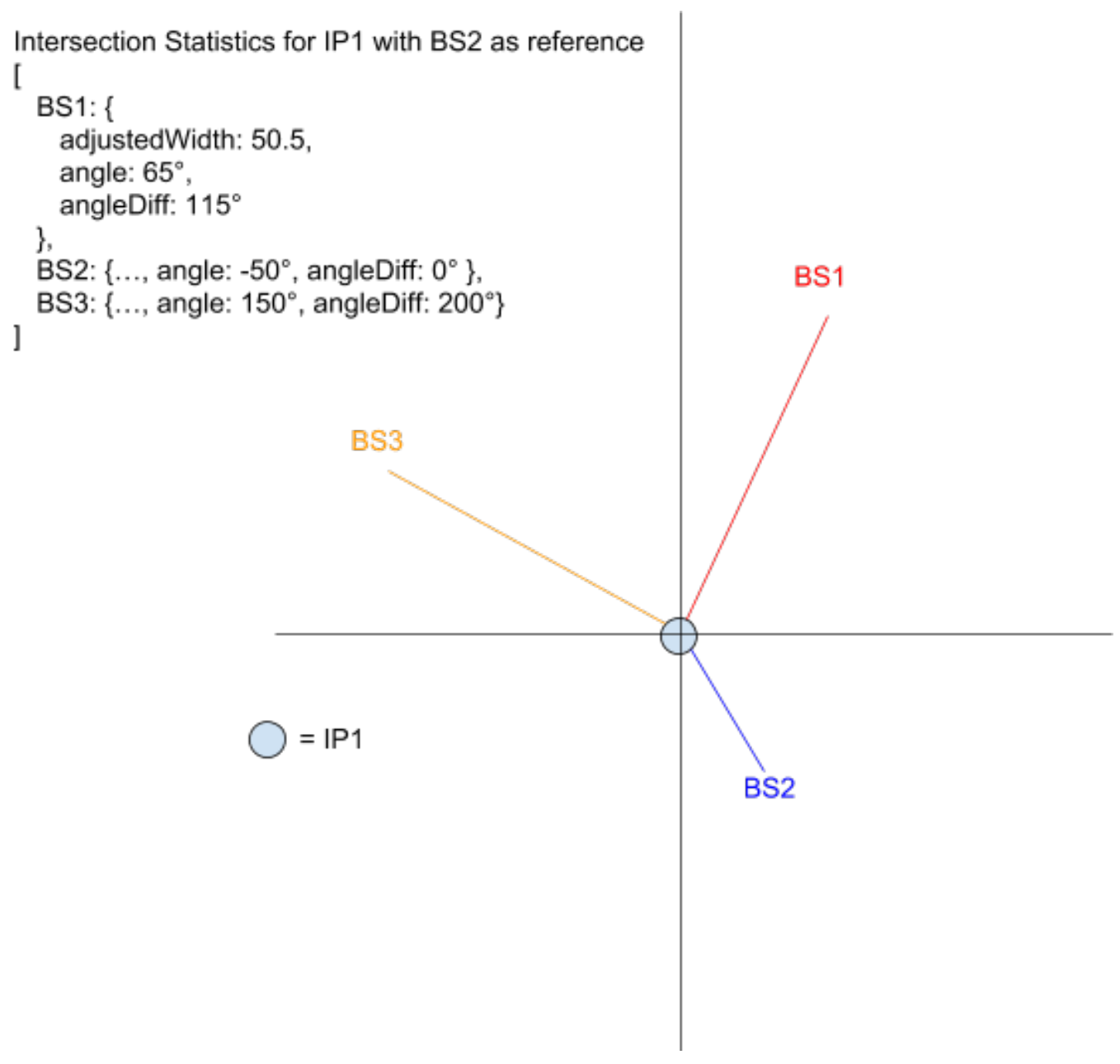

Figure 5.4: IP statistics example

\subsubsection{Intersecting Branches}

The intersecting branches are found by first calculating a distance matrix between the IP pixel coordinates and all of the pixel coordinates that make up BSs. The distance matrix contains the euclidian distance between each pair of pixels. All pairs of coordinates that result in a distance less than or equal to $\sqrt{2}$ correspond to an IP pixel directly connected to BS pixel. The IP and BS pixel coordinates are stored for later use in the calculation of the intersecting angle. After this step, all intersecting BSs are known for a particular IP. 


\subsubsection{Angle of Intersection}

The angle of intersection is an approximation of the angle at which the BS intersects the IP. To get the most accurate angle representation, we perform an outside-in search. The BS in Figure 5.5 does not exhibit a perfectly straight shape like the previous examples have shown. If the closest pixel was to be used in the angle calculation, an irregular BS would have a non-representative angle. The outside-in method starts by looking for a pixel with a euclidian distance of 40 away from the closest IP pixel to the $\mathrm{BS}$ in question. If no pixel exists at that distance, then 39 is tried, all the way down to 1 pixel or until a pixel is found at the specified distance. The algorithm of this process is shown in detail in Algorithm 4.

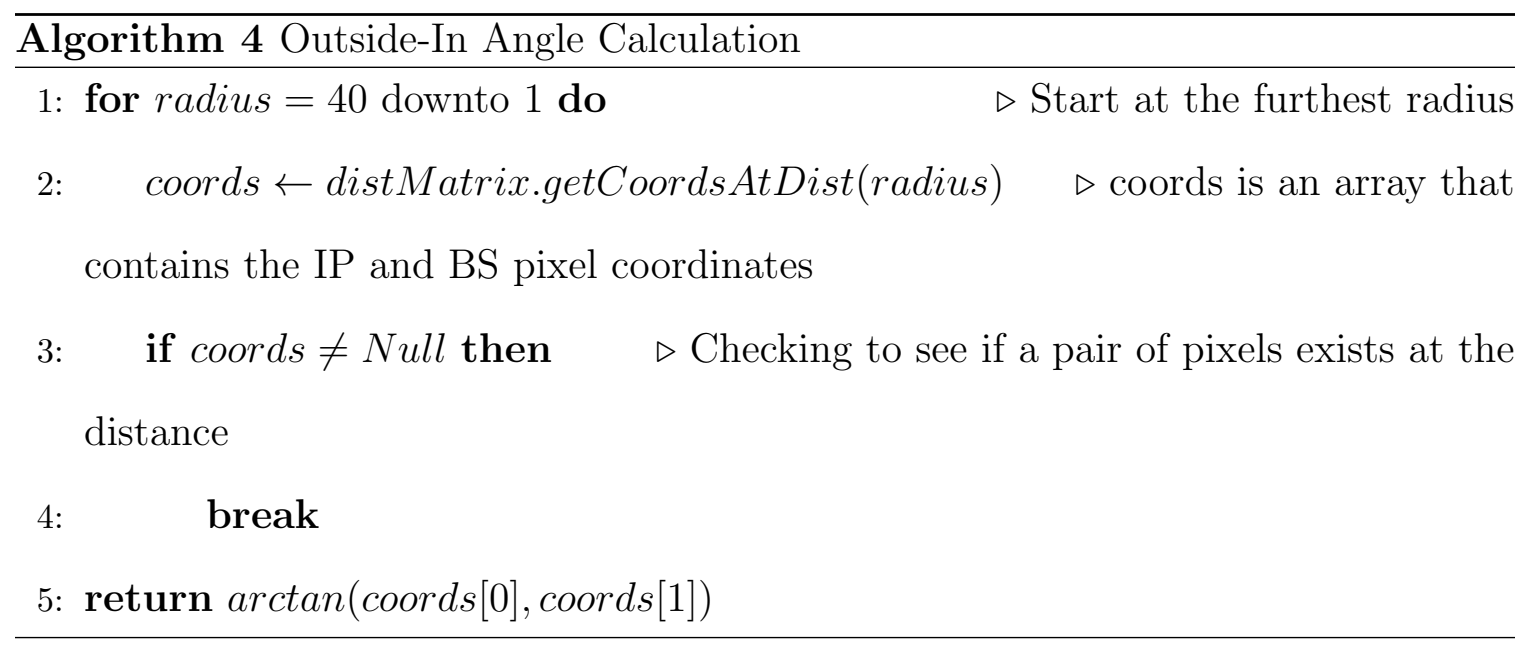




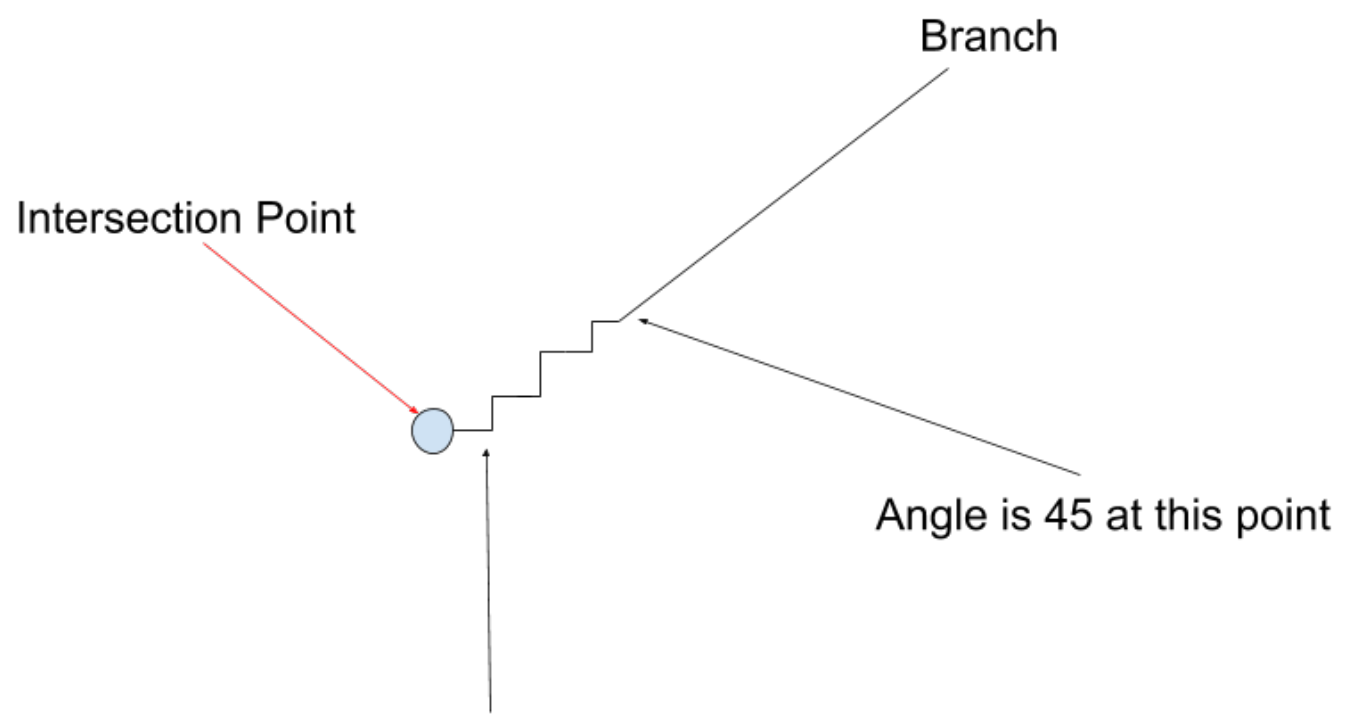

Angle is 0 at this point

Figure 5.5: Example of the outside-in approach superior to the inside-out

\subsubsection{Angle Difference from Reference Angle}

Using the angles calculated in subsection 5.3.2, angle difference between a reference $\mathrm{BS}$ and the other intersecting BSs is easily calculated. The orientation of the BS's are important when a Branch Segment of Interest (BSOI) is detected. It is important to establish "right" and "left" BSs with respect to one of the intersecting BSs. The right BS is considered as the first BS encountered when searching counter-clockwise starting at the reference $\mathrm{BS}$. The left is the second $\mathrm{BS}$ encountered. To quickly compare angle differences to determine which BS is closest counter-clockwise, Algorithm 5 shows the method used for comparing BS's angle differences. Using this method, the minimum angle difference correlates to the BS that is closest counter-clockwise, and the maximum is the furthest BS from the reference BS. Figure 5.6 shows an example of the deduction of right and left branches with respect to a BSOI. 


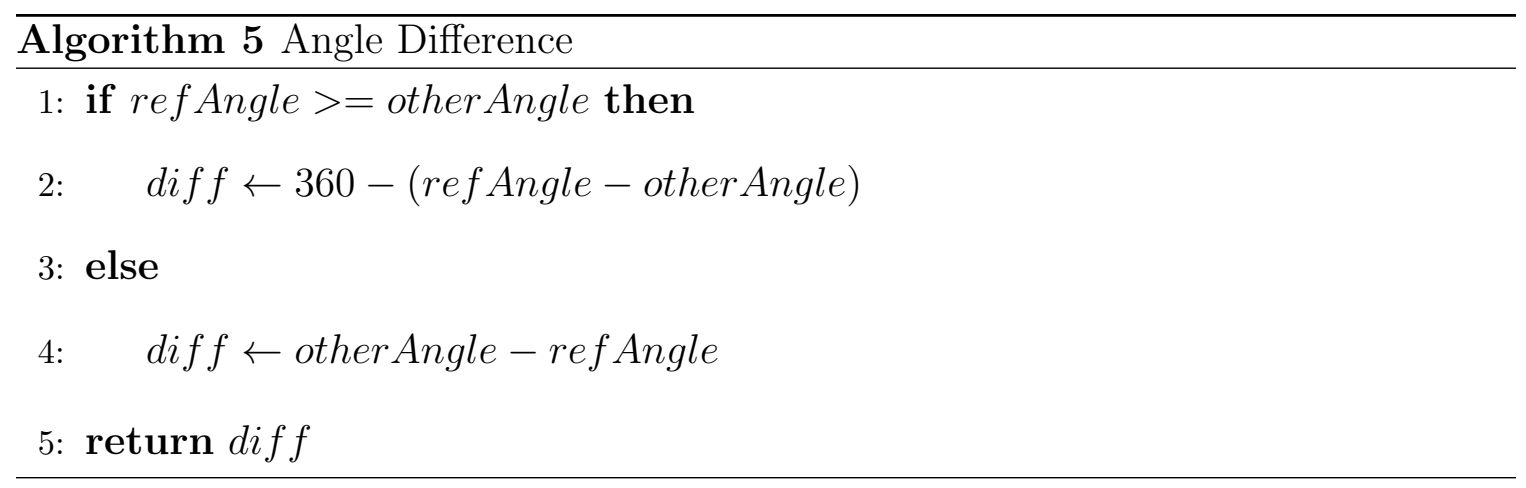

\section{Right BS}

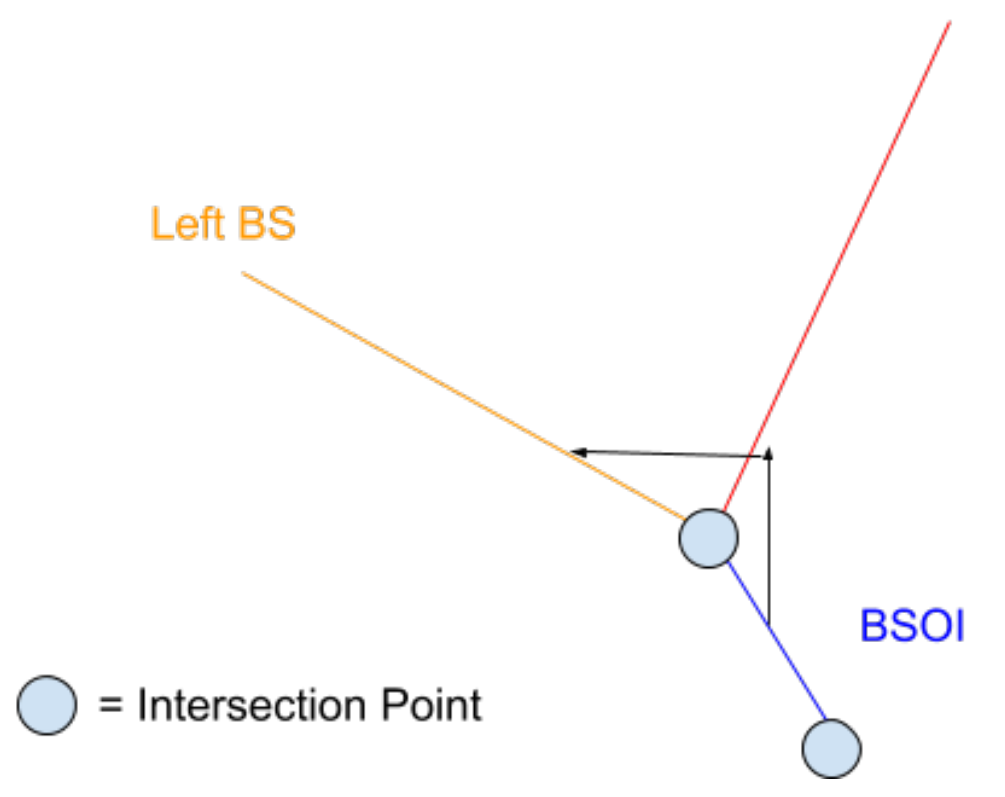

Figure 5.6: Right and left BS with respect to a BSOI

\subsubsection{Adjusted Width}

The adjusted BS width is used to get a more accurate width of a BS. The median BS width is a good basis for obtaining an approximation of the width of a uniform branch. However, if a BS makes up a large leaf, the median BS width remedies some of the skewness, but the median width is still largely skewed towards a larger median due to the wide range of width values. To counter this, we must first observe histograms of various BSs. Figure 5.8 shows BS 2 that represents the sucker with a very large leaf. 
BS 1 represents somewhat of a uniform branch, while BS 2 encompasses a leaf which has a thin, uniform-like section at the base of the main stem, but also a very large leaf towards the top of the BS. Since BS width is used in the verification of a sucker discussed in section 5.5, it is important to get an accurate width measurement. In this case, BS 2's adjusted width should be similar to the width of the uniform-like portion.

The histogram for BS 1 and BS 2 are shown in Figure 5.7. BS 1's width range is far less than that of BS 2. Furthermore, BS 2's histogram shows one bin with a majority of the data points, and the median width is far from this bin. In comparison, BS 1 exhibits more of a bell shaped distribution, while BS2 shows more of a modal distribution. BSs that represented leaves all exhibited a similar histogram to BS 2, which became the basis for calculating the adjusted width, as shown in Algorithm 6 .

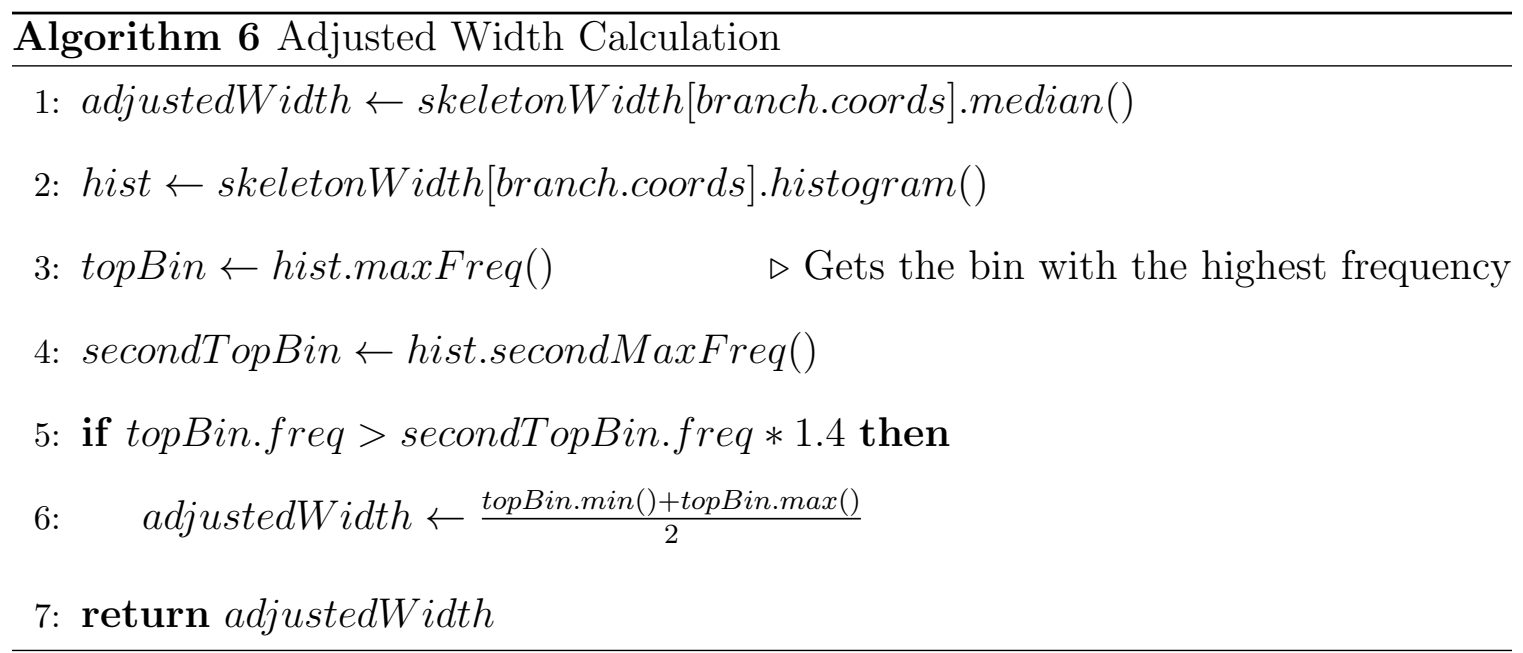




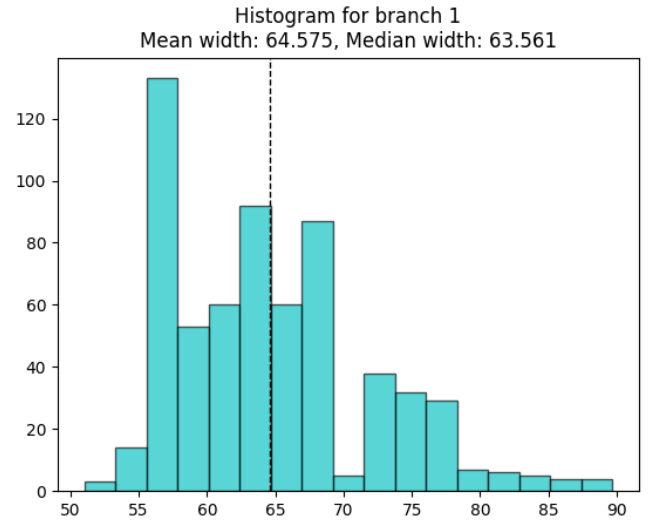

(a) Width histogram for BS1

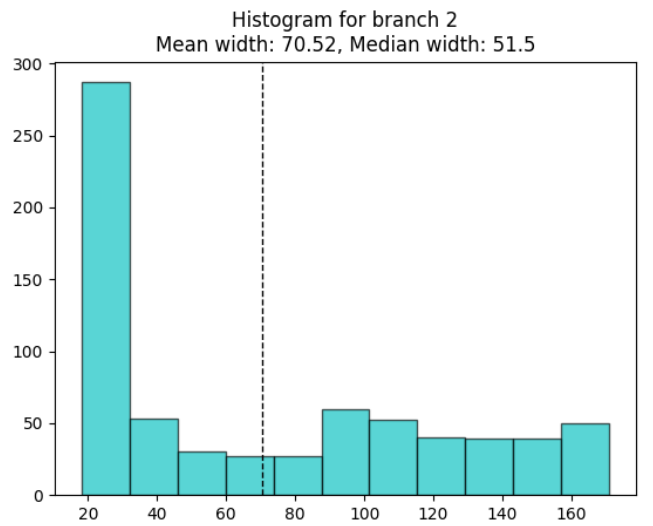

(b) Width histogram for BS2

Figure 5.7: Histogram comparisons between a uniform BS and a leaf BS

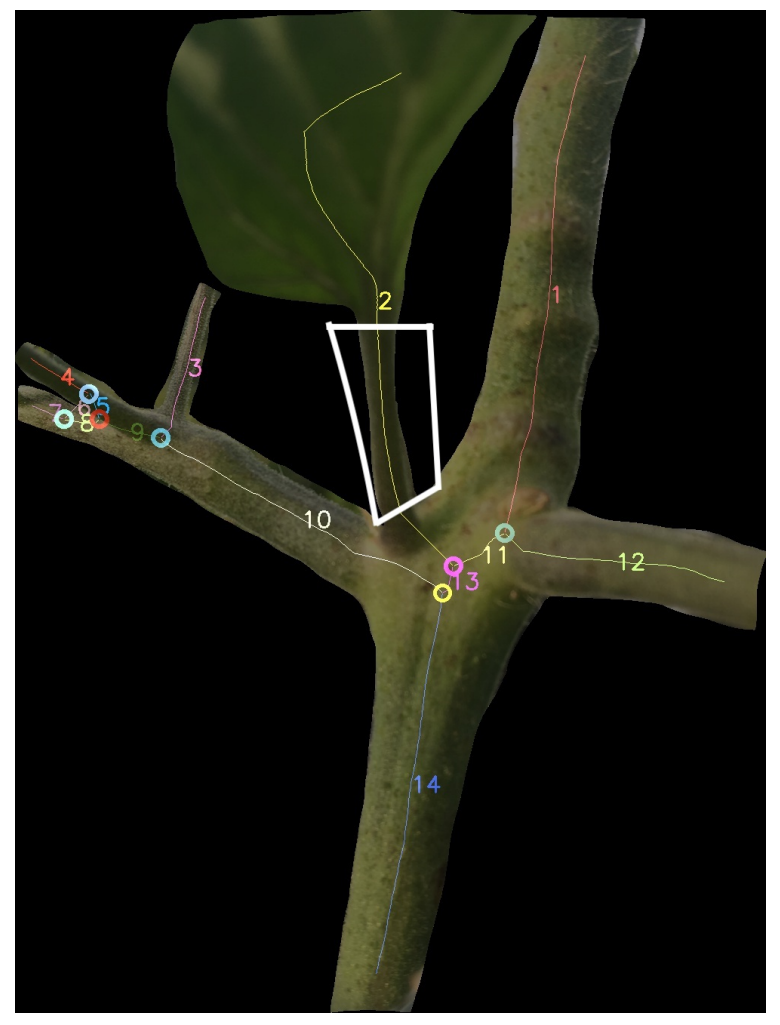

Figure 5.8: BS 2 represents a leaf, the white marks the uniform-like section of BS 2

The adjusted width is used as a final check to see if the potential sucker is indeed a sucker. The adjusted width of the potential sucker is compared against the other 
BS intersecting the same IP and the other BS not taken to get to the BSOI. This is explained in detail in subsection 5.4.3

\subsection{Sucker Characteristics}

\subsubsection{Branch Segments of Interest}

When a sucker is present in an image, very distinct physical features almost always exist in the skeleton. We refer to BSs that posses these physical features, as Branch Segments of Interest (BSOI), and they typically have a sucker at one of the terminating ends.

When compared to other BSs, BSOIs are usually short in length and have an unusually high mean width. The reason for these two characteristics are easier to see when the skeleton and original image are overlaid with one another.

Figure 5.9 provides some insight into the width and length difference in a BSOI when compared to other branches. BS 6 has the highest mean width than any other BS and it is also the shortest BS. The explanation for these BSOIs having such a high mean width is because they usually represent a portion of the main stem that splits off into another branch and or sucker. This split causes the main stem to exhibit a higher width as the stem has to continue in three directions. The shortness of the BSOI is explained by the definition of BS and IPs. If there were no sucker present in Figure 5.9, BS 6 would actually be part of BS 3, but since a sucker is present, a short BS must be present between BS 3 and BS 7 as there are two IPs. This led to two metrics being used as the tests for determining if the BS was indeed a BSOI. 


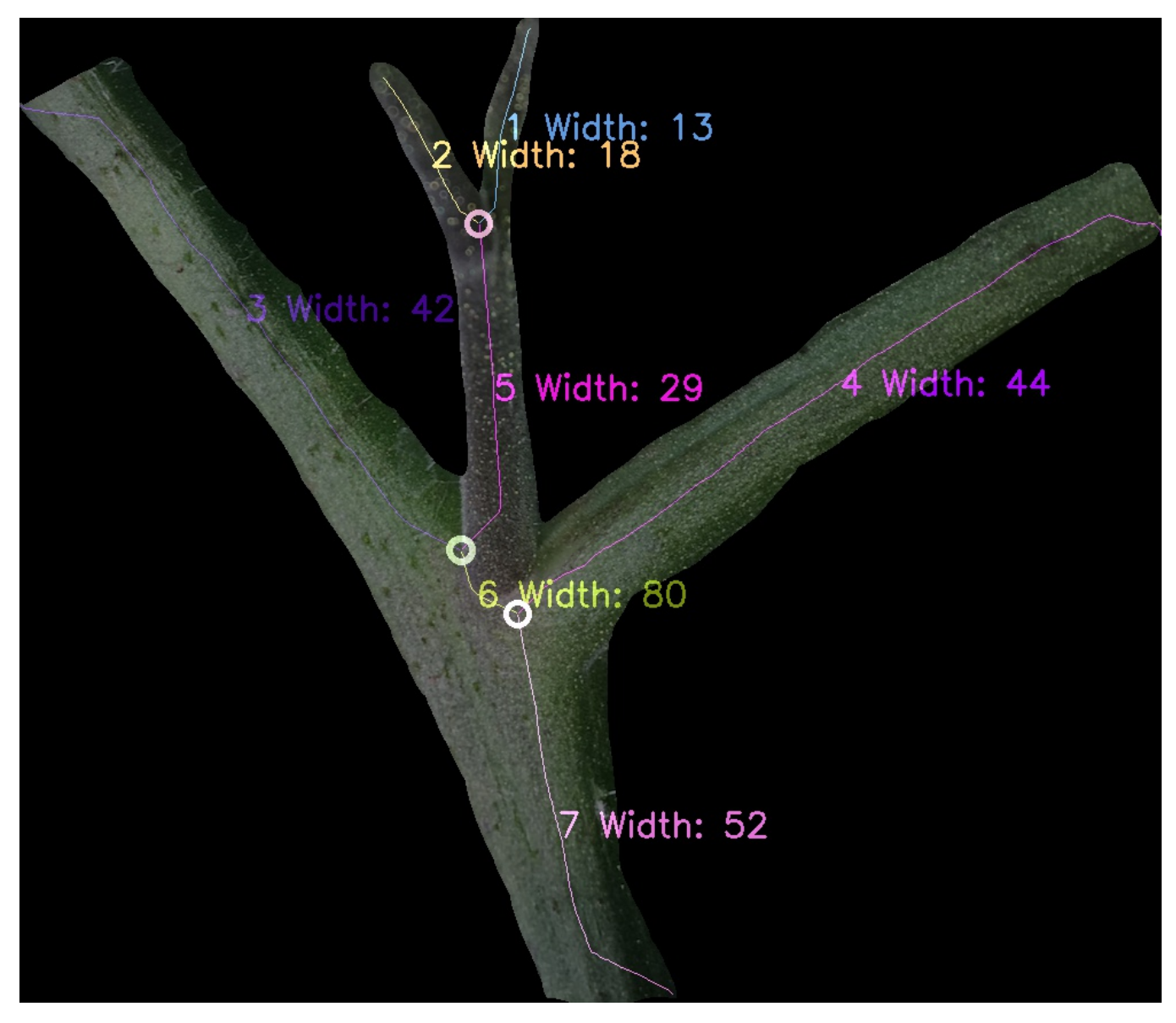

Figure 5.9: Overlaid image of the skeleton and the original

The mean width of a BS is easily calculated since the skeleton width is returned along with the skeleton. When the test images were experimented with, most BSOIs exhibited a mean width greater than $76 \%$ of the maximum BS mean width. The metric agreed upon was the meanWidthRatio and a simple equation for calculating this for each branch is shown in Equation 5.1

$$
\text { meanWidthRatio }=\frac{\text { skeletonWidth[branch.coords].mean }()}{\text { allBranchWidths.max }()}
$$

The other metric involves the length of the BS. Since a BS can take multiple turns and is rarely straight, the distance between the start and endpoints of a BS cannot be taken as the length of $\mathrm{BS}$. Instead, the number of pixels that make up the $\mathrm{BS}$ is 
used in the calculation of the metric, BSPixelRatio as shown in Equation 5.2. The images showed that almost all BSOIs had a BSPixelRatio less than 7\%.

$$
\text { BSPixelRatio }=\frac{\text { branch.coords.length }}{\text { totalSkeletonPixels }}
$$

Thus, a BS is considered a BSOI if it passes the conditions in Equation 5.3.

$$
\text { BSPixelRatio }<=0.07 \& \text { meanWidthRatio }>=0.76
$$

\subsubsection{Search Branch Segments}

Search Branch Segments (SBS) are all BSs that are not considered BSOIs. These branches are recursively searched for BSOIs, which is discussed in section 5.5

\subsubsection{Potential Sucker and Verification}

A potential sucker is defined as being the right BS if the BSOI is the left BS from the source and vice versa if the BSOI is the right BS from the source. The non-potential sucker is the BS that is not the potential sucker. The potential sucker's adjusted width must be less than the non-potential sucker's width or the other BS's adjusted width to be considered a sucker. If the potential sucker's width is greater than both of those BSs, then it is not considered a sucker. The right, left, potential and nonpotential sucker are better visualized in Figure 5.10. The BSOI is the right BS from the source, so the potential sucker is the left BS from the BSOI. 


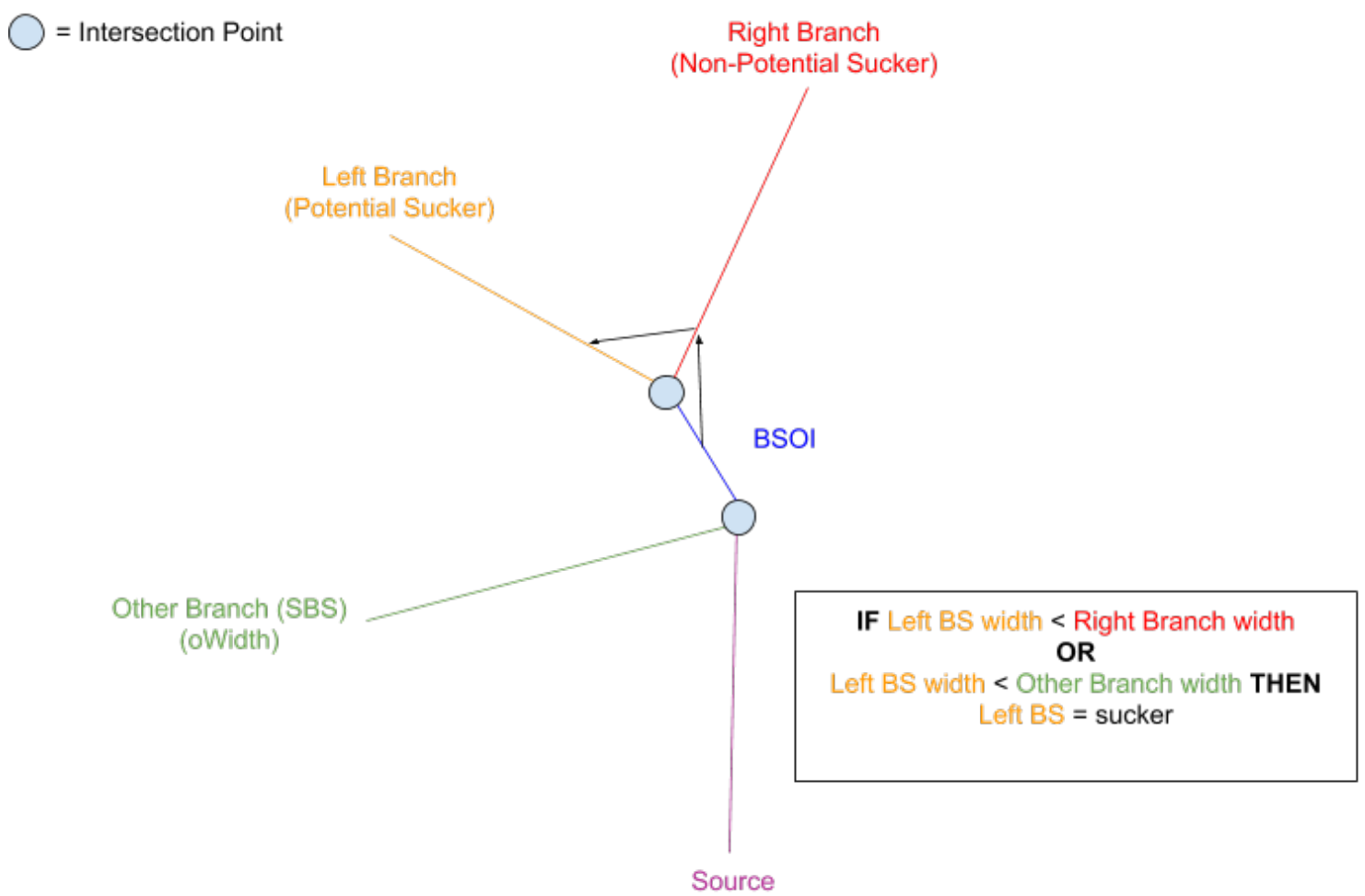

Figure 5.10: Example of potential sucker selection and verification

In the test images we found one instance that prompted the addition of another condition that defined a potential sucker. If an IP has more than three intersecting BSs, the middle-most BS is determined as the potential sucker and the BSs directly to the left and right of the potential sucker are used in the verification process described above. Figure 5.11 is an example of this scenario. 


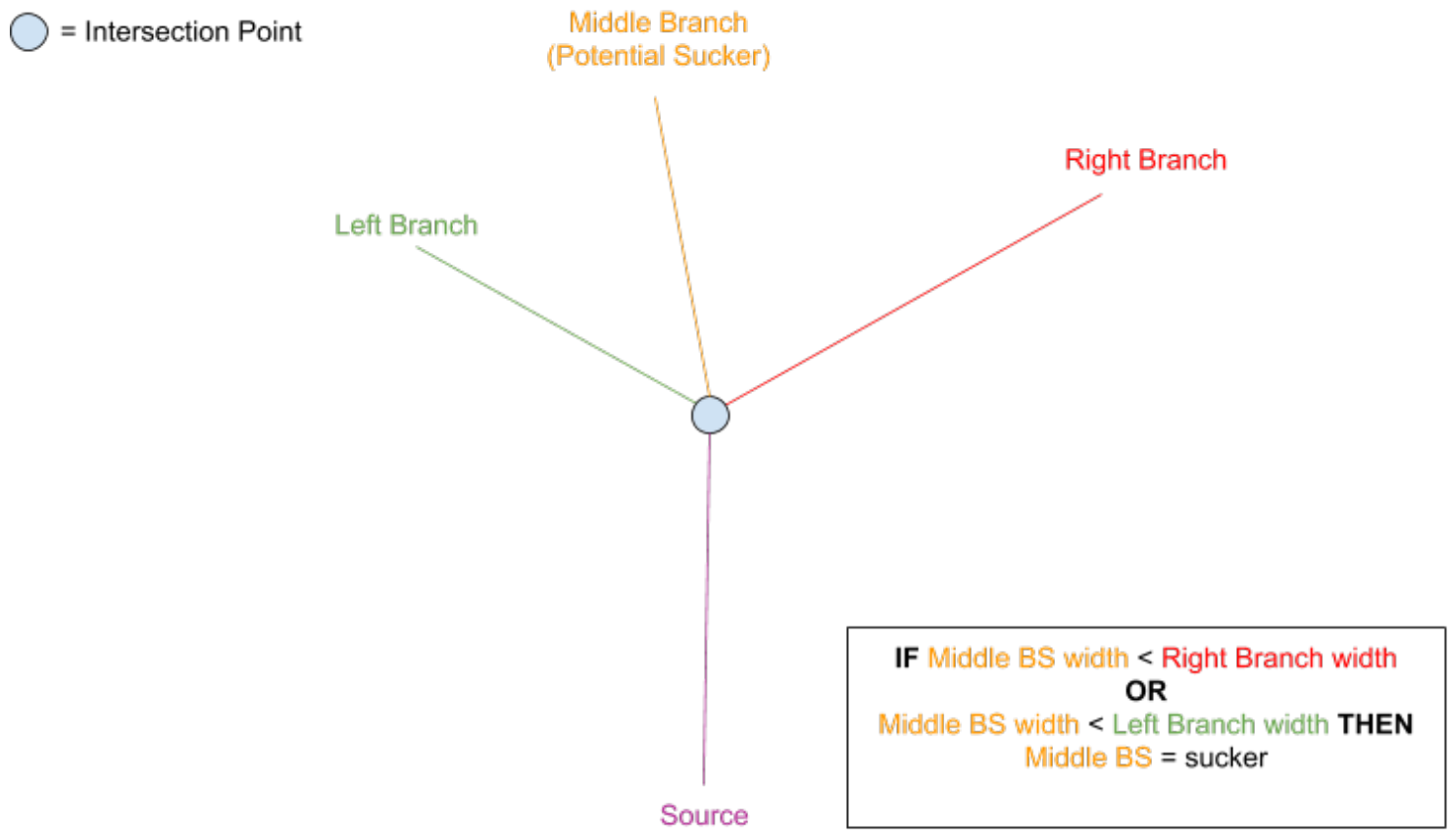

Figure 5.11: Four intersecting BSs with middle BS as the potential sucker

\subsection{Identifying Suckers}

This section describes the algorithm that searches the entire classified skeleton from section 5.2 and uses the metrics discussed in section 5.3 and 5.4 to identify and locate suckers.

\subsubsection{Recursive Kick Off}

The recursion is started by first finding the BS with the highest y value coordinate. This correlates to the BS that is lowest in the image. Once, the lowest BS is found, crawlUp is called on BS, starting the recursive method. 


\subsubsection{Crawl Up}

Once crawlUp is called on the starting BS, the entire skeleton will be searched for suckers. The entire crawlUp method is laid out in Algorithm 7, but the general process is:

1. For the current branch, get the IP that is not in the global list of parsed IPs.

2. Add the IP to the global list of parsed IPs.

3. Check each BS intersecting and if it is a BSOI, call crawlUp on it. If not, add it to a list to be searched.

4. If the current branch is a BSOI, check the appropriate BS to see if it passes the sucker test.

- If the BS passes the sucker test, call prune on the BS.

- Otherwise, add the BS to the list to be searched.

5. Call crawlUp on all BSs in the list to be searched. 


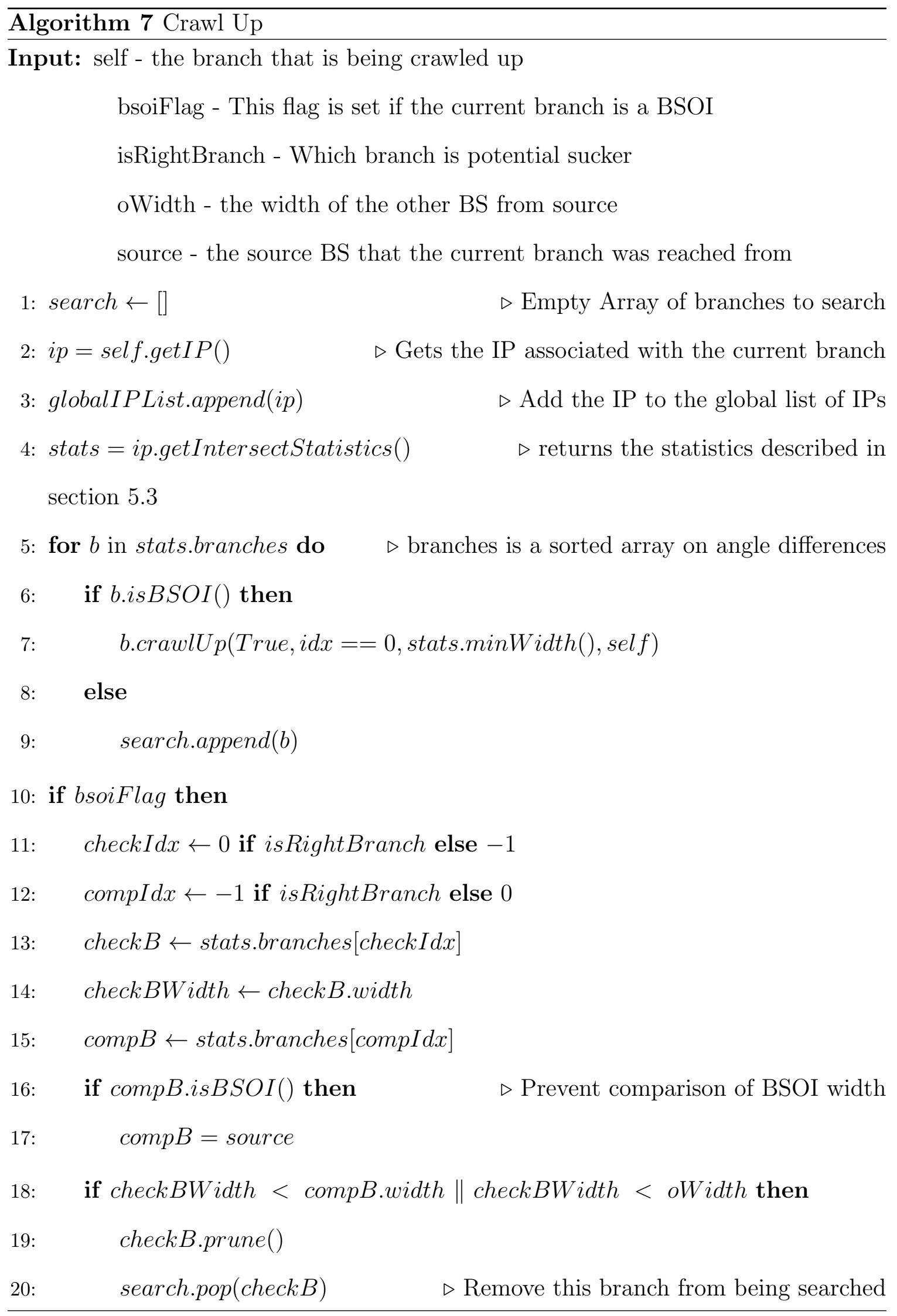




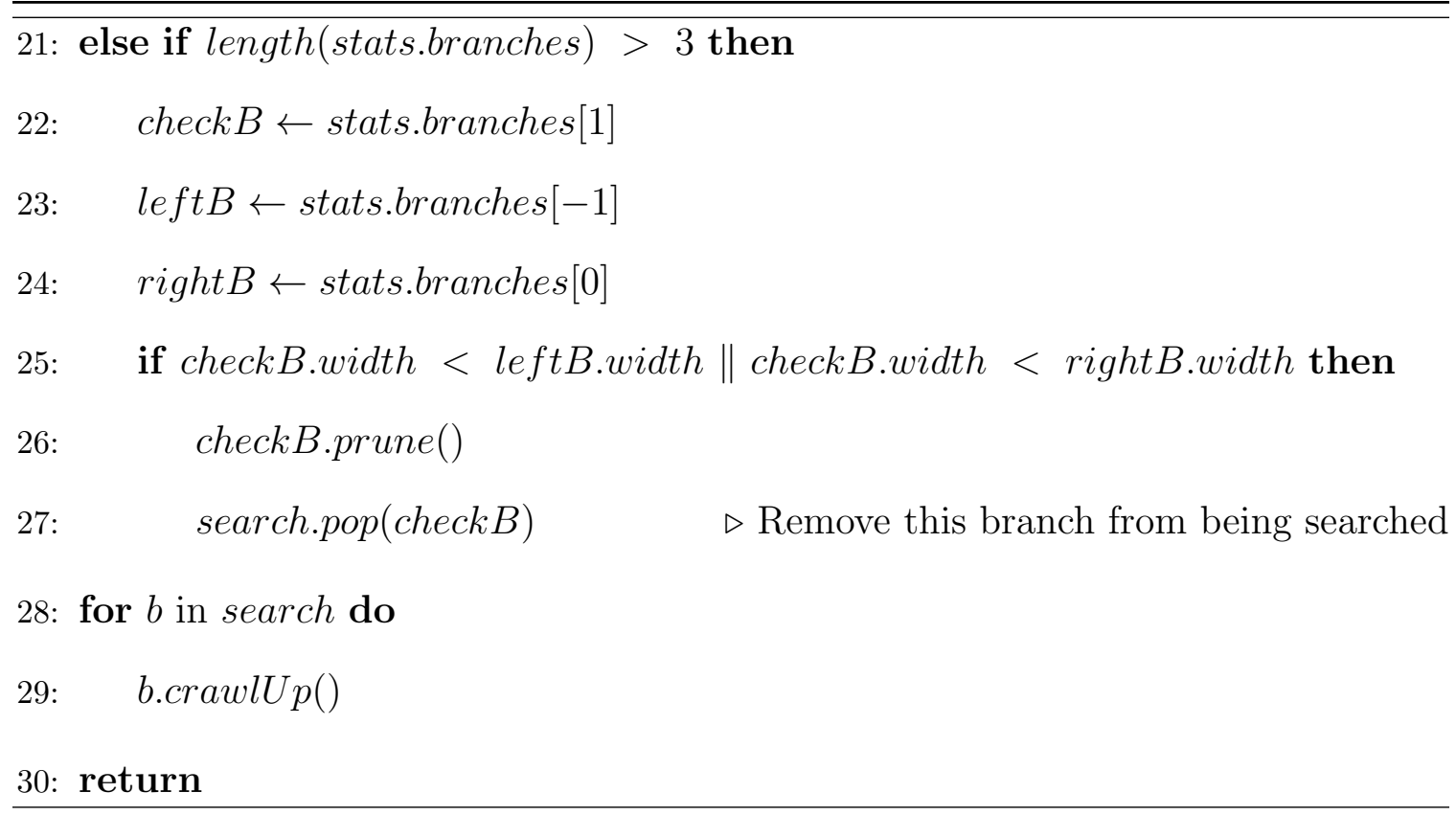

\subsection{Pruning Suckers}

\subsubsection{Identifying Sucker Pixels}

Once a $\mathrm{BS}$ is identified as a sucker, the pixels in the original image that belong to the sucker need to be marked. Recall that, for every pixel in the skeleton, there is an associated width value in the skeleton width array. However, this is only a scalar value and does not give any information about which direction from the skeleton pixel the distance should be added to. To solve this, every pixel's approximate slope is calculated using the same outside-in approach as Algorithm 4, except the starting radius is 3 instead of 40 . In this case, an approximate angle is desired for the pixel, and not the entire BS. After the angle is obtained, the $\mathrm{x}$ and $\mathrm{y}$ component can be calculated in Equations 5.4 and 5.5.

$$
\begin{aligned}
& x \text { Component }=\text { absolute }(\text { cosine }(\text { angle })) * \text { skeleton } W i d t h[\text { coordinates }] \\
& y \text { Component }=\text { absolute }(\operatorname{sine}(\text { angle })) * \text { skeletonWidth }[\text { coordinates }]
\end{aligned}
$$




\subsubsection{Determining Endpoint}

The skeleton width and BS's adjusted width are used in conjunction to determine which pixels to mark red. Although a BS is identified as a sucker, not all pixels within that BS should be marked for pruning, since the BS overlaps with the main stem and supporting branch. Ideally, the base of the sucker would be where the sucker is marked. The BS is marked red from the bottom down, and once a skeleton pixel is found that is within $10 \%$ of the adjusted width of the $\mathrm{BS}$, the marking of pixels stops. The effect of an ending point is shown in Figure 5.12.

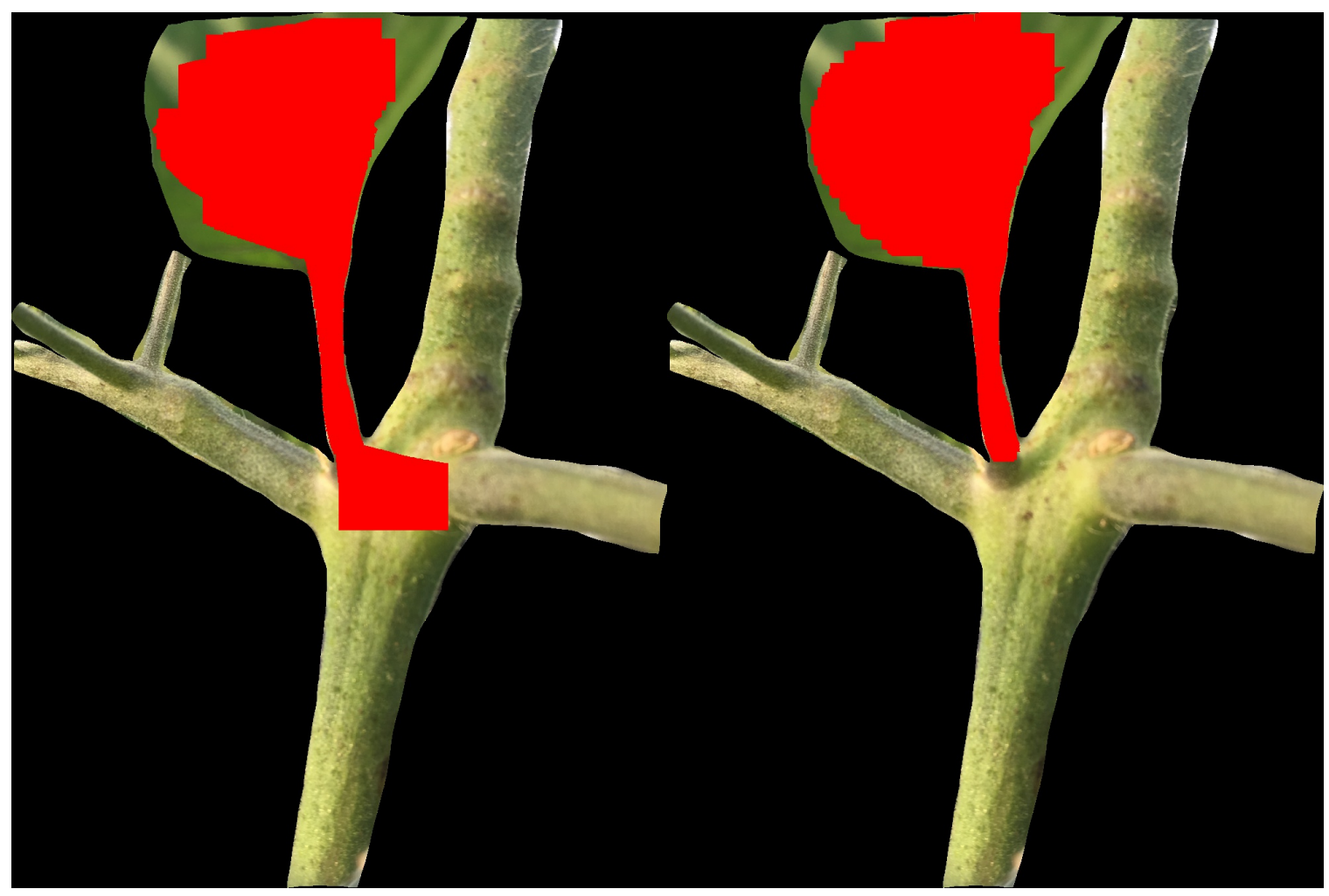

(a) Without pruning endpoint

(b) With pruning endpoint

Figure 5.12: Comparison of the sucker marked with and without endpoint 


\subsubsection{Recursive Prune}

It is possible for a BS to be identified as a sucker, and have more BSs connected to the sucker BS. For example, Figure 5.13 shows the sucker, BS 4, splitting off into BS 1 and 3. To remediate this, a recursive function, similar to Algorithm 7 is used to prune all associating BSs.

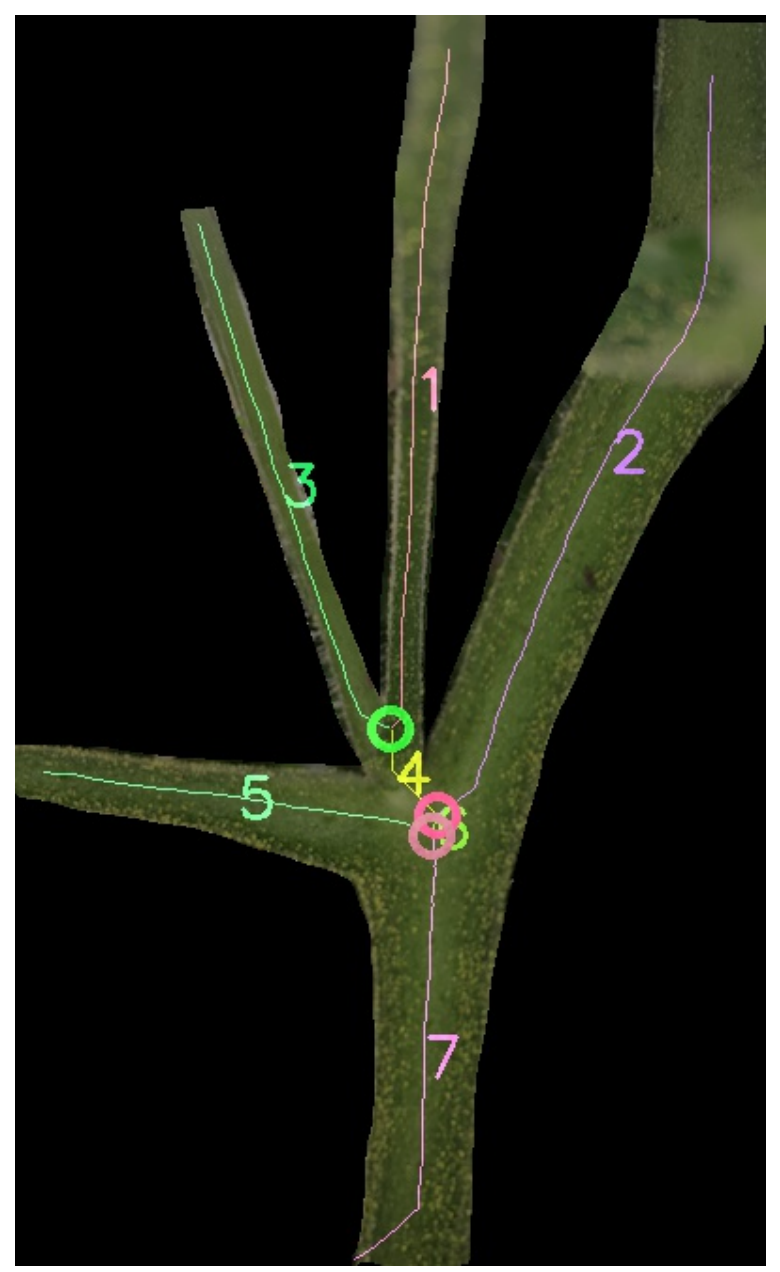

Figure 5.13: Example of a complex sucker where recursive pruning is needed 


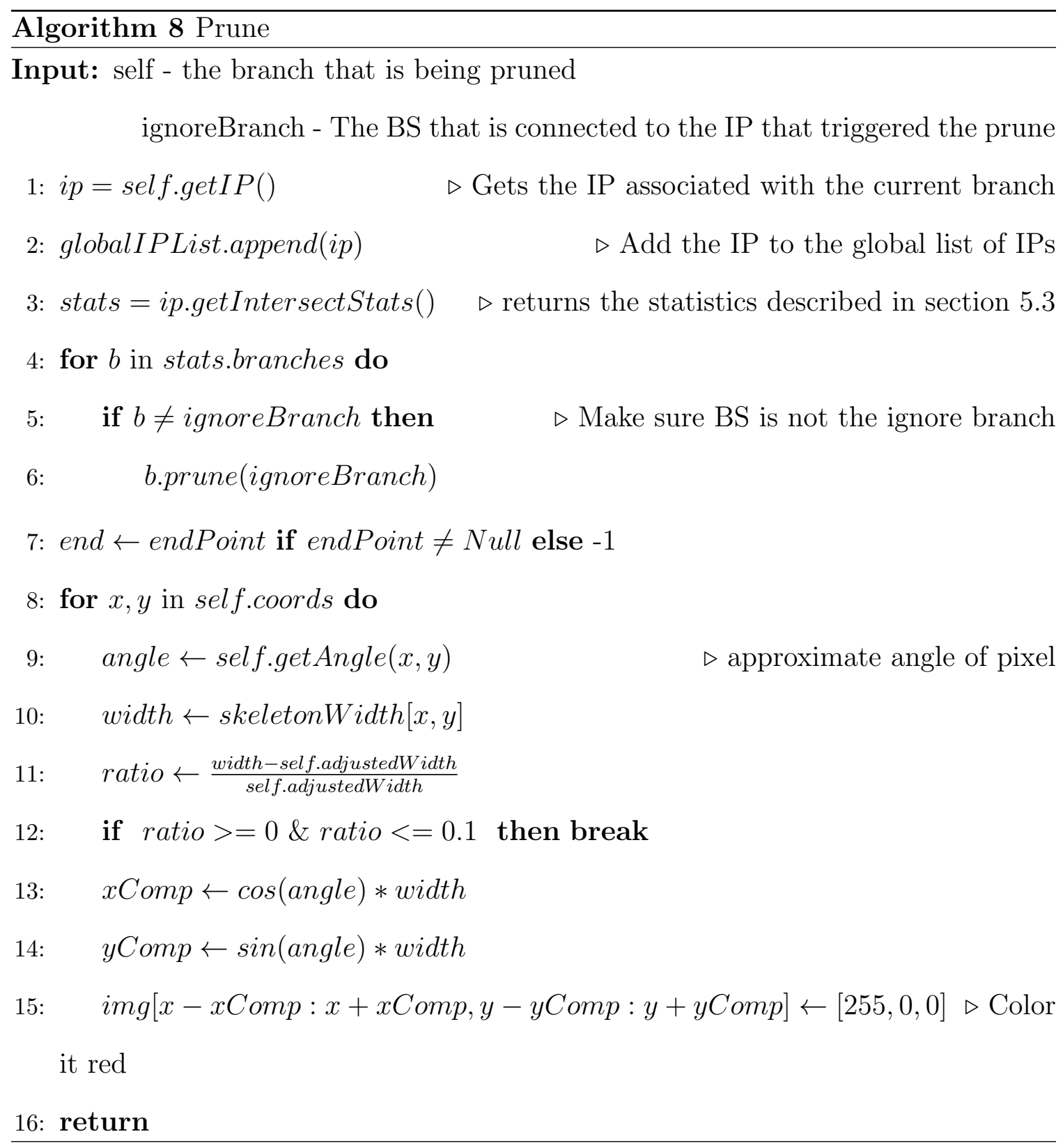


Wide sucker 
Chapter 6

\section{EVALUATION}

The main objective of this thesis is to accurately identify tomato suckers given an input image with the background excluded. This chapter covers the results using 100 test images and their downsampled counterparts by using the algorithm implemented in Chapter 5. The test images were downsampled from $10 \%$ to $90 \%$ of their original size to test the scale invariance of the identification algorithm. The background was excluded by cropping the areas of interest. Analysis of the testing results reveals the strengths and limitations of this work's proposed algorithm.

\subsection{Identifying Sucker Branches}

The algorithm's ability to identify the correct BSs as suckers is discussed and analyzed in this section. Out of the 100 images, there were a total of 122 suckers. The test dataset includes images with one or multiple suckers, straight branch suckers, complex leaf suckers, and thin and thick suckers. Some of the correctly labeled images, as well as instances where the algorithm failed, are both discussed in this section.

\subsubsection{Original Test Dataset}

The original images were taken with a $12 \mathrm{MP}$ camera, resulting in all the images being 3000 x 4000 pixels. All of the images were collected in the same day between 2 and 4 PM from Dr. Liu's greenhouse in February 2018. The greenhouse is $40 \mathrm{x} 12 \mathrm{ft}$ and contains about 300 individual tomato plants that makeup 50 different varieties of indeterminate tomatoes. Since the tomato varieties were not physically marked in the greenhouse, the actual tomato varieties that the test images encompass are 
unknown.

A high success rate was achieved with the algorithm developed in this thesis. Virtually all suckers were identified and no instances of false positives occurred. Table 6.1 shows the true positives detected in the 100 test images. The algorithm failed to detect suckers in only three images. The reasoning behind each failure is discussed later in this section.

Table 6.1: Accuracy for the 100 original images

\begin{tabular}{|c|c|c|}
\hline & Images & Suckers \\
\hline Correctly Labeled by Algorithm & 97 & 118 \\
\hline Total & 100 & 122 \\
\hline True Positive & $97 \%$ & $96.7 \%$ \\
\hline False Positive & $0 \%$ & $0 \%$ \\
\hline
\end{tabular}

One downfall to the approach developed in this thesis is the lack of depth perception and orientation. The algorithm inputs a normal image and assumes the image in 2-dimensional space. As seen in Figure 6.1, there are two suckers, BS 6 and 8, branching off of BS 5 and 9. BS 5 actually encompasses two branches. The main stem is directly behind BS 5. The process of taking the BSOI and looking for a sucker, as covered in subsection 5.4.3, does not pass the test in this photo. BS 7 is considered the BSOI, and according to the criteria in subsection 5.4.3, BS 5 is considered the potential sucker, but it passes the width tests so it is not marked as a sucker. 


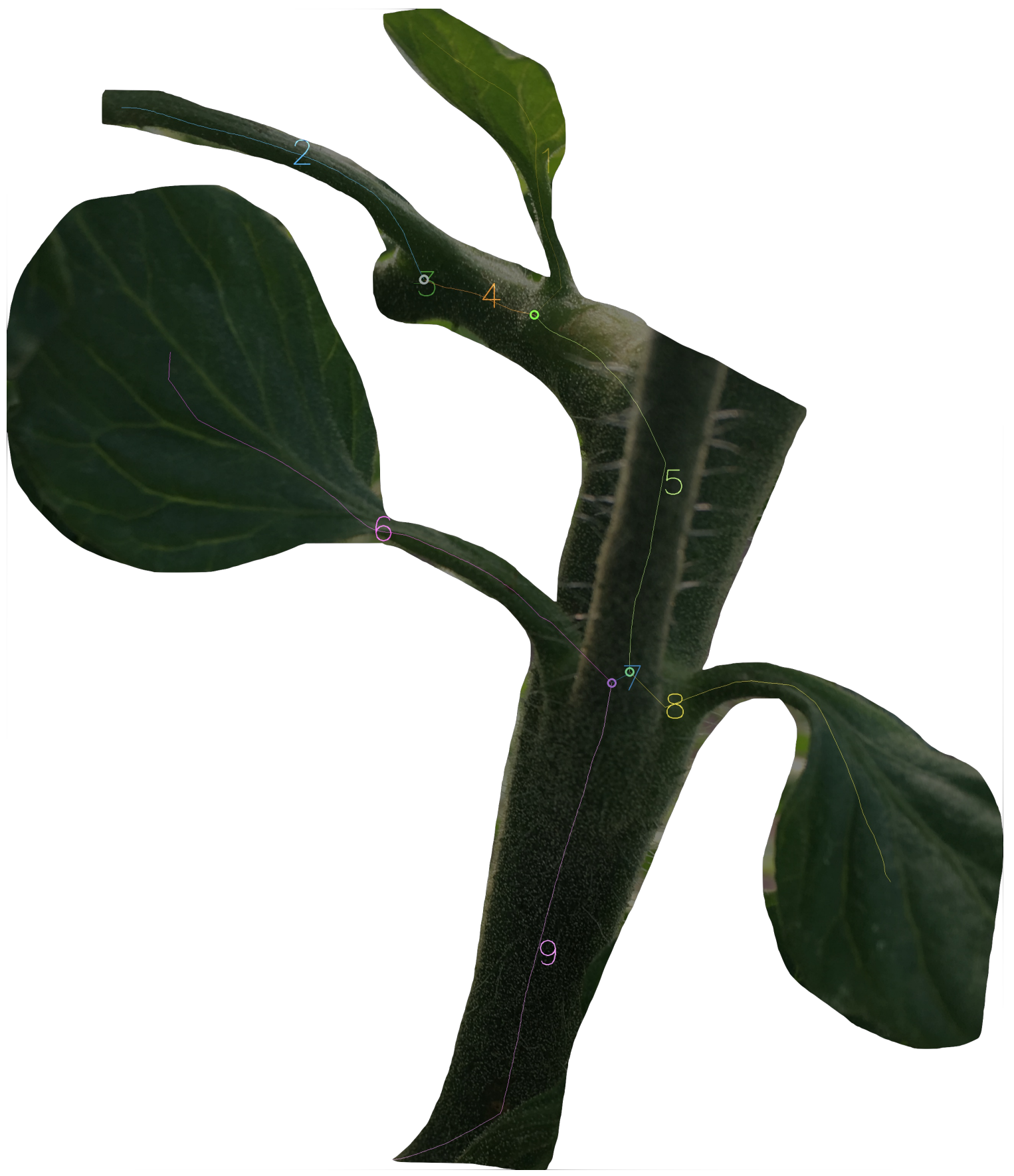

Figure 6.1: Both suckers missed due to orientation of image

Figure 6.2 shows one of the suckers being missed. This is due to a complex skeleton and a lack of the sucker characteristic described in subsection 5.4.3. The problem arises from the layout of the skeleton. BSOI 9 follows the algorithm and BS 
3 is correctly detected as a sucker. However, BSOI 8 follows the algorithm and BS 2 is the potential sucker. BS 2 passes the width tests so it is not marked as such.

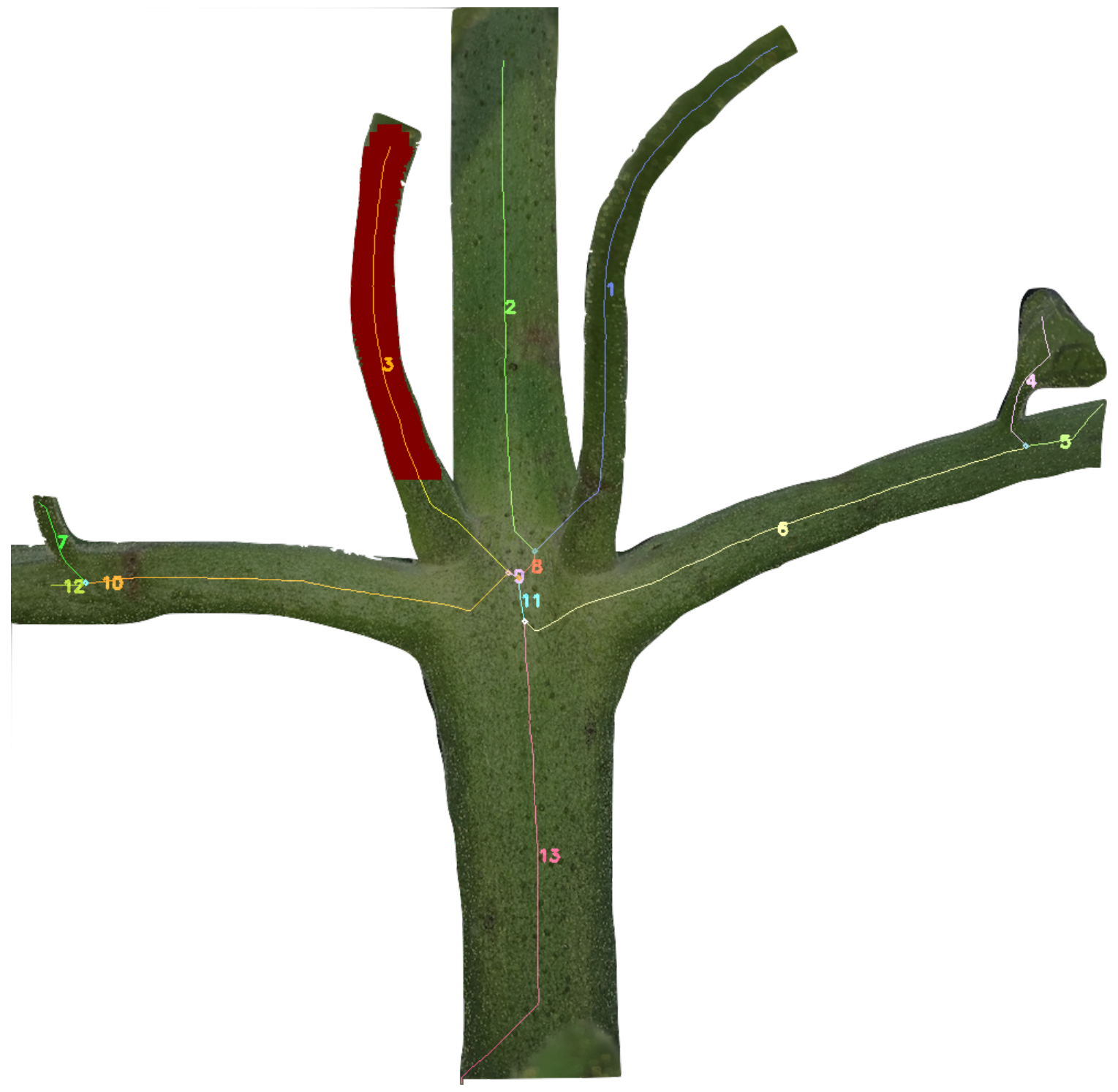

Figure 6.2: One of the suckers is missed due to failure of sucker characteristic

Figure 6.3 is the third image that was incorrectly labeled. The meanWidthRatio threshold of $76 \%$ was never satisfied because BS6 had a width ratio of $71 \%$. It never met the threshold because BS 7 is a BSOI with a very large mean width. This prevented BS6 from being detected as a BSOI. BS 2 passed the width tests so it was 
never marked as a sucker. This image is a good example of our sucker verification method working, but our definition of a BSOI failing. Lowering the BSOI threshold was not an option to remedy the problem because it would cause far too many false positive BSOIs.

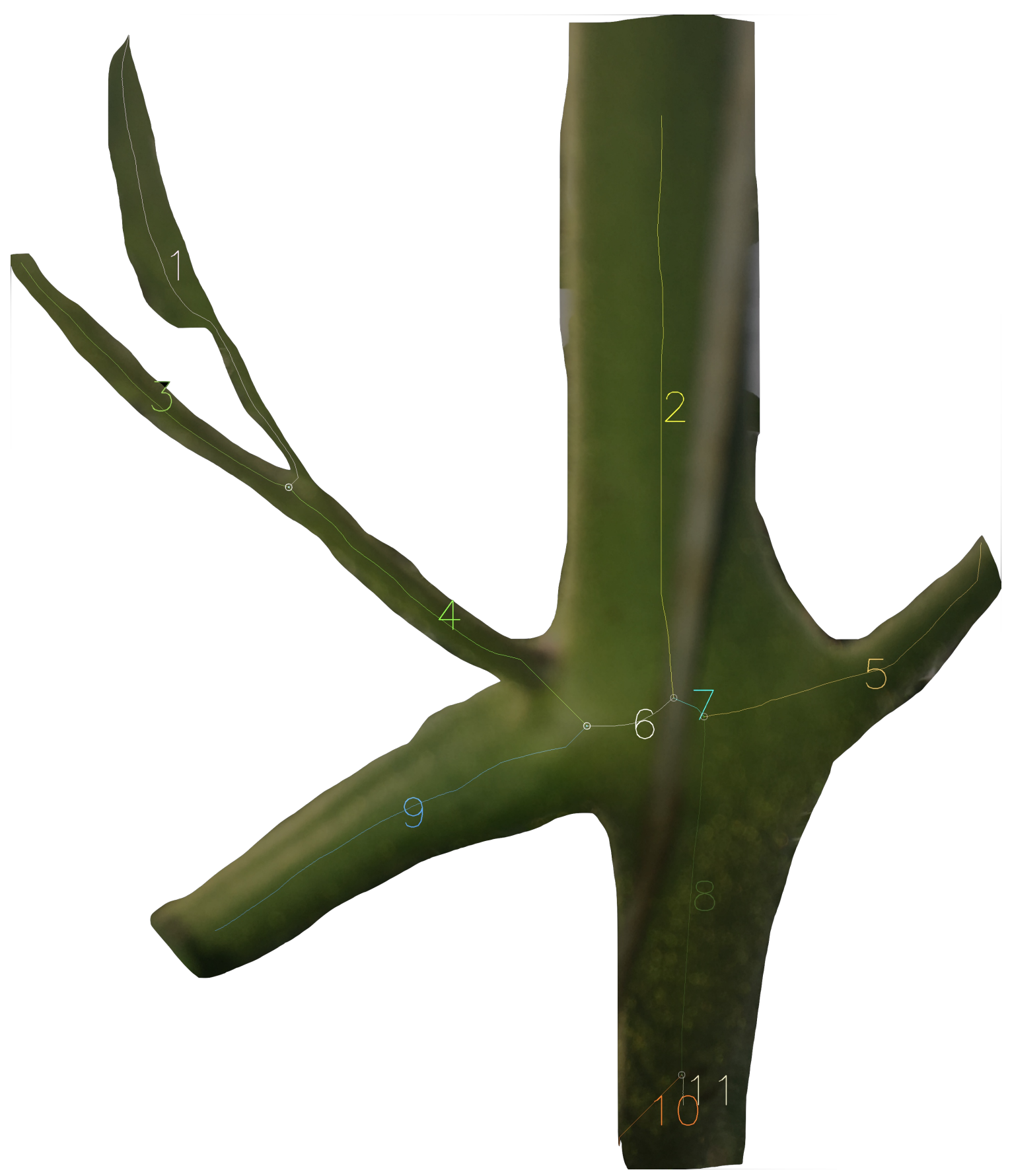

Figure 6.3: Missed BSOI resulted in a missed sucker 
Figures 6.4, 6.5, 6.6, 6.7 are examples of images that had multiple suckers spawning from the same position.

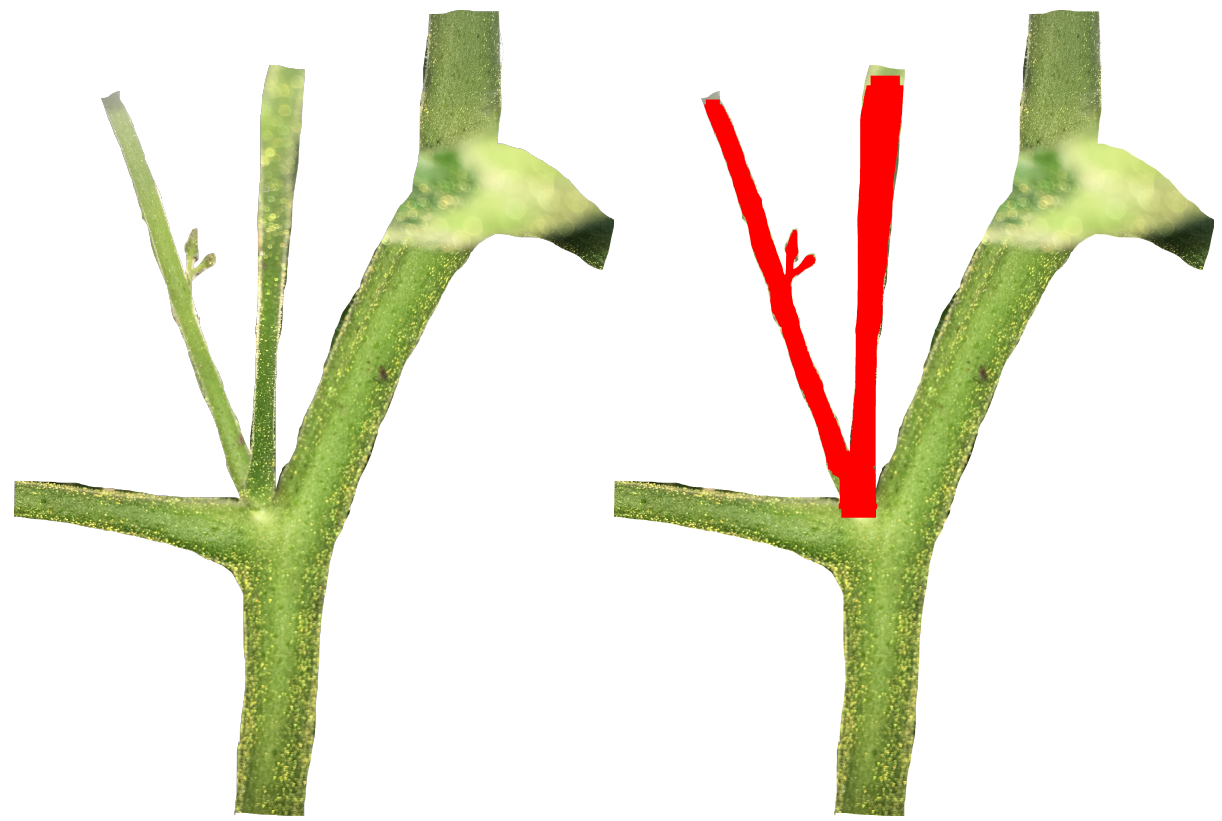

Figure 6.4: Multiple suckers spawning from same point 


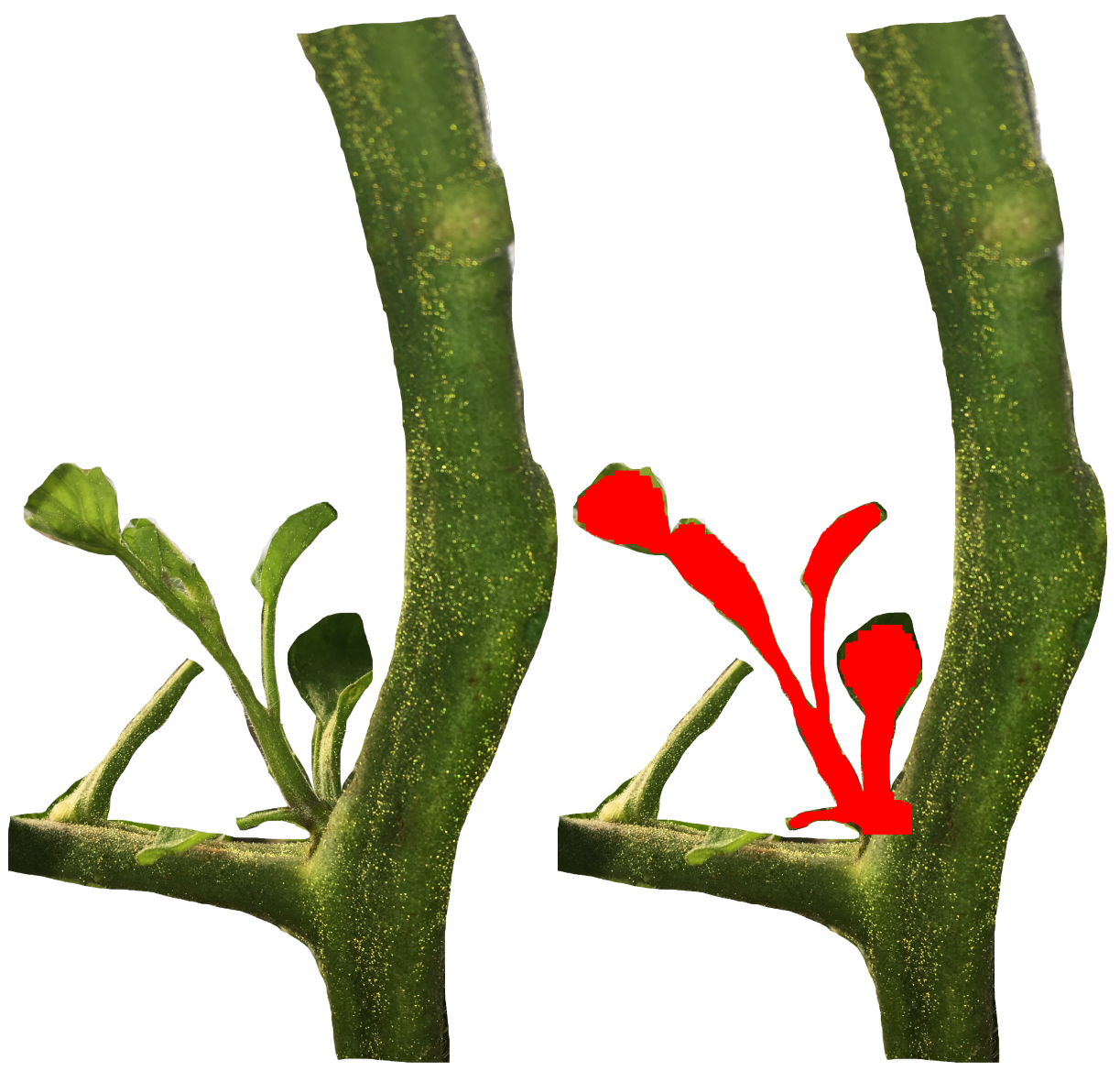

Figure 6.5: Multiple suckers spawning from same point

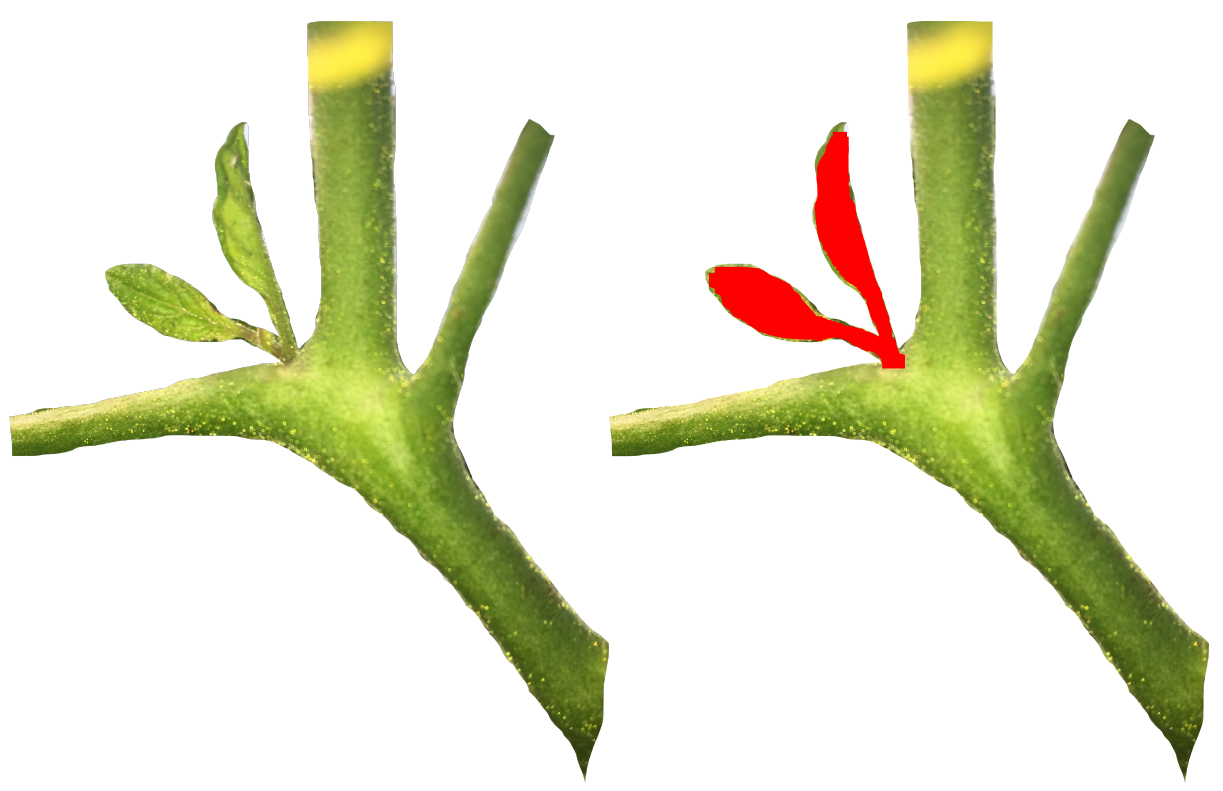

Figure 6.6: Multiple suckers spawning from same point 


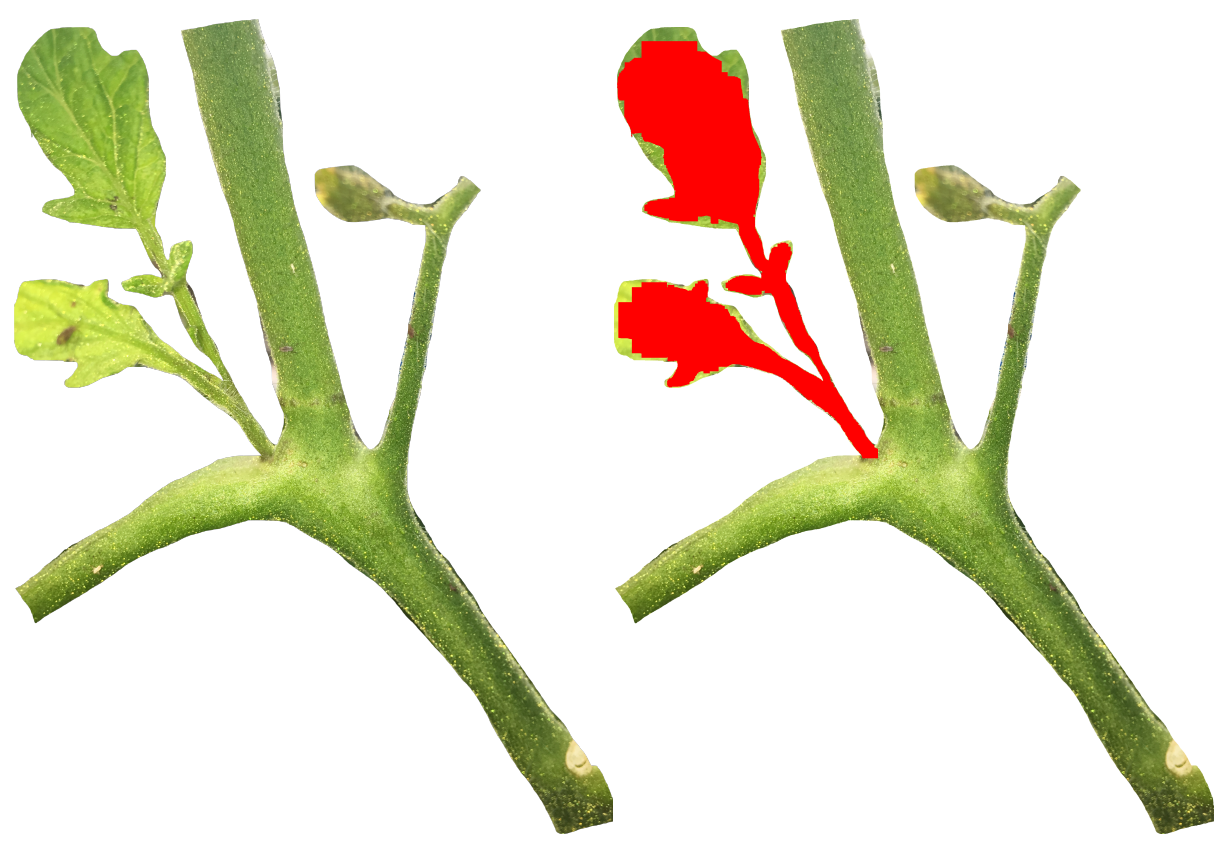

Figure 6.7: Multiple suckers spawning from same point

Example of two suckers in different locations is shown in Figures 6.8

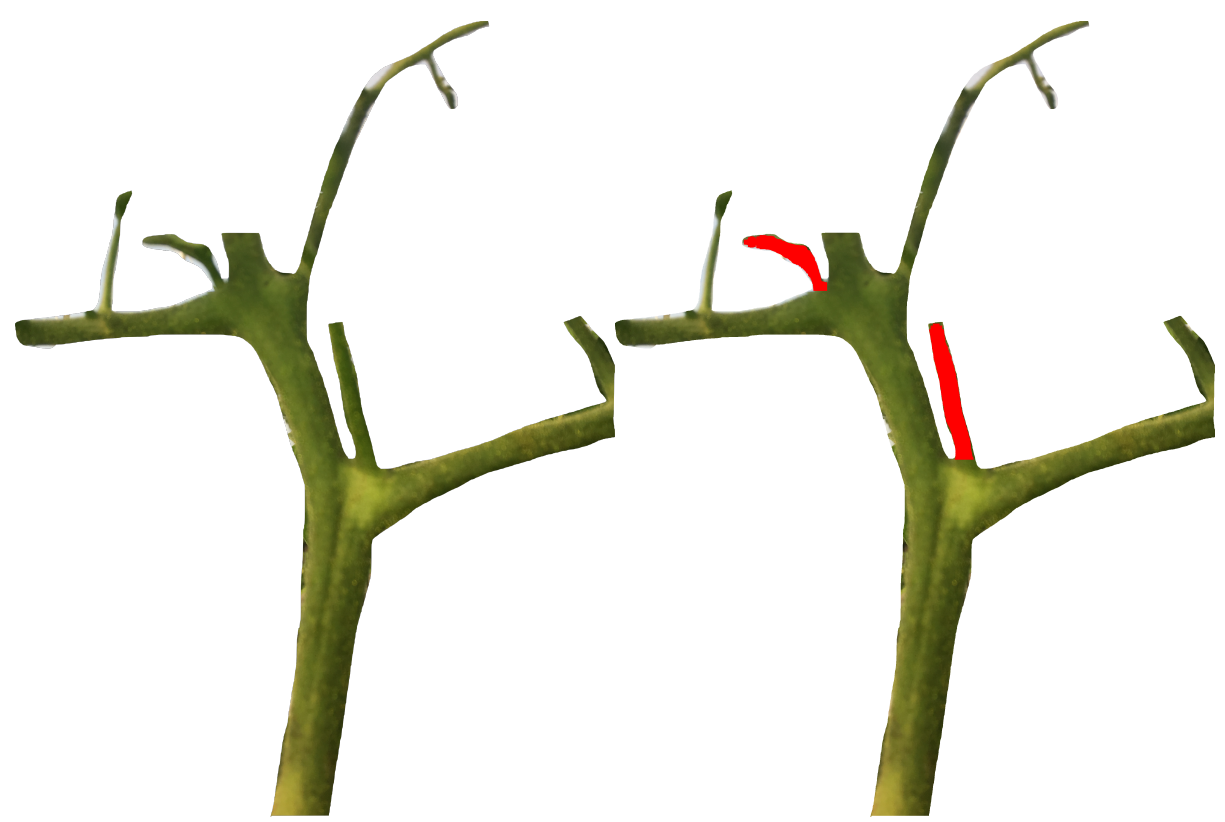

Figure 6.8: Two suckers identified in an image

Figure 6.9 is an example of two sucker locations as well as multiple suckers spawning in the same location. 


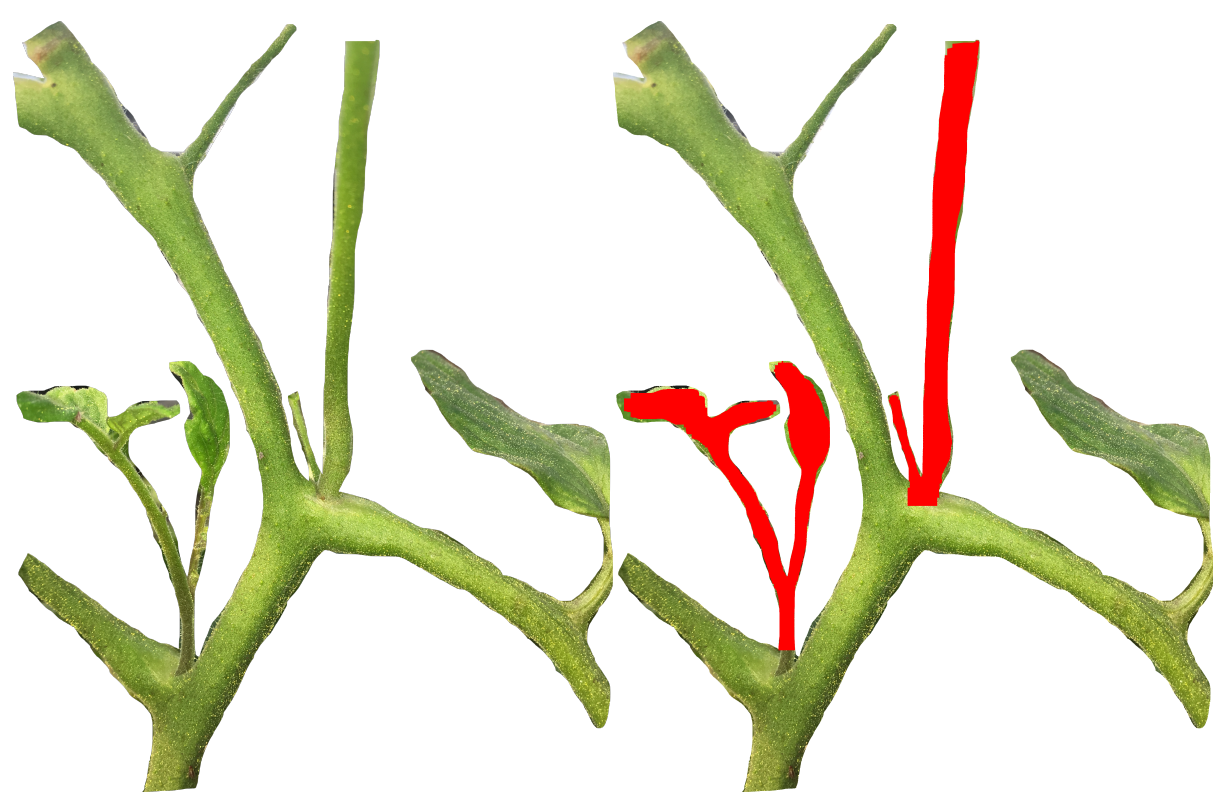

Figure 6.9: Two suckers identified in an image

Complex plant structures can be seen in Figures 6.10, 6.11, 6.12, and 6.13. 


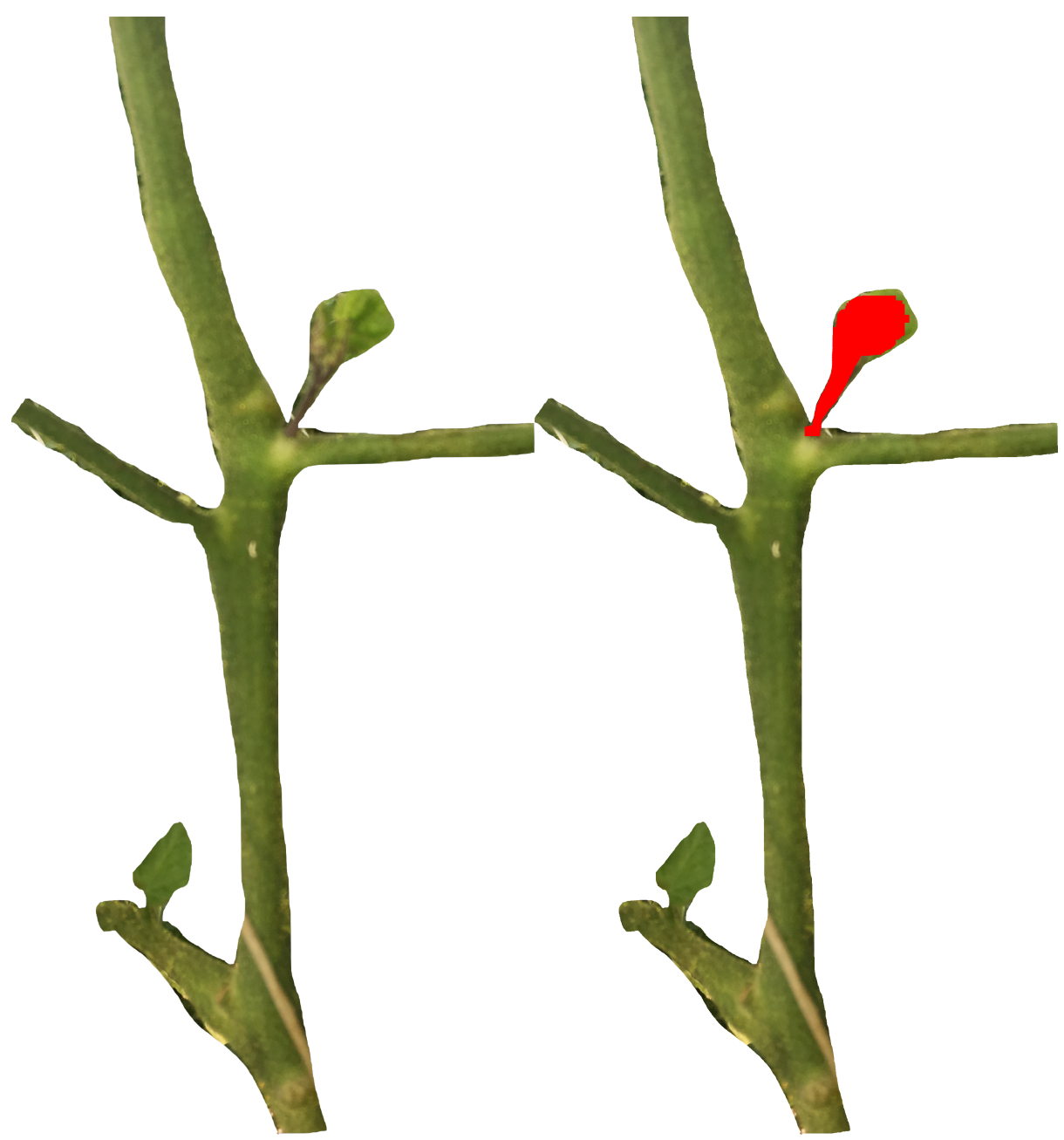

Figure 6.10: Complex plant structure

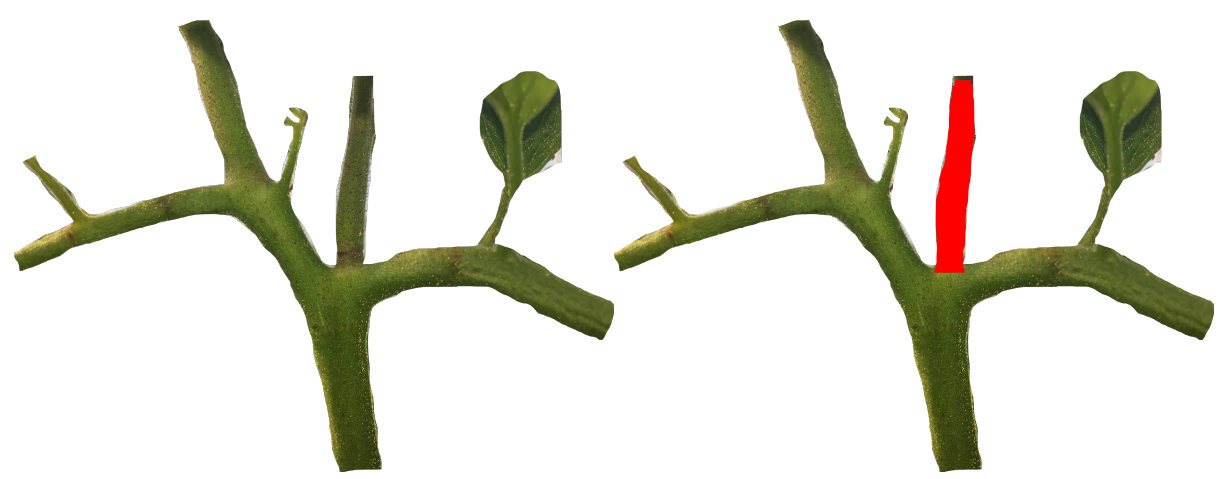

Figure 6.11: Complex plant structure 


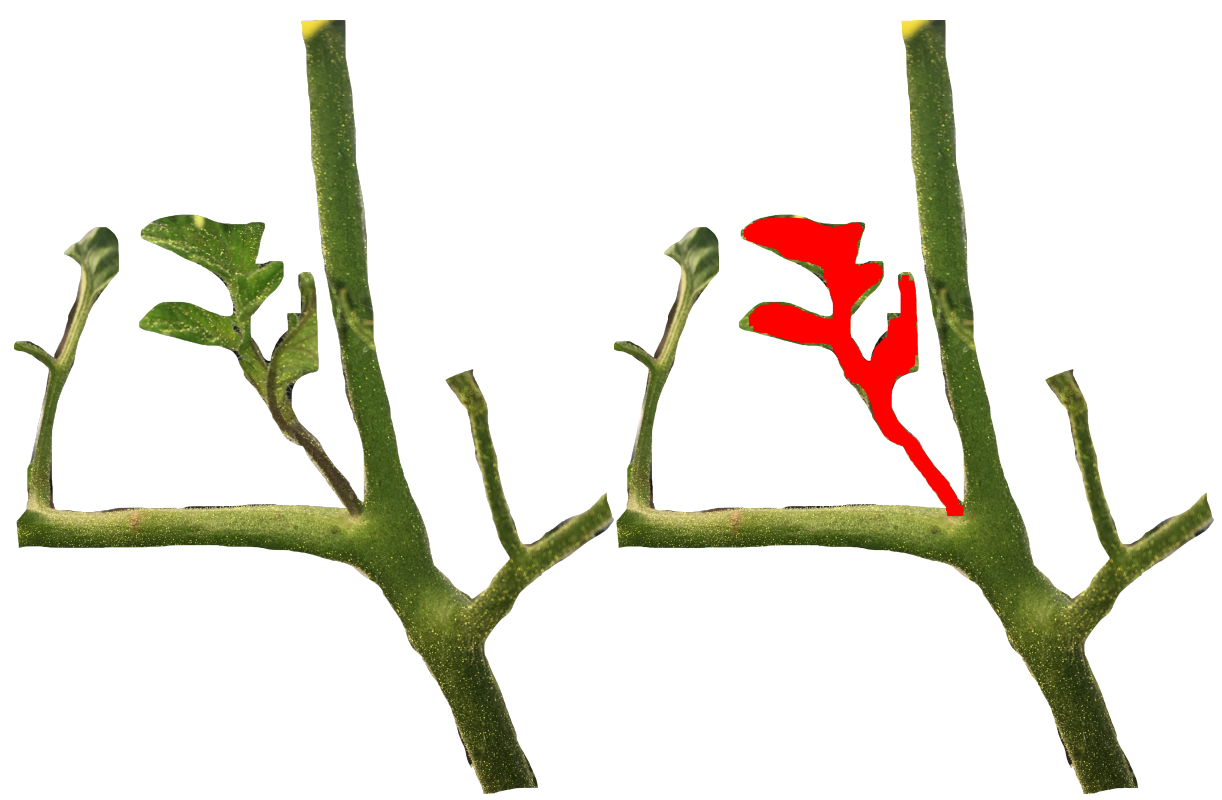

Figure 6.12: Complex plant structure with leaf sucker

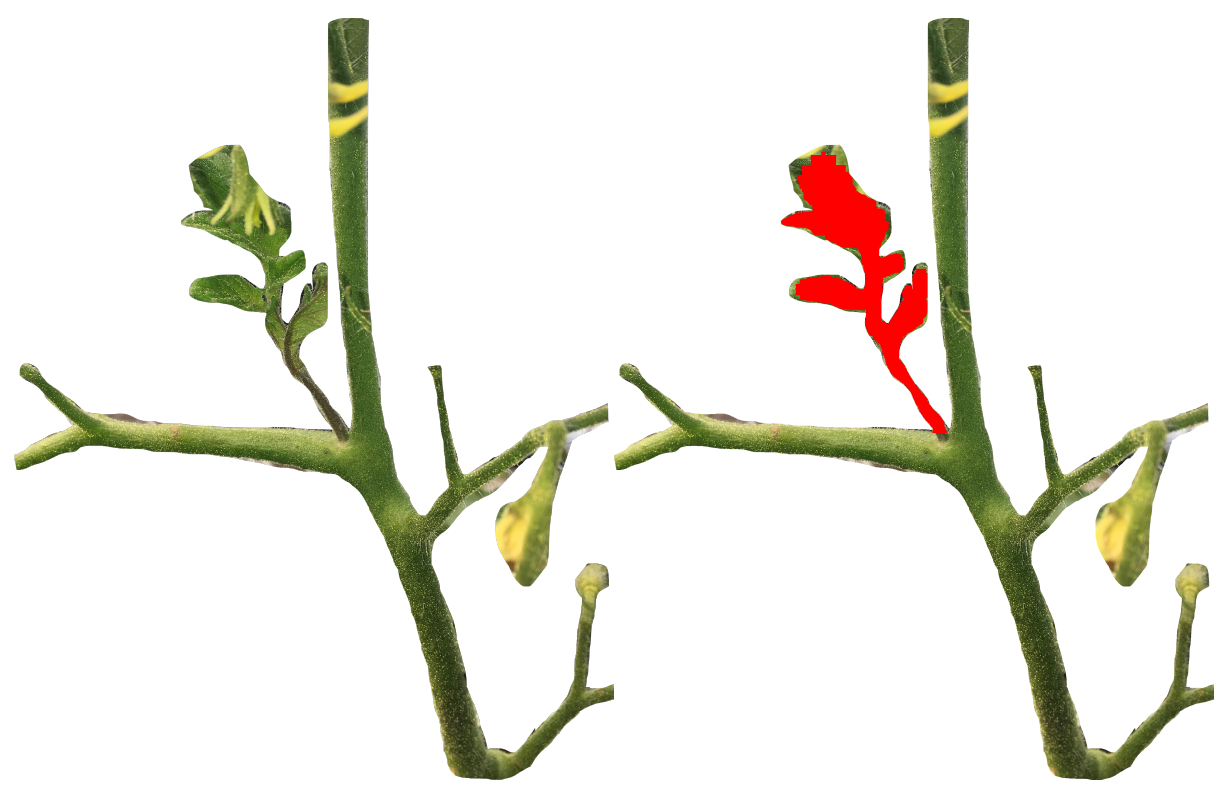

Figure 6.13: Complex plant structure with leaf sucker

Figures $6.14,6.15,6.16$, and 6.5 are close up images where the suckers are relatively close in thickness to the main stem and supporting branches 


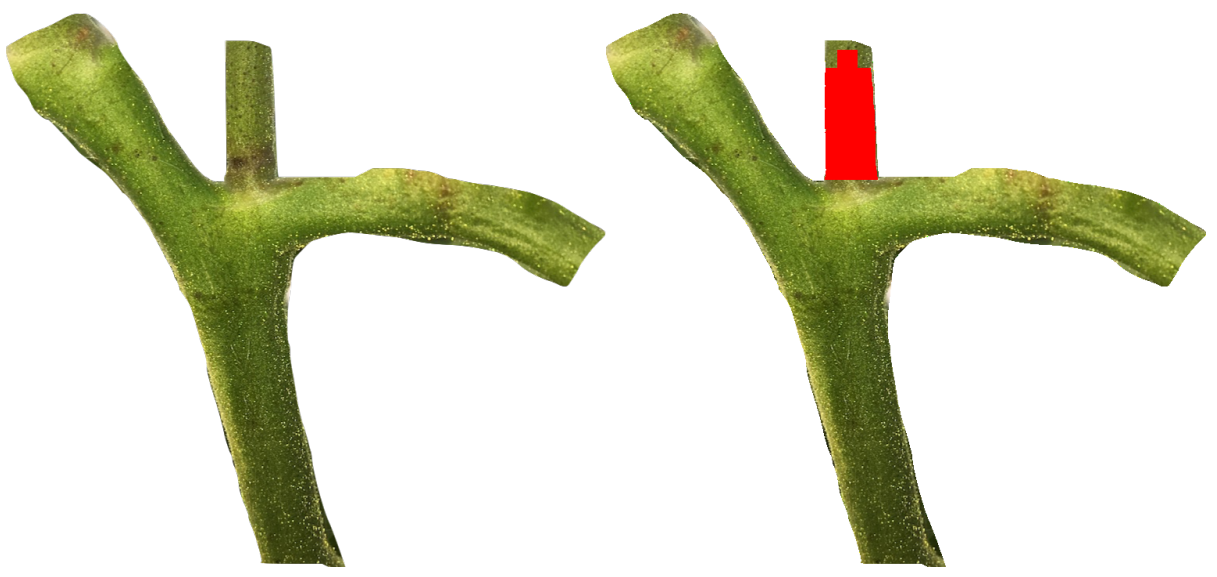

Figure 6.14: Wide sucker

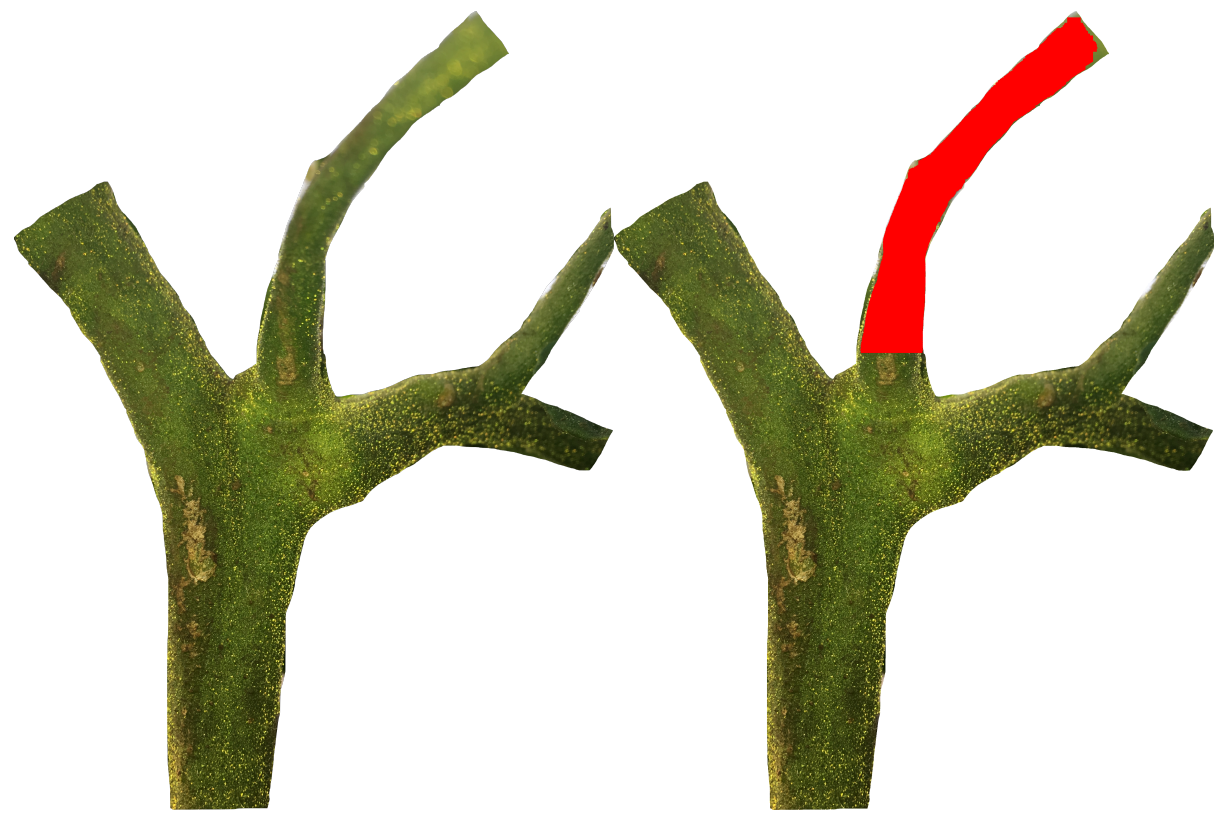

Figure 6.15: Wide sucker 


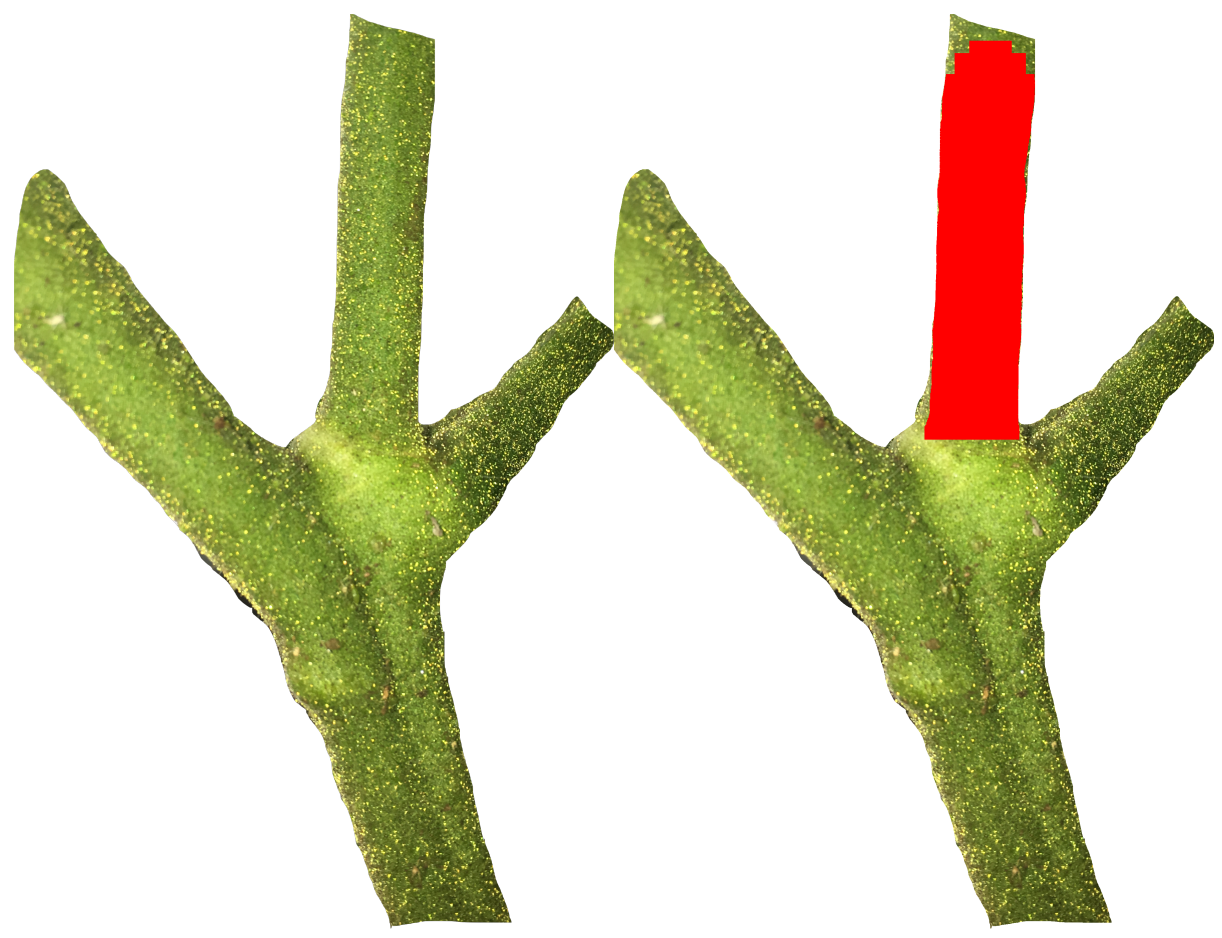

Figure 6.16: Wide sucker

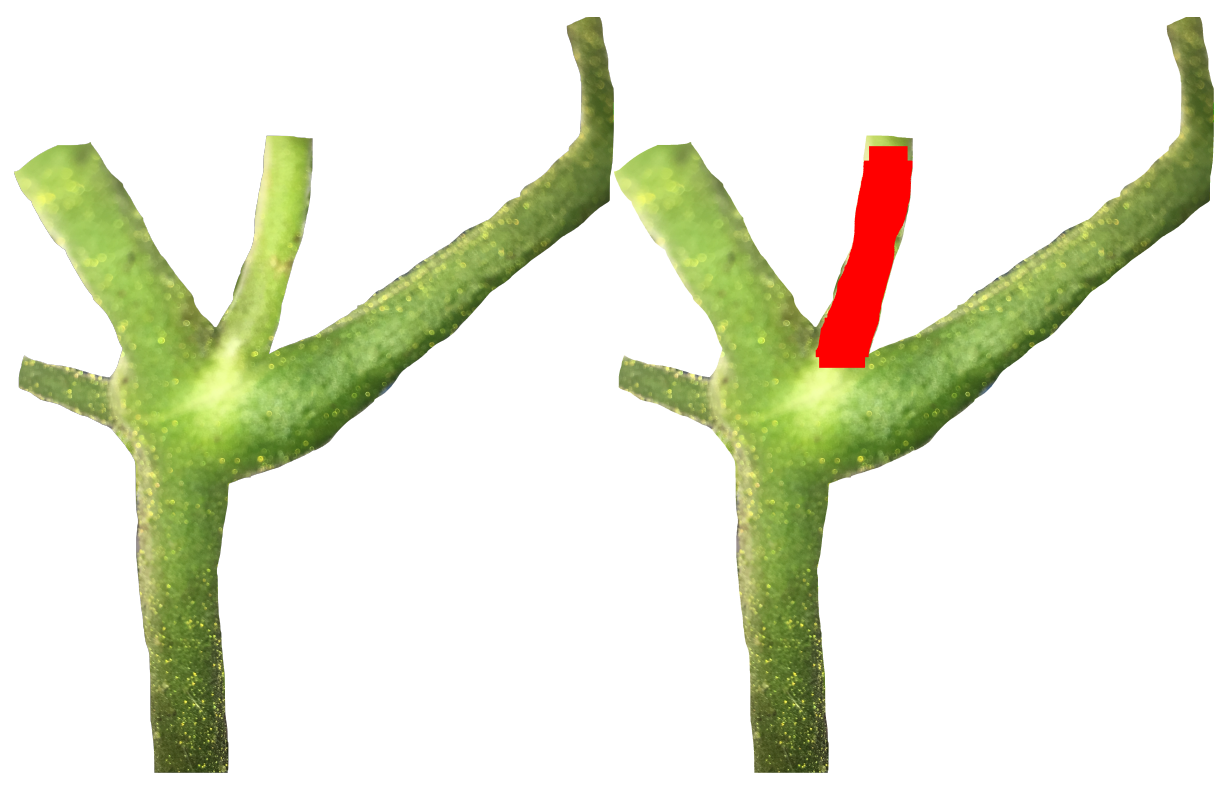

Figure 6.17: Wide sucker

Leaf suckers are correctly marked in Figures 6.18, 6.19, 6.20, and 6.21. 


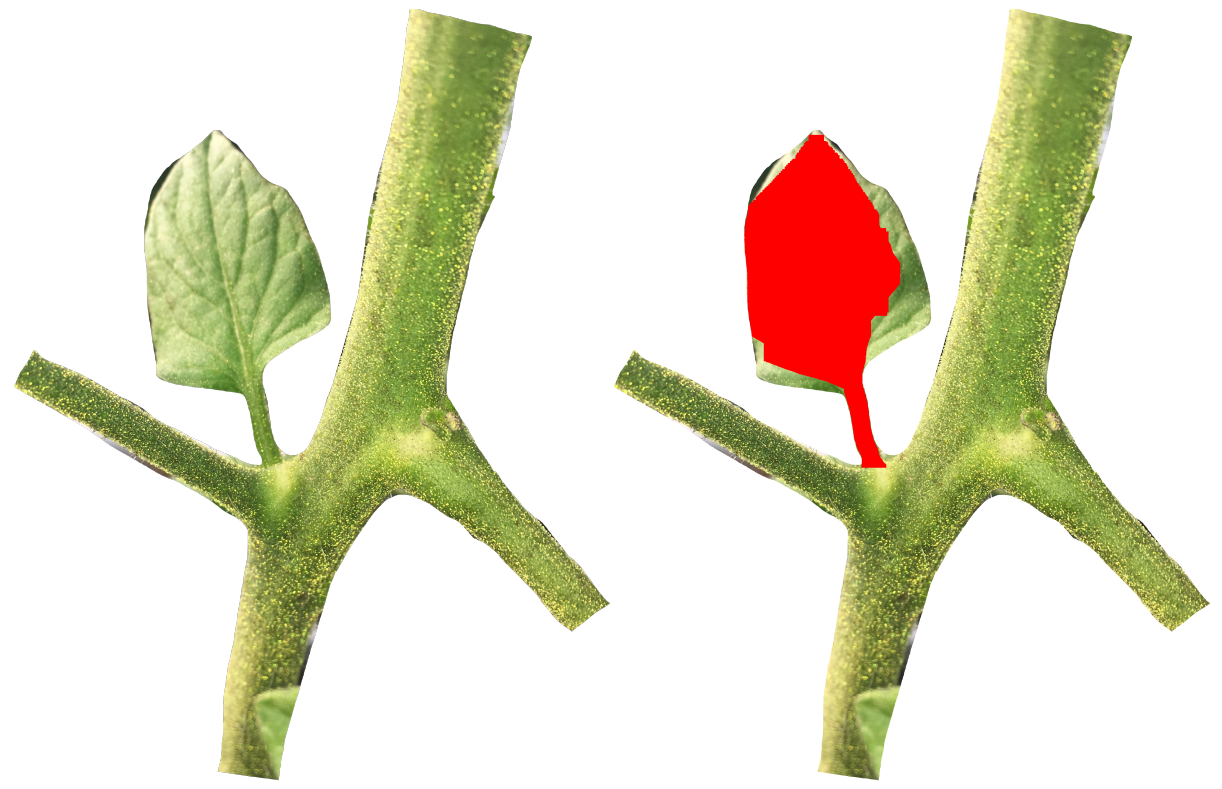

Figure 6.18: Leaf sucker

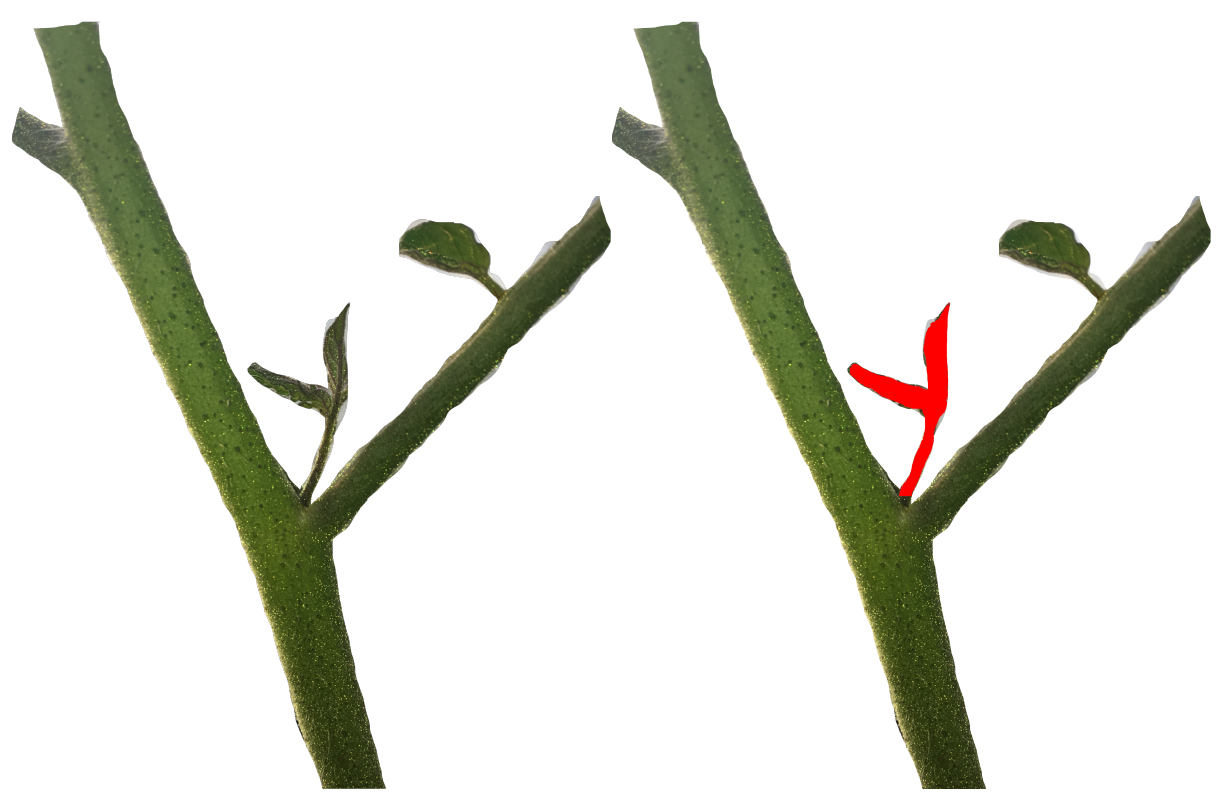

Figure 6.19: Leaf sucker 


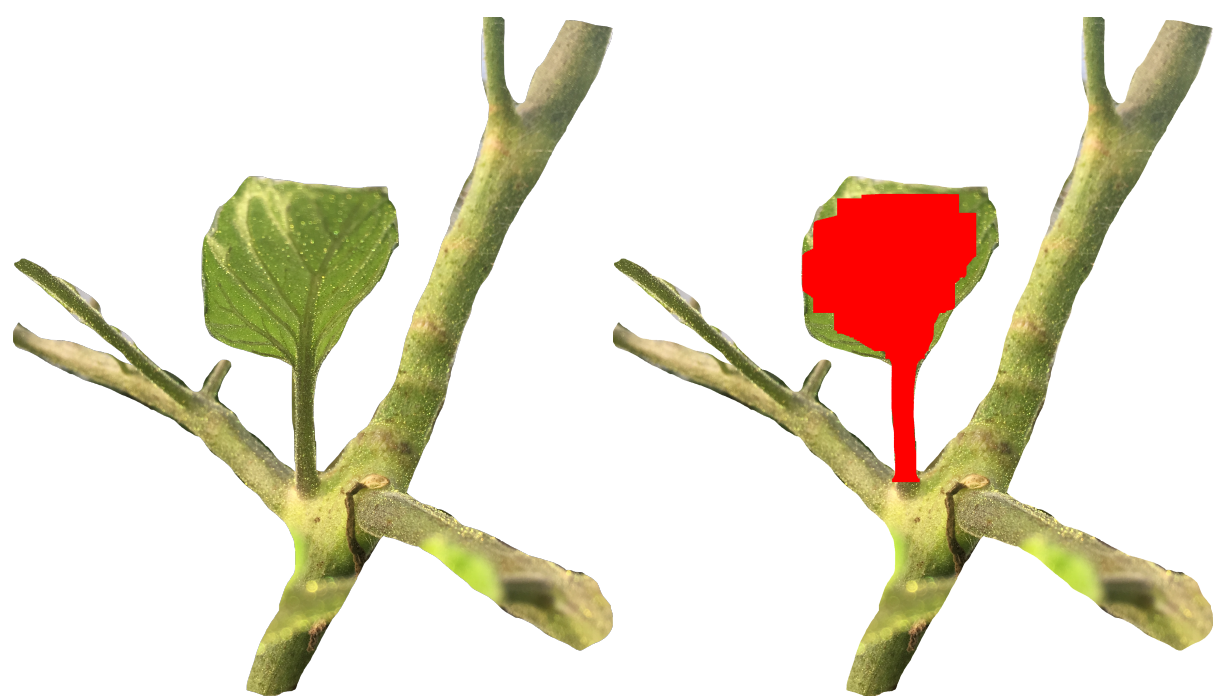

Figure 6.20: Leaf sucker with complex plant structure

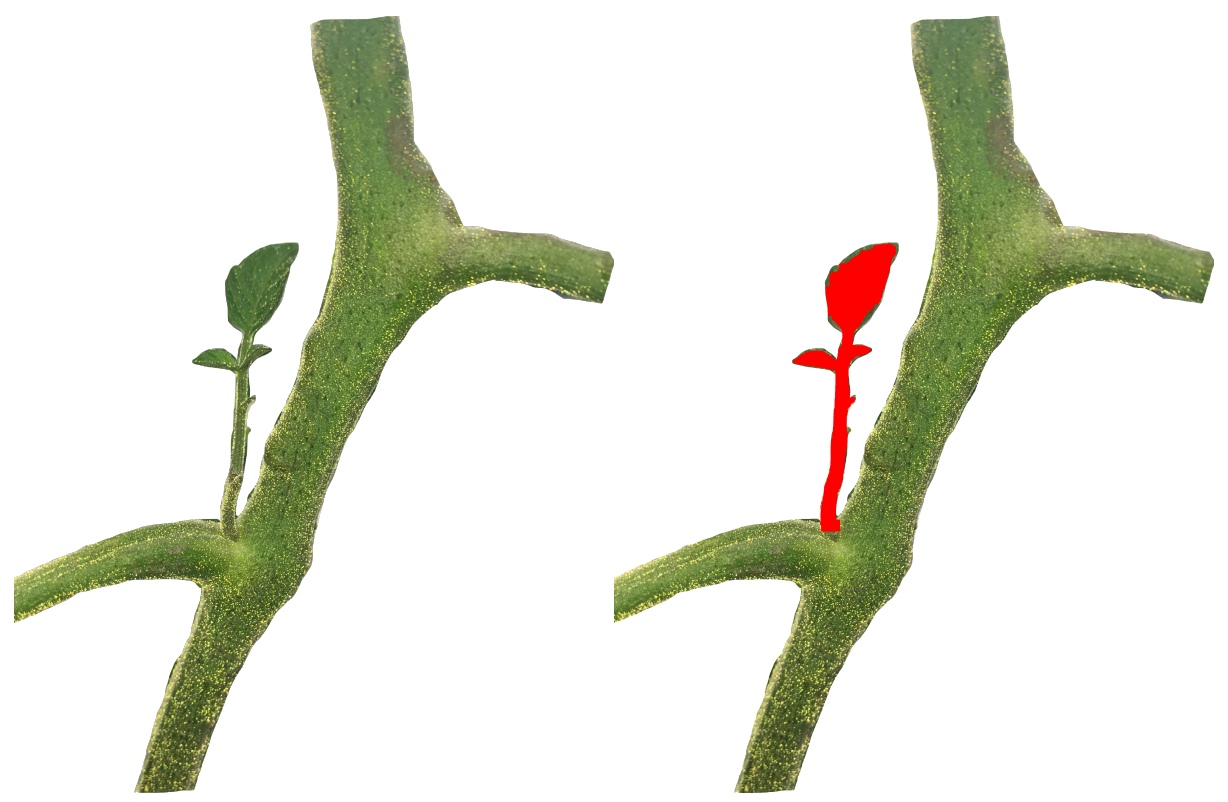

Figure 6.21: Leaf sucker

Figures $6.22,6.23$, and 6.24 have thin suckers identified. 


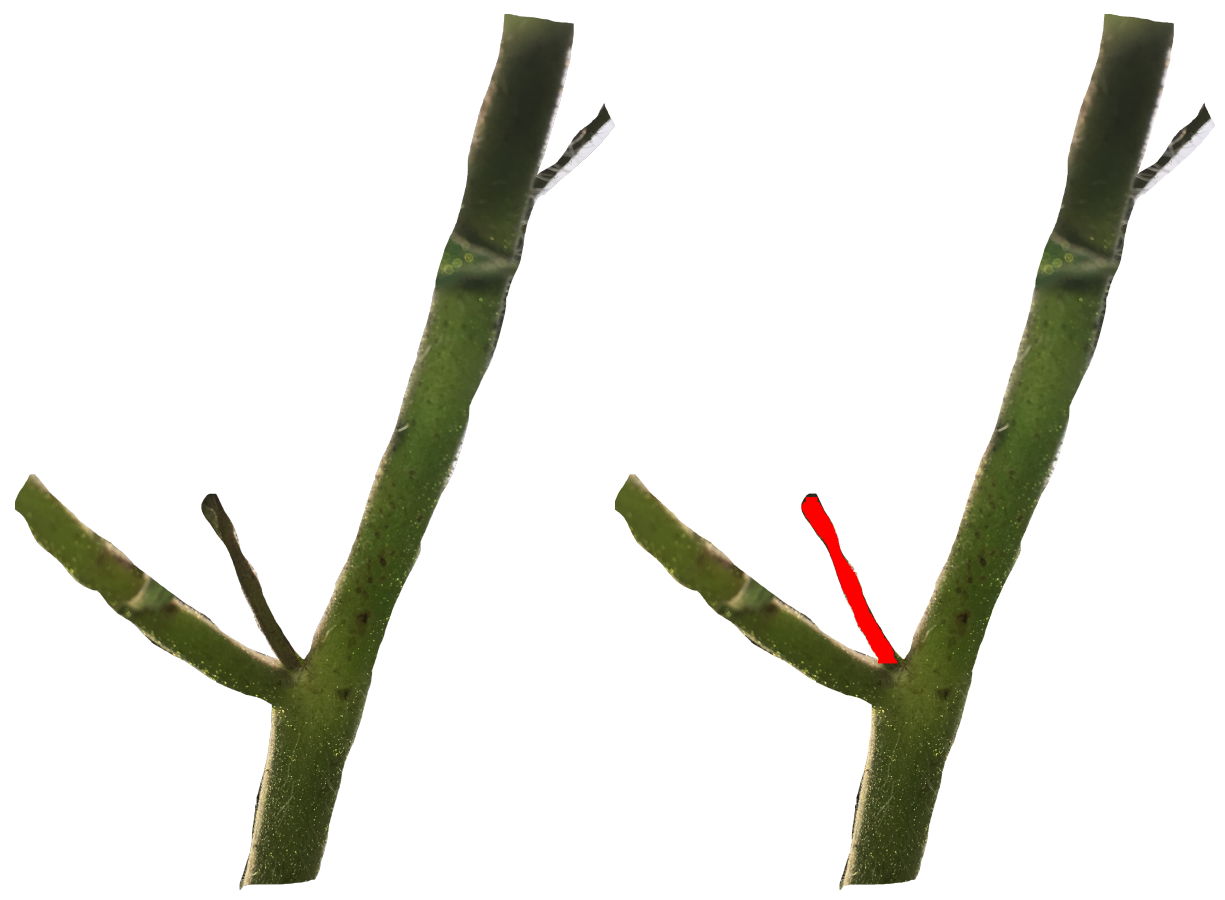

Figure 6.22: Thin sucker
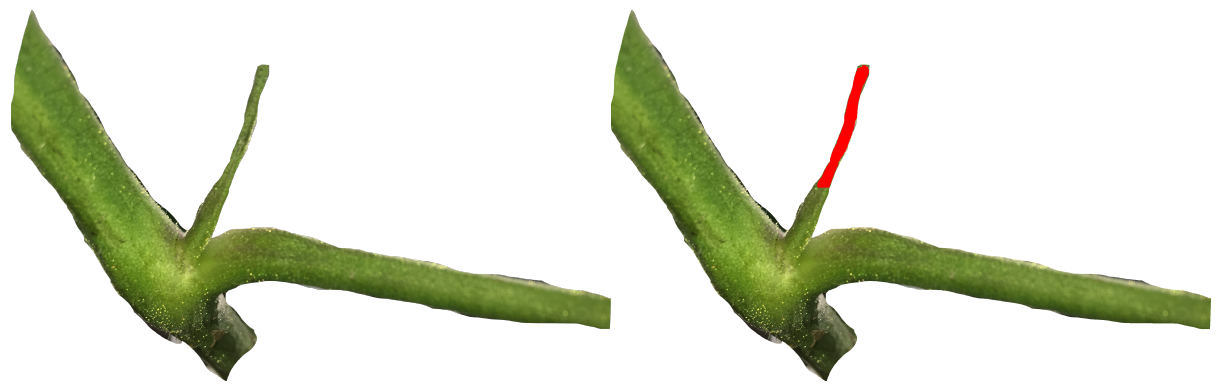

Figure 6.23: Thin sucker 


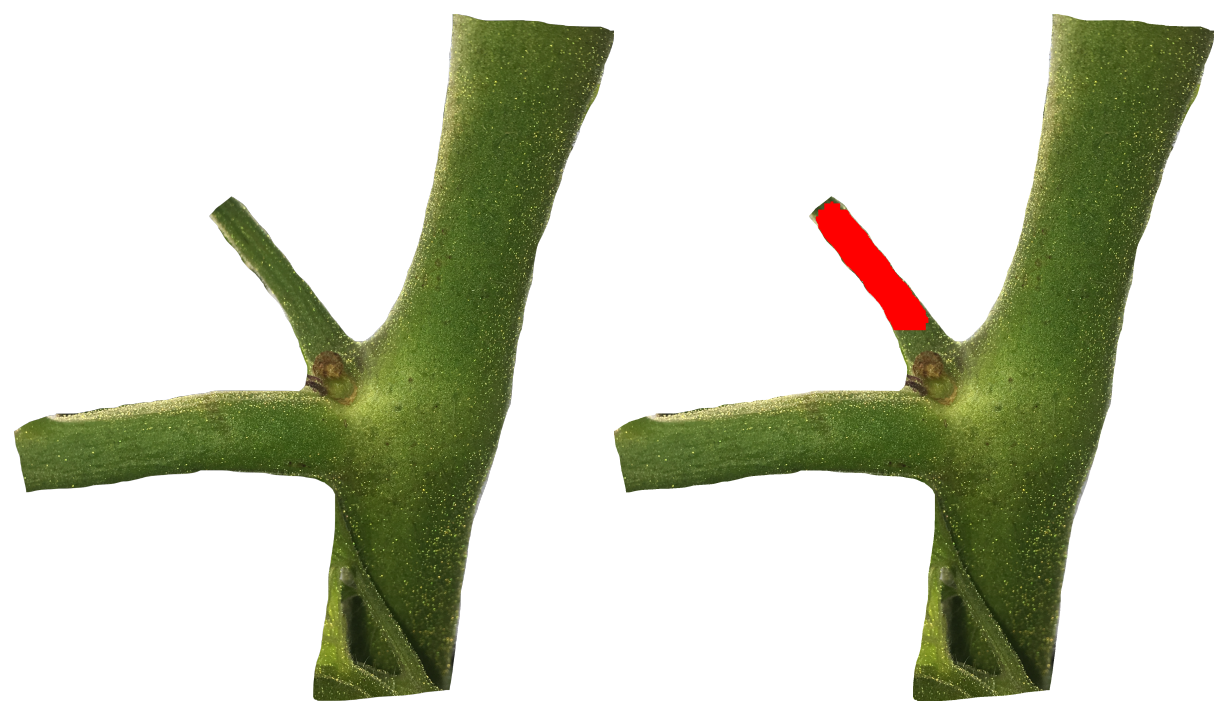

Figure 6.24: Thin sucker

\subsubsection{Downsampled Test Dataset}

Each of the 100 images were downsampled 9 times: $10 \%$ to $90 \%$ of the original size. These images were run to test the scale invariance of the method developed in this thesis. Since the sole purpose is to test the scale invariance, we only analyze the 97 images that passed in the original data set. Upon running the sucker detection algorithm on the downsampled images, we noticed two distinct causes for differences in output from the original 100 image dataset: skeletonization loops, failure to meet thresholds for BSOI, sucker validation, etc.

Skeletonization errors occur when SciKit's skeletonization algorithm generates a much different skeleton than the original image. The difference in skeleton is expected to vary slightly with the downsampled images, but the introduction of new BSs connecting previously disconnected BSs is what causes the error. Specifically, loops between BSs are created, which causes the recursive prune algorithm to prune more BSs than necessary. Since the pruning algorithm is recursive and prunes every branch that is touching the sucker BS, it is possible for an unintended BS to be pruned. This scenario is shown in Figure 6.25. 


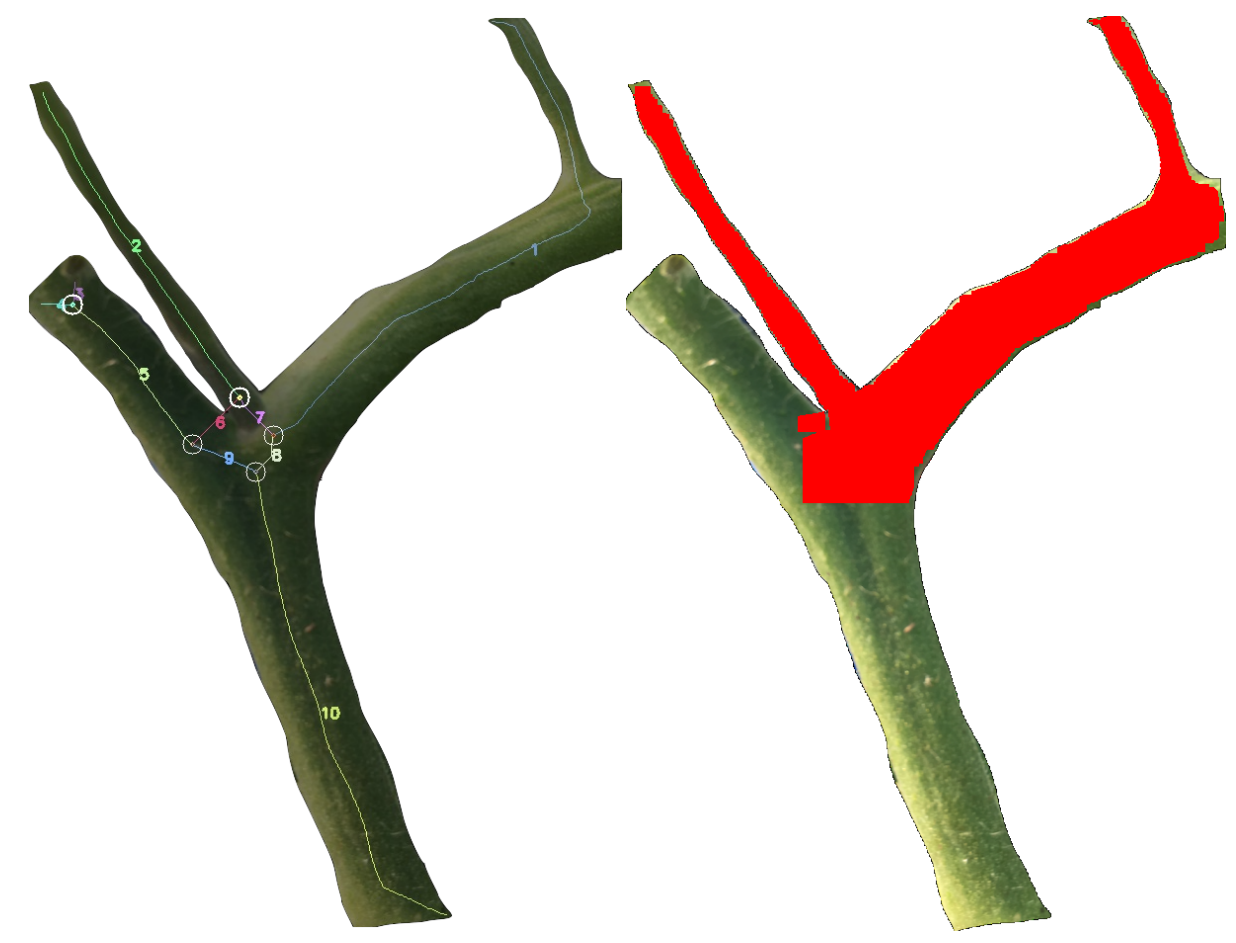

Figure 6.25: Skeletonization error: visible loop not representative of the structure

A threshold failure is a direct failure of the algorithm. It is a failure of the algorithm to identify a BS as a BSOI, or identifying a BS as a sucker when it should not be. This is the same error as experienced by Figure 6.3.

Each image that passed in the original dataset was tested 9 times, each with a donwsampling rate of $10 \%$ decrements. Since 97 images passed in the original dataset, this equates to 873 downsampled test images. The total passing rates and errors for the 873 downsampled images are covered in Table 6.2. Ideally, all 873 downsampled images would yield the same result as the original image. Similar to the original dataset, we achieved a high percentage for correct images when all 873 images are taken into account. We observed that only some of the downsampling images failed for a particular original image. Table 6.2 treats each downsampled image separately. On the other hand, Table 6.3, treats a failure for one of the downsampled images, as a failure for the entire downsampled set. With this method of measuring 
success, we only achieved a success rate of $75.3 \%$. But excluding skeletonization errors from the total, which we have no control over in our current algorithm, our success rate jumps to $87.9 \%$. Table 6.4 shows the different downsampling rates and the corresponding errors that occurred. In general, the lower downsampling rates exhibited more skeletonization errors. This is most likely due to the interpolation that occurs in the downsampling process. However, there is no distinct correlation between downsampling rate and algorithm errors.

Table 6.2: Accuracy for the 873 downsampled images

\begin{tabular}{|c|c|c|}
\hline Image Result & Occurrences & Percent of Total (873) \\
\hline Skeletonization Error & 34 & $4.0 \%$ \\
\hline Threshold Error & 29 & $3.3 \%$ \\
\hline Correct & 810 & $92.8 \%$ \\
\hline
\end{tabular}

Table 6.3: Accuracy for the 97 unique images

\begin{tabular}{|c|c|c|}
\hline Image Result & Occurrences & Percent of Total (97) \\
\hline Skeletonization Error & 14 & $14.4 \%$ \\
\hline Threshold Error & 10 & $10.3 \%$ \\
\hline Correct & 73 & $75.3 \%$ \\
\hline
\end{tabular}


Table 6.4: Errors by downsampled rate

\begin{tabular}{|c|c|c|}
\hline Downsampled Rate & Skeletonization Error Occurrences & Threshold Error Occurrences \\
\hline $10 \%$ & 7 & 5 \\
\hline $20 \%$ & 7 & 3 \\
\hline $30 \%$ & 2 & 2 \\
\hline $40 \%$ & 4 & 4 \\
\hline $50 \%$ & 4 & 2 \\
\hline $60 \%$ & 3 & 1 \\
\hline $70 \%$ & 3 & 6 \\
\hline $80 \%$ & 3 & 3 \\
\hline $90 \%$ & 1 & 3 \\
\hline
\end{tabular}

\subsection{Marking Endpoint of Sucker}

Another metric measured was whether or not the endpoint of a sucker was properly marked. As mentioned in subsection 5.6.2, the algorithm determined an endpoint for which it stopped marking red. The original 100 images were visually inspected and each sucker identified was given a pass or fail depending on where the endpoint of the sucker was marked. Figure 6.26 exemplifies the two cases where a sucker could fail marking the endpoint. An endpoint marked below the ideal position is said to be low, while an endpoint marked above is considered as high. With a $79.7 \%$ success rate, the accuracy of the endpoint selection was much lower than that of the sucker identification. This is mostly because a very simple, heuristic approach was used to select the endpoint. 
Table 6.5: Marking endpoint accuracy

\begin{tabular}{|c|c|c|}
\hline Endpoint Marking Type & Occurrences & Percent of Total Endpoints (118) \\
\hline Low & 12 & $10.2 \%$ \\
\hline High & 12 & $10.2 \%$ \\
\hline Correct & 94 & $79.7 \%$ \\
\hline
\end{tabular}

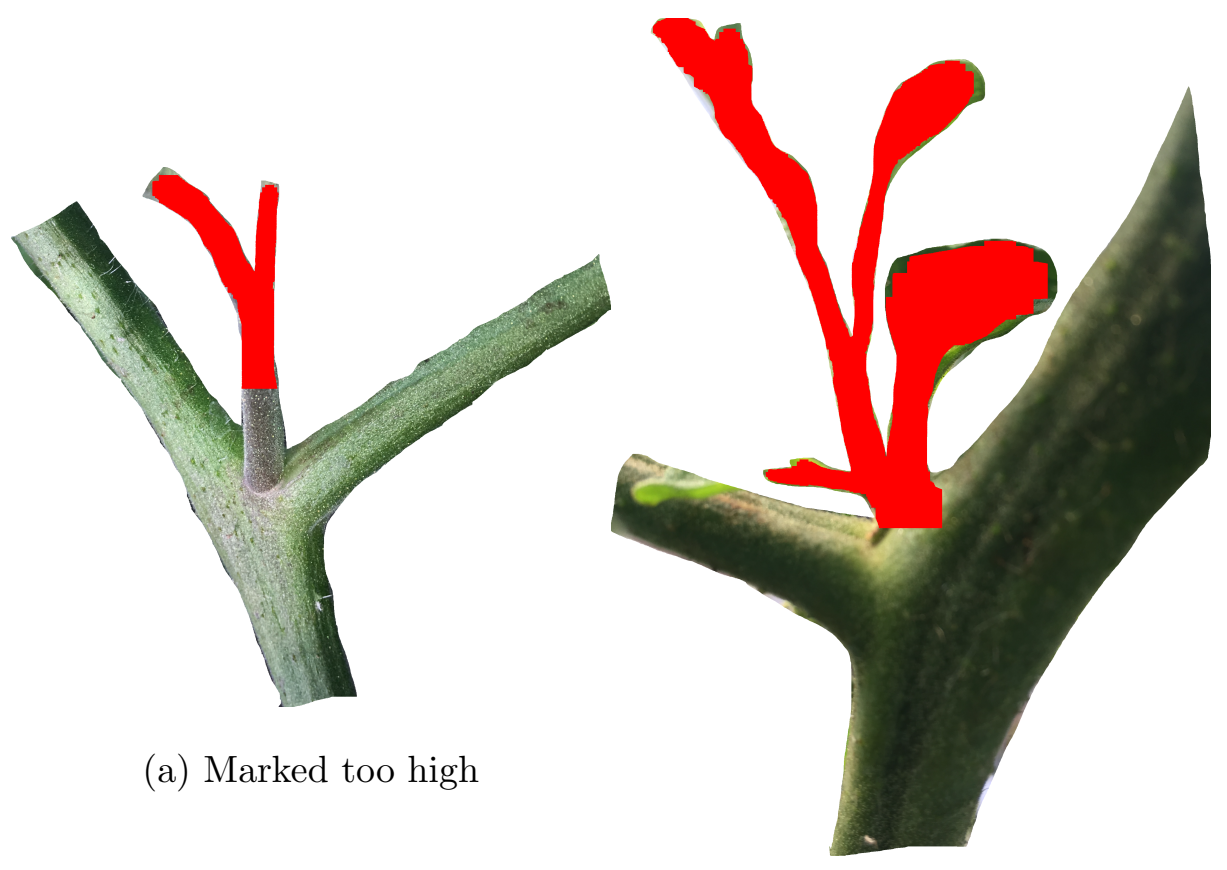

(b) Marked too low

Figure 6.26: Both cases of marking failure

\subsection{Timing}

The timing and efficiency for the 100 original sample images is listed in Table 6.6. The table is split up into the four major components of this project: preprocessing, skeleton classifying, intersect statistics, and finding the suckers. The components developed in this project took a much shorter time to complete than the preprocessing step, which was developed by OpenCV and SciKit. The entire sucker identification al- 
gorithm resulted in an impressive $6.5 \mathrm{MP}$ per second, making this algorithm practical for real world application.

Table 6.6: Timing for the 100 original test images

\begin{tabular}{|c|c|c|c|c|c|}
\hline & Preprocessing & Classifying & Stats & Find Suckers & Total \\
\hline Time (s) & 1969.4 & 137.5 & 0.6 & 40.2 & 2147.8 \\
\hline \% of Total Time & $91.7 \%$ & $6.4 \%$ & $0.03 \%$ & $1.9 \%$ & $100 \%$ \\
\hline M Pixels / sec & 0.6 & 8.5 & 1817.8 & 28.9 & 6.5 \\
\hline
\end{tabular}




\section{Chapter 7}

\section{CONCLUSIONS}

The sucker detection algorithm developed in this thesis proved to be effective and, for the most part, scale invariant. Operating with only 2-dimensional images was challenging, but a high rate of success was still achieved for the sucker detection component. The processing speed for the algorithm allows this to be a practical solution for a real world application. The novel skeleton classification method developed in this thesis can be extended so a multitude of applications that use skeleton images.

\subsection{Algorithm Effectiveness}

We were able to successfully identify about $97 \%$ of the suckers in the test images, however, there were some crucial assumptions. The algorithm starts by crawling up the bottom most branch. This creates a dependency that might not be realistic in a

real world, automated system. Also, the endpoint marking portion of the algorithm was less successful, with only a $79 \%$ success rate. Fortunately, this component of the algorithm is less important than the actual identification of sucker BSs. The downsampling results were not as successful as the original data set, but they were still promising at about $75 \%$ and $90 \%$ with and without skeletonization errors respectively.

\subsection{2-Dimensional Images}

The algorithm created in this thesis operates in the 2-dimensional space due to the lack of depth sensors. Despite not having depth information on the images, clear physical characteristics of suckers were discovered and utilized to achieve a high detection rate. 


\subsection{Processing Speed}

The high resolution images were processed with impressive speed. Ideally, images of such high resolution may not be necessary and quicker processing times can be witnessed with the use of lower resolution images. The most time-consuming part of the

algorithm stemmed from OpenCV and SciKit's image functions in the preprocessing step. The skeleton classification and sucker detection algorithms were relatively fast when compared to the preprocessing steps.

\subsection{Skeleton Classification}

The novel method developed to classify a skeleton image proved to be fast, efficient, and effective. The process of classifying is extremely fast because of numpy's and OpenCV's optimized methods. By removing the pixels that make up the IPs in a skeleton, the OpenCV connectedComponents method can be utilized to quickly label all the BSs. This method can be applied in other skeleton applications to separate the different BSs and IPs of a skeleton image efficiently and accurately. 
Chapter 8

\section{FUTURE WORK}

This thesis is meant to serve as the first step towards an autonomous tomato pruning system, but there is much work to be done for this to be achieved: the endpoint marking needs to be improved, depth must be integrated into the sucker detection algorithm, and the sucker characteristics can have a non-heuristic approach to improve accuracy.

\subsection{Improved Endpoint Marking}

A simple thresholding technique was used for determining the endpoint of a sucker. This yielded only an $80 \%$ success rate. An improved method for determining the endpoint can result in more accurate markings of suckers.

\subsection{3-Dimensional Point Cloud}

In a real-world application, depth within the images will have to be taken into account to filter out the background pixels and accurately prune the identified sucker. An interesting project would be to modify the algorithm developed in this thesis to make it capable of inputting a depth or 3-D image. Figure 6.1 can possibly be remedied if

depth sensors are used to construct a 3-D point cloud of the structure of the plant. The use of a depth sensor, such as a stereo vision camera, can potentially mitigate some of the skeletonization loops and errors that occurred. 


\subsection{Filtering Background Pixels}

Background pixels were manually excluded from the test images in this thesis, but an algorithm for filtering out the background pixels would have to be implemented to make the sucker identification algorithm useful. Some possibilities for doing this were discussed in the literature review, where lasers or stereo cameras were used to gain a depth aspect of an image.

\subsection{Non-Heuristic Approach for sucker characteristics}

A neural network trained with different BSOIs would provide a more accurate and error tolerant result than the thresholds used in this project for classifying a BSOI. The test data set used in this thesis can be leveraged to build and train a neural network. A neural network could potentially solve the decrease in accuracy with downsampling.

\subsection{Full Scale Autonomous Pruning System}

This thesis is meant to serve as an initial step towards the invention of a full scale, autonomous pruning system.

\subsubsection{Greenhouse Preparations}

A rail system could possibly be installed in the greenhouse to make navigation an easier task. Rather than guide the robot freely through the greenhouse, a railed system would enable a set, known path for the navigation algorithm. 


\subsubsection{Algorithms}

The onboard computer is loaded with various algorithms to perform the necessary tasks. Each algorithm is responsible for a key part of the pruning process: navigating the robot through the aisles, tomato plant survey and inspection, and sucker identification. Ideally, the algorithm developed in this thesis will be used as the basis for the sucker identification algorithm. 


\section{BIBLIOGRAPHY}

[1] Cal Poly Github. http://www.github.com/CalPoly.

[2] The daily garden.

[3] Numpy.

[4] Opencv library.

[5] Scikit-image.

[6] S. A. Akbar, N. M. Elfiky, and A. Kak. A novel framework for modeling dormant apple trees using single depth image for robotic pruning application. In Robotics and Automation (ICRA), 2016 IEEE International Conference on, pages 5136-5142. IEEE, 2016.

[7] S. Amundson, D. E. Deyton, D. A. Kopsell, W. Hitch, A. Moore, and C. E. Sams. Optimizing plant density and production systems to maximize yield of greenhouse-grown trusttomatoes. HortTechnology, 22(1):44-48, 2012.

[8] J. Chen and G. Lal. Pruning and staking tomatoes. AVRDC International Cooperators Guide, AVRDC pub, pages 9-490, 1999.

[9] J. M. Davis and E. A. Estes. Spacing and pruning affect growth, yield, and economic returns of staked fresh-market tomatoes. Journal of the American Society for Horticultural Science, 118(6):719-725, 1993.

[10] . DeJohn. Simple tomato pruning technique — gardener's supply company blog, Jun 2013.

[11] N. M. Elfiky, S. A. Akbar, J. Sun, J. Park, and A. Kak. Automation of dormant pruning in specialty crop production: An adaptive framework for 
automatic reconstruction and modeling of apple trees. In Computer Vision and Pattern Recognition Workshops (CVPRW), 2015 IEEE Conference on, pages 65-73. IEEE, 2015.

[12] M. Gao and T.-F. Lu. Image processing and analysis for autonomous grapevine pruning. In Mechatronics and Automation, Proceedings of the 2006 IEEE International Conference on, pages 922-927. IEEE, 2006.

[13] Josh. My garden in florida, Jan 1970.

[14] L. Kanyomeka and B. Shivute. Influence of pruning on tomato production under controlled environments. Agricultura Tropicaet Subtropica, 32(2):79-81, 2005.

[15] Martin, Kay, and Intel. Intel integrated performance primitives (intel ipp), Jan 2018.

[16] C. Mbonihankuye, P. Kusolwa, and T. Msogoya. Assessment of the effect of pruning systems on plant developmental cycle, yield and quality of selected indeterminate tomato lines. Acta Hortic (ISHS), 1007:535-542, 2013.

[17] J. McCulloch and R. Green. Detecting wires in the canopy of grapevines using neural networks: A robust and heuristic free approach. In Image and Vision Computing New Zealand (IVCNZ), 2013 28th International Conference of, pages 334-339. IEEE, 2013.

[18] H. Medeiros, D. Kim, J. Sun, H. Seshadri, S. A. Akbar, N. M. Elfiky, and J. Park. Modeling dormant fruit trees for agricultural automation. Journal of Field Robotics, 34(7):1203-1224, 2017.

[19] S. Xu, Y. Xun, T. Jia, and Q. Yang. Detection method for the buds on winter vines based on computer vision. In Computational Intelligence and Design 
(ISCID), 2014 Seventh International Symposium on, volume 2, pages 44-48.

IEEE, 2014. 


\section{APPENDICES}

Appendix A

APPENDIX A

\section{A.1 Downsample}

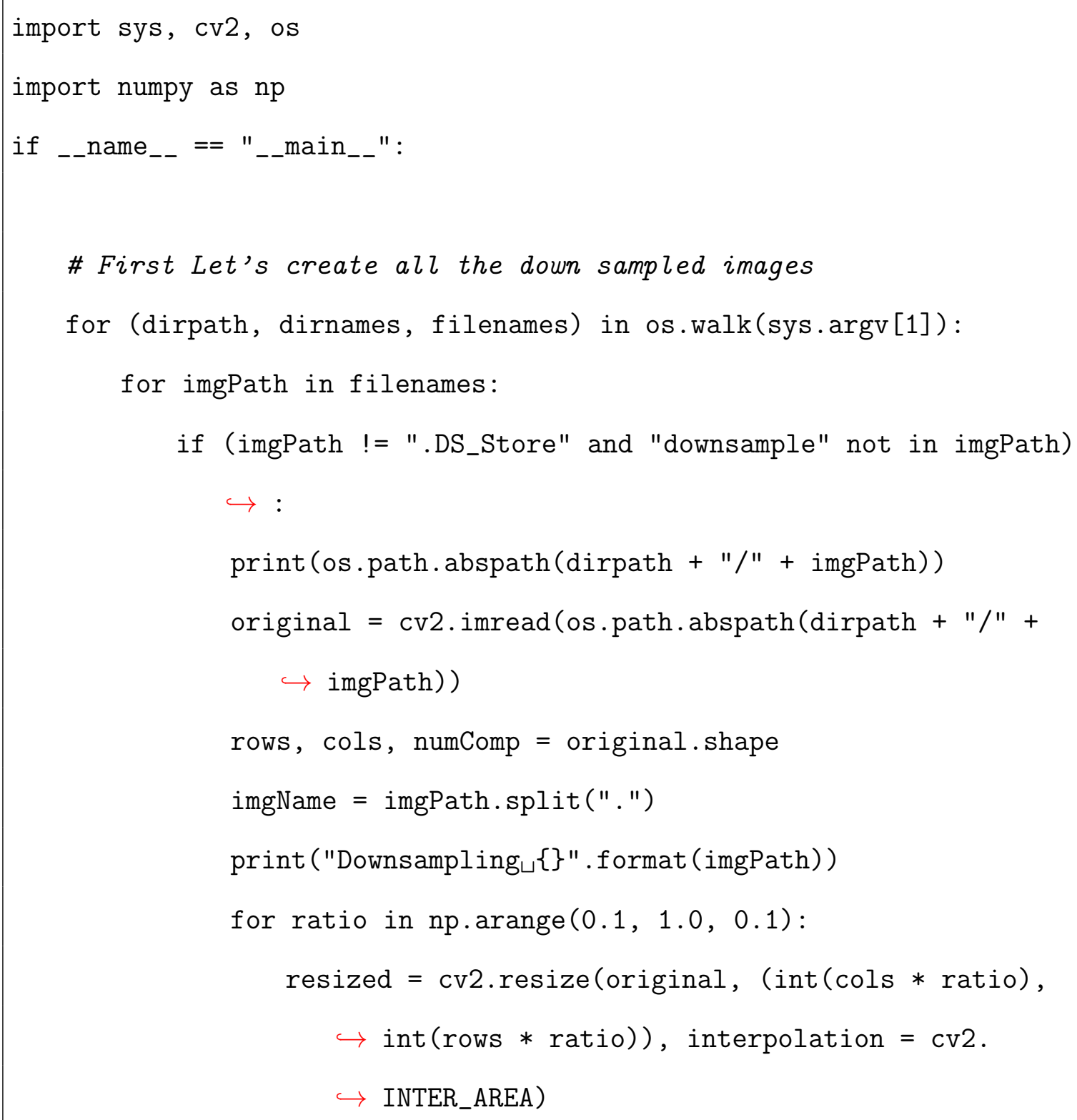


cv2.imwrite("./Downsampled/\{\}_downsample_\{\}. \{\}$"$.

$\hookrightarrow$ format ( imgName [0], int(ratio $* 10)$, imgName

$\hookrightarrow[1])$, resized)

break

\section{A.2 Analyze}

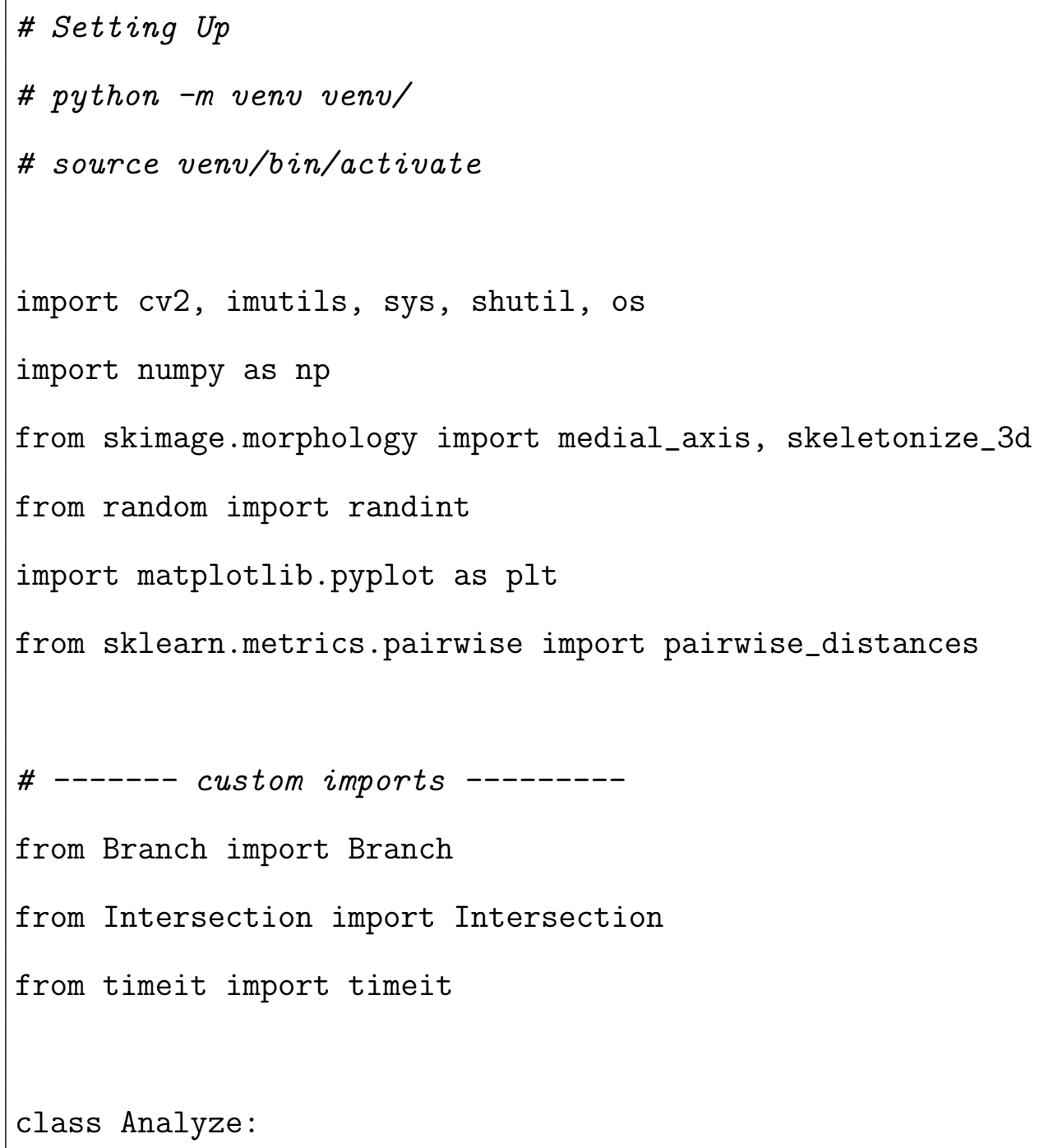

Qtimeit 


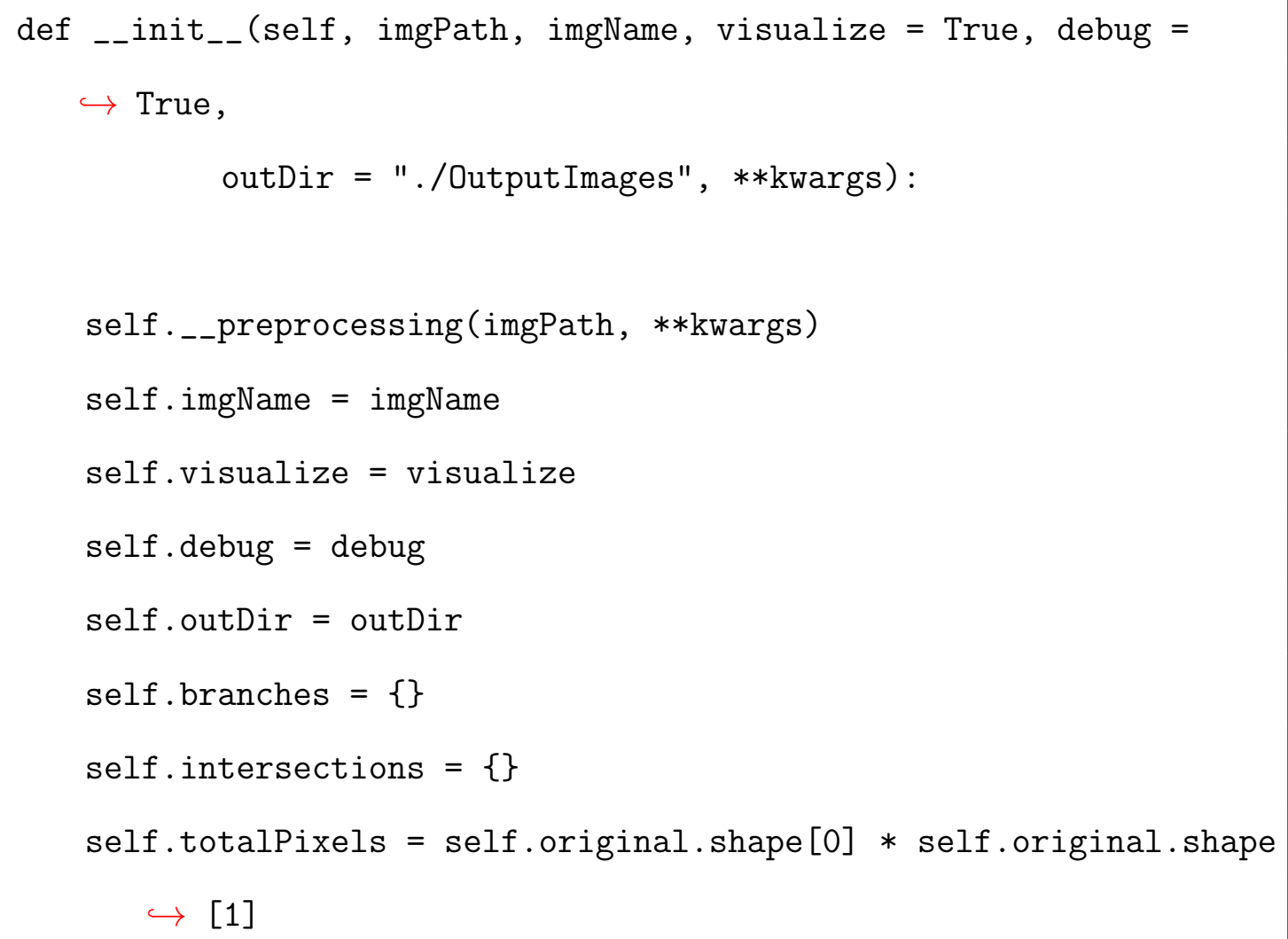




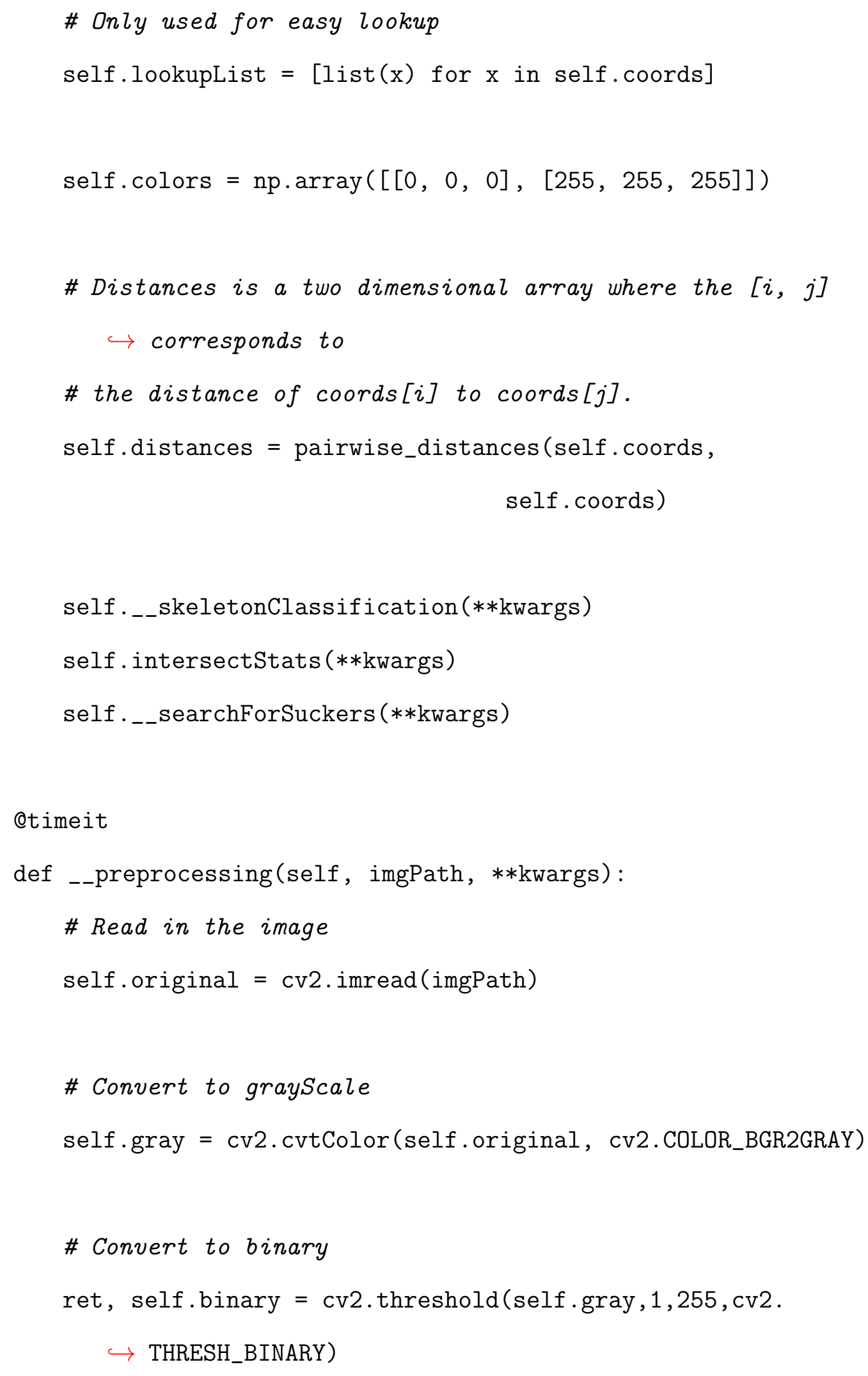




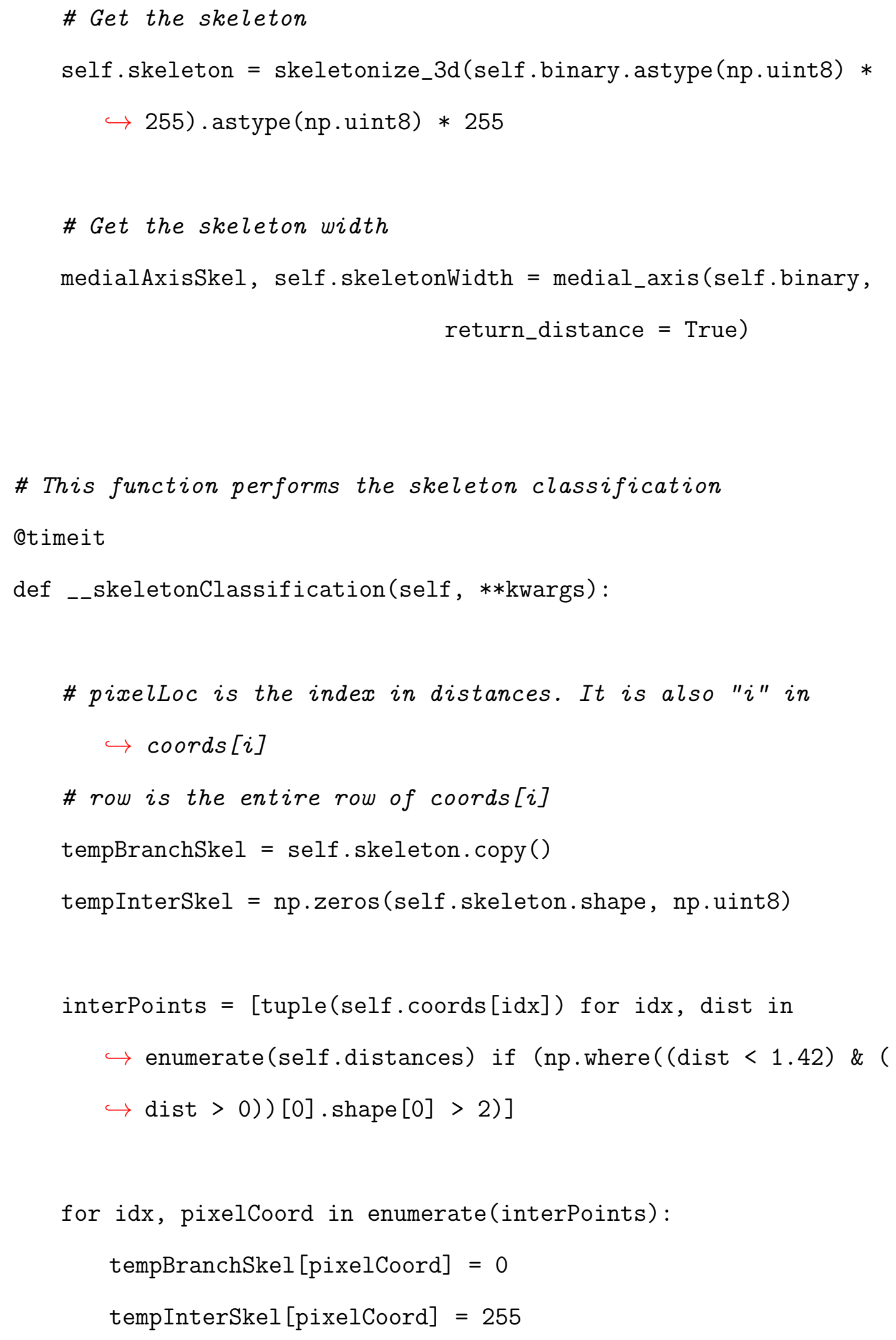


\# Classifying Pixels into branches

ret, self.labels $=c v 2$. connectedComponents (tempBranchSkel)

for label in np.unique(self.labels):

if (label):

self.branches [label] = Branch (label, self)

branchLabels $=n p$. unique $($ self .1 abels $)[1:]$

\# Classifying Pixels into Intersection Points

ret, tempInterLabels = cv2. connectedComponents (tempInterSkel,

$\hookrightarrow$ connectivity $=8$ )

for label in np.unique(tempInterLabels):

if $(\mathrm{abel})$ :

newLabel $=$ label + branchLabels $[-1]$

self.labels[tempInterLabels $==$ label] = newLabel

self.intersections [newLabel] = Intersection(newLabel, $\hookrightarrow$ self)

interLabels $=\mathrm{np}$. unique $($ self $.1 \mathrm{labels})[$ len $($ branchLabels $)+1:]$

\# Debug and Visualize

if (self.debug):

print ("After $\sqcup \_-$skeletonClassification..." )

print ("branchLabels: \{\} ".format (self.branches.keys ()))

print("interLabels: $\{\{\}$ ".format (self.intersections.keys())) 


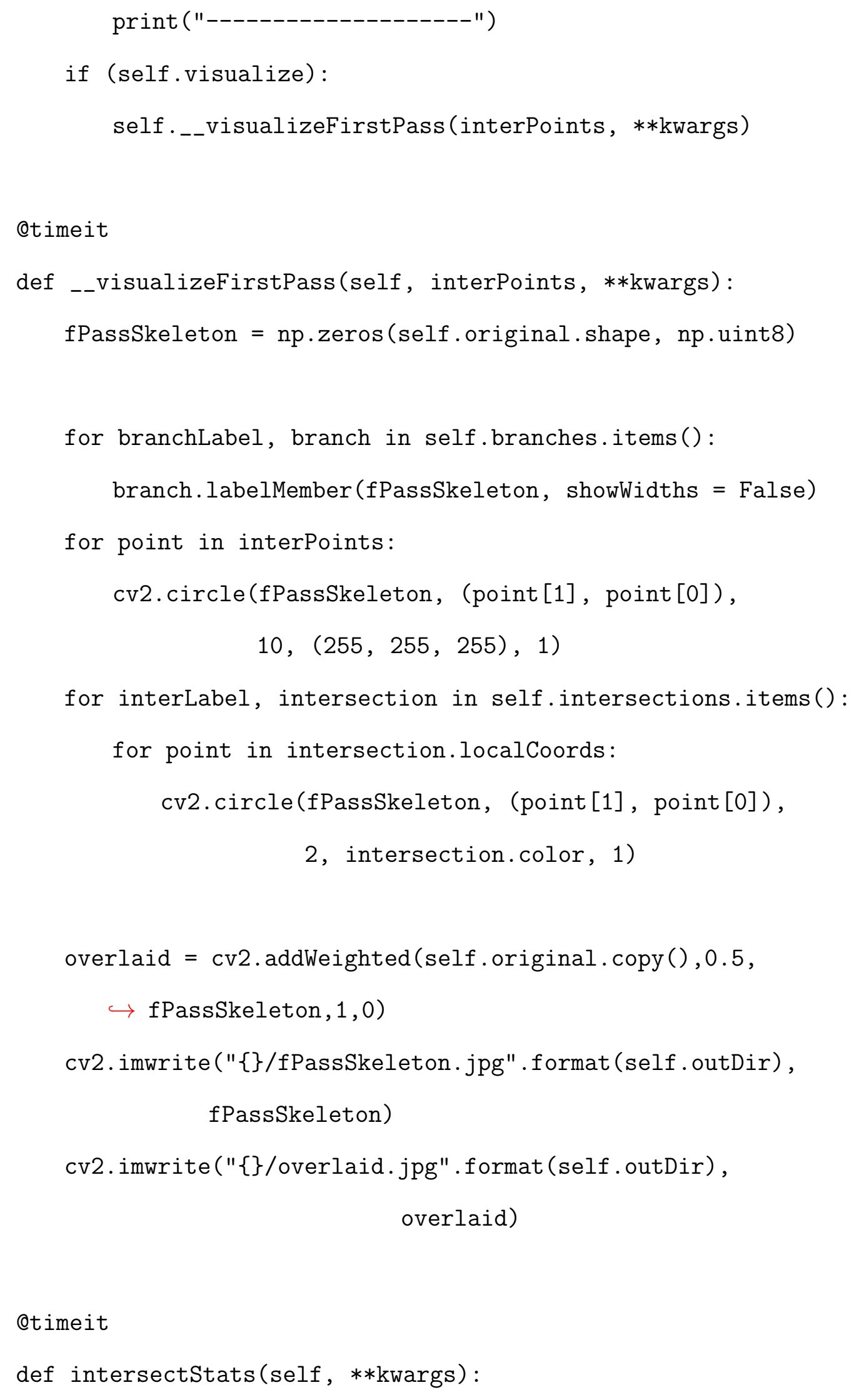




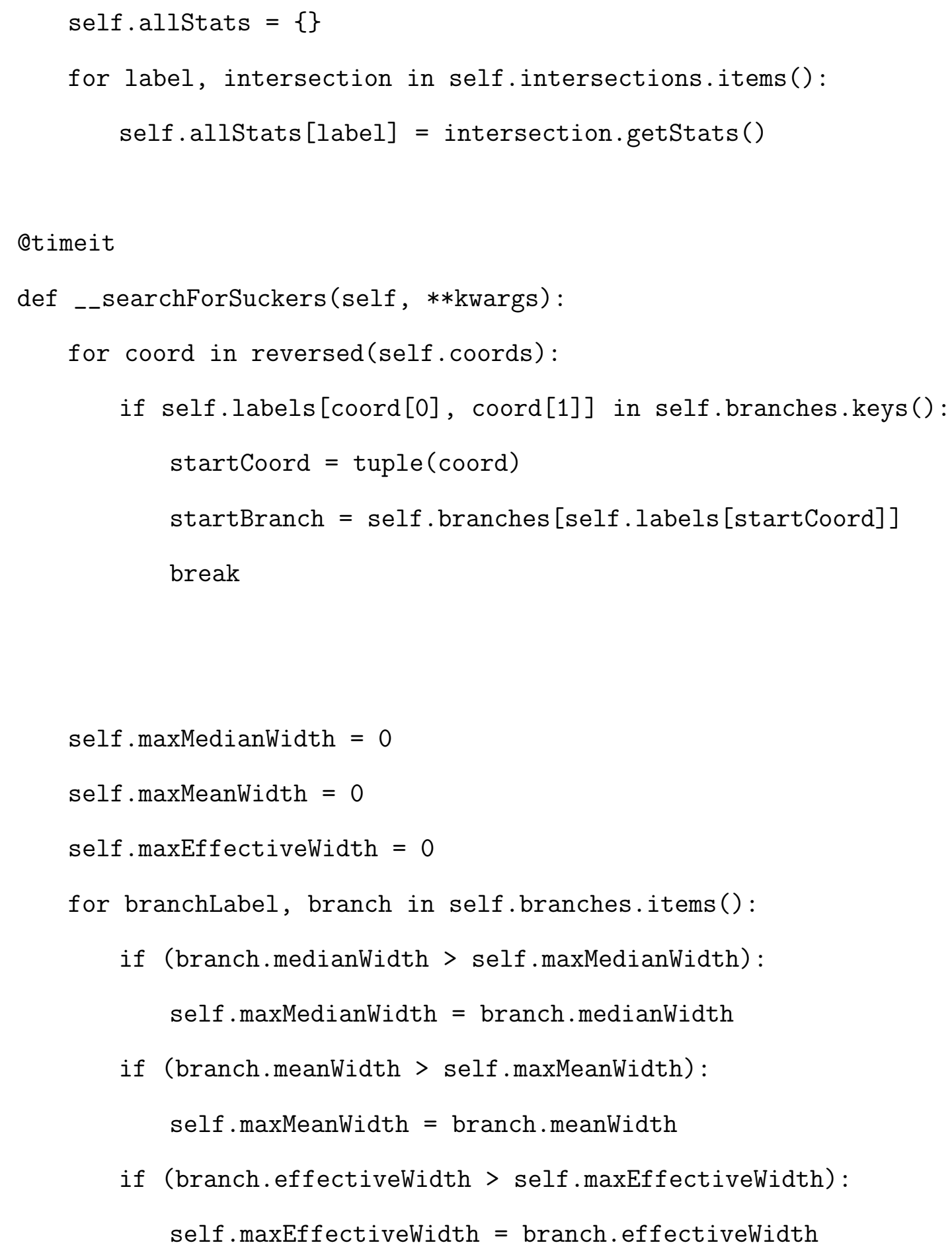


\# intersection point. This requires finding the nearest

$\hookrightarrow$ intersection

\# point that has a less y coord value than start coord.

startBranch. $\operatorname{crawlUp}()$

\#Visualize and debugging -------

if (self.debug):

self .printLabels("_searchForSuckers")

if (self.visualize):

self._-visualizeSearchForSuckers (**kwargs)

\section{Qtimeit}

def __visualizeSearchForSuckers (self, **kwargs):

for branchLabel, branch in self.branches.items():

branch.drawWidth()

tempSkeleton $=n p \cdot$ zeros $($ self. original. shape, np.uint8)

for branchLabel, branch in self.branches.items():

if (branchLabel in [1]): tempSkeleton [self.labels == branch] = branch.color

cv2.imwrite("\{\}/\{\}_marked.png".format(self.outDir, self.imgName

$\hookrightarrow)$

self . prunedImg) 
def printLabels (self, phase):

print ("After $\sqcup\{\} \ldots$...format (phase))

print ("branchLabels: \{\} ". format (self.branches.keys()))

print("interLabels: \{\} ".format (self.intersections.keys()))

print ("-------------------- ")

if __name__ == "_-_main_-_":

imgPath = sys.argv[1]

clear = sys.argv[2] == "True"

path = imgPath.split("/")

imgName $=\operatorname{path}[-1] . \operatorname{split}(" . ")[0]$

resultsFolder $=$ "./Results/" + imgName + "_results"

log_time_data $=\{\}$

if (clear):

shutil.rmtree(resultsFolder, ignore_errors = True)

elif (os.path.exists (resultsFolder)):

sys.exit()

os.makedirs (resultsFolder)

Analyze (imgPath, imgName, outDir = resultsFolder, visualize $=$ True

$\hookrightarrow$, debug $=$ True, $\log _{-}$time = log_time_data $)$

log_time_data['__skeletonClassification'] -= log_time_data['

$\hookrightarrow{ }_{-}$-visualizeFirstPass']

log_time_data['__searchForSuckers'] -= log_time_data['

$\hookrightarrow$ _-visualizeSearchForSuckers'] 


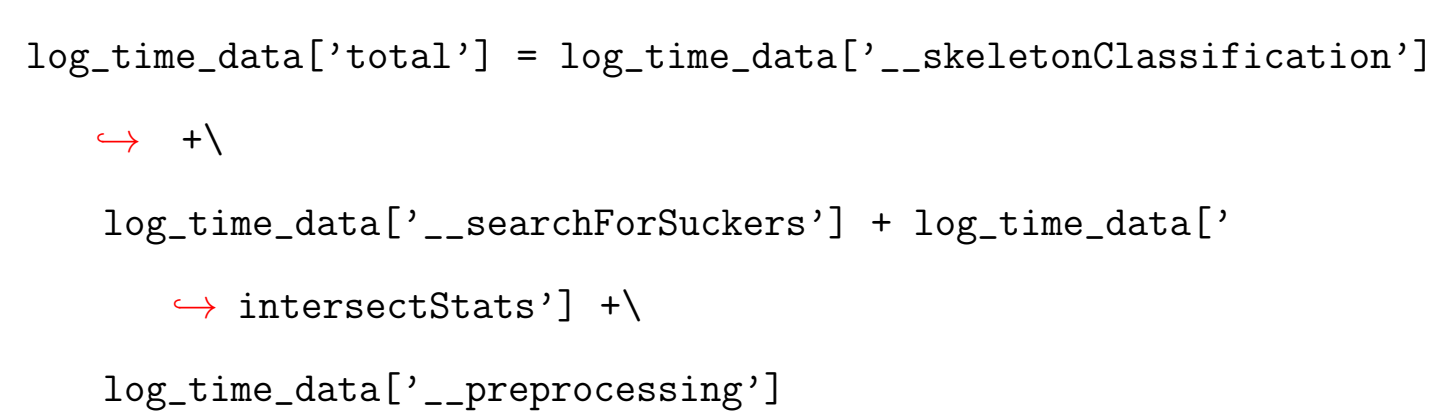

\section{A.3 Member}

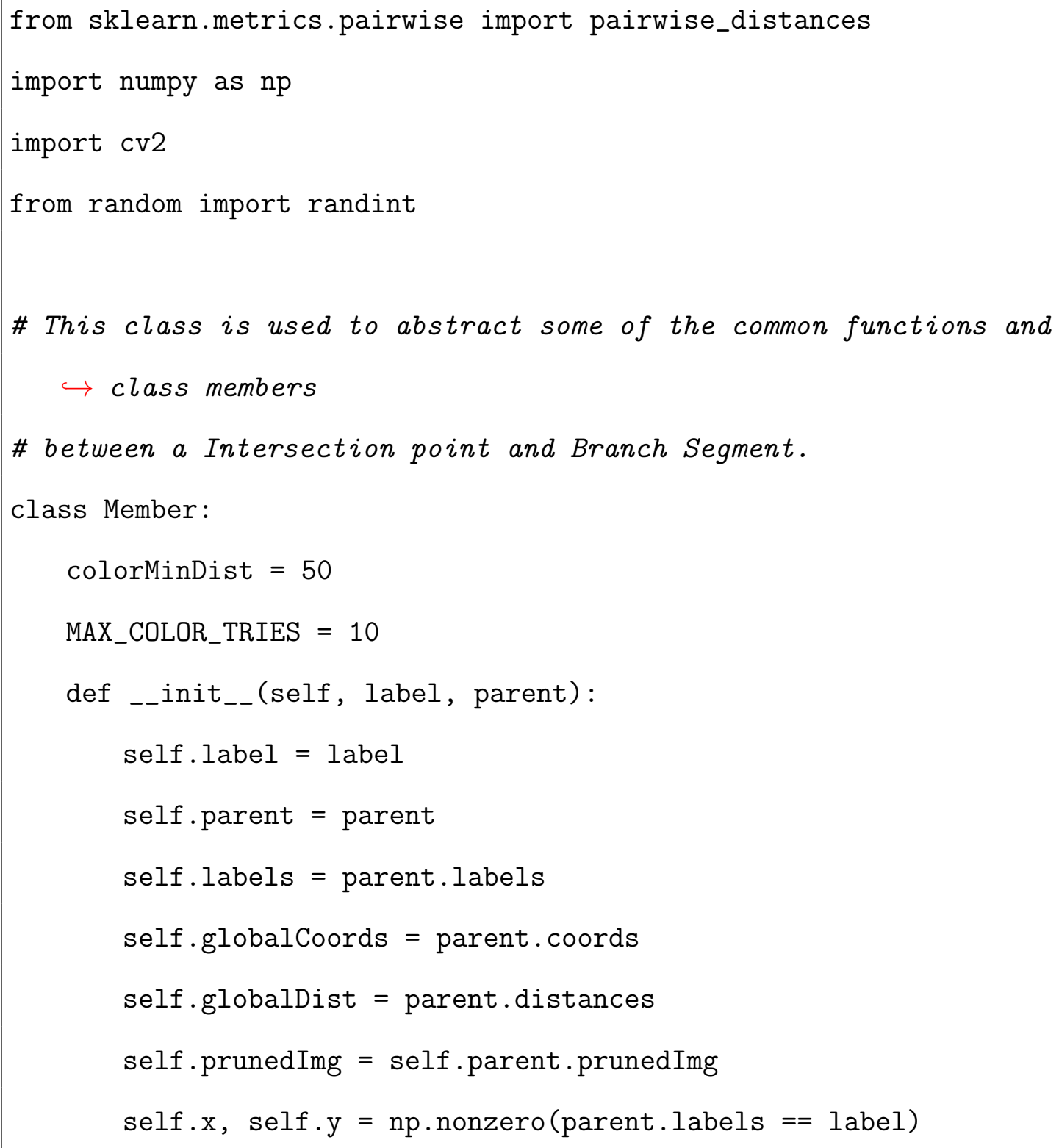




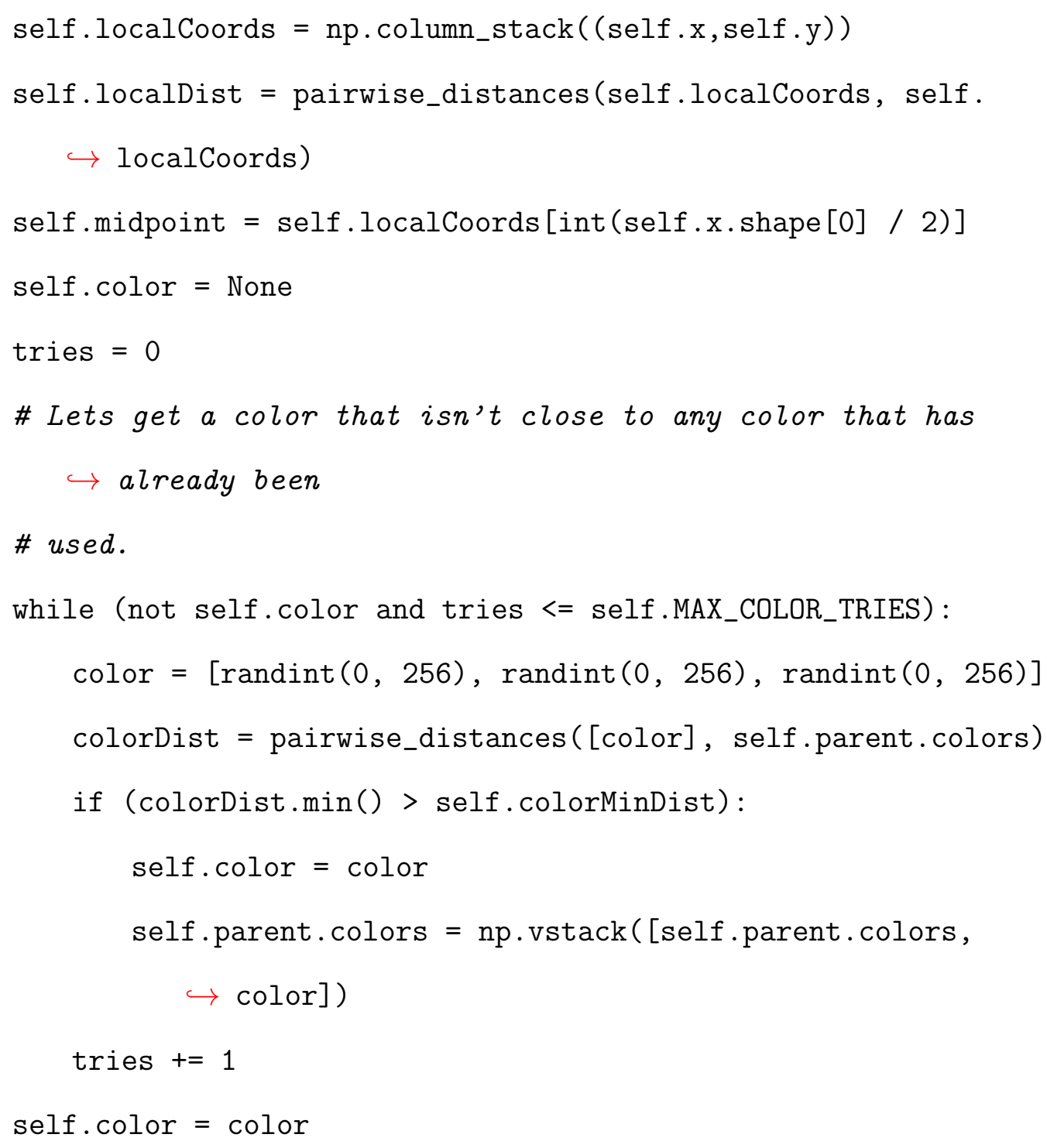

\# Gets the closest point between two members.

def getClosestPoint(self, other):

if (not issubclass (type(other), Member)):

print ("Not $\mathrm{a}_{\sqcup}$ Member. $\sqcup$ Type $\sqcup$ returned: $\sqcup\{\}$ ". format (type (other)) $\hookrightarrow$ )

raise TypeError()

dist $=$ pairwise_distances $($ self.localCoords, other.localCoords $)$ 
localIdxs, otherIdxs $=\mathrm{np} \cdot$ where $($ dist $==$ dist $\cdot \min ())$

return self.localCoords[localIdxs[0]], other.localCoords[

$\hookrightarrow$ otherIdxs[0]]

\# Gets the angle at which this member (branch) is intersecting a \# particuliar point

def getAngle(self, point, startRadius $=40$, endRadius $=1)$ :

dist $=$ pairwise_distances $(n p \cdot \operatorname{array}([$ point $])$, self.localCoords $)$

for radius in reversed(range(endRadius, startRadius)):

idxs $=$ np. where $(($ dist $>=$ radius $) \&($ dist $<=\operatorname{radius~}+1))[1]$

if (idxs.shape [0]):

ownPoint $=$ tuple $($ self. localCoords $[$ idxs $[0]])$

return $\mathrm{np} \cdot \operatorname{rad} 2 \mathrm{deg}(\mathrm{np} \cdot \arctan 2($

point [1] - ownPoint [1],

point [0] - ownPoint [0]))

\# Used for labeling a member with the width and label value on a

$\hookrightarrow$ skeleton

def labelMember(self, img,

font $=$ cv2.FONT_HERSHEY_SIMPLEX, fontScale $=0.5$,

lineType $=2$, showWidths $=$ True):

$\operatorname{img}[\operatorname{self} . x, \operatorname{self} . y]=\operatorname{self} . \operatorname{color}$

text $=\operatorname{str}($ self.label $)$

if (showWidths):

text $+=$ "பWidth: $\sqcup\{\} "$. format (int (self.meanWidth)) 
textW, textH = cv2.getTextSize(text, font, fontScale, lineType) $\hookrightarrow[0]$

bottomLeft $=(\operatorname{self} \cdot \operatorname{midpoint}[1]$, self.midpoint [0] + int (textH / 2))

cv2.putText(img, text, bottomLeft, font, fontScale, self.color, lineType)

\section{A.4 Branch}

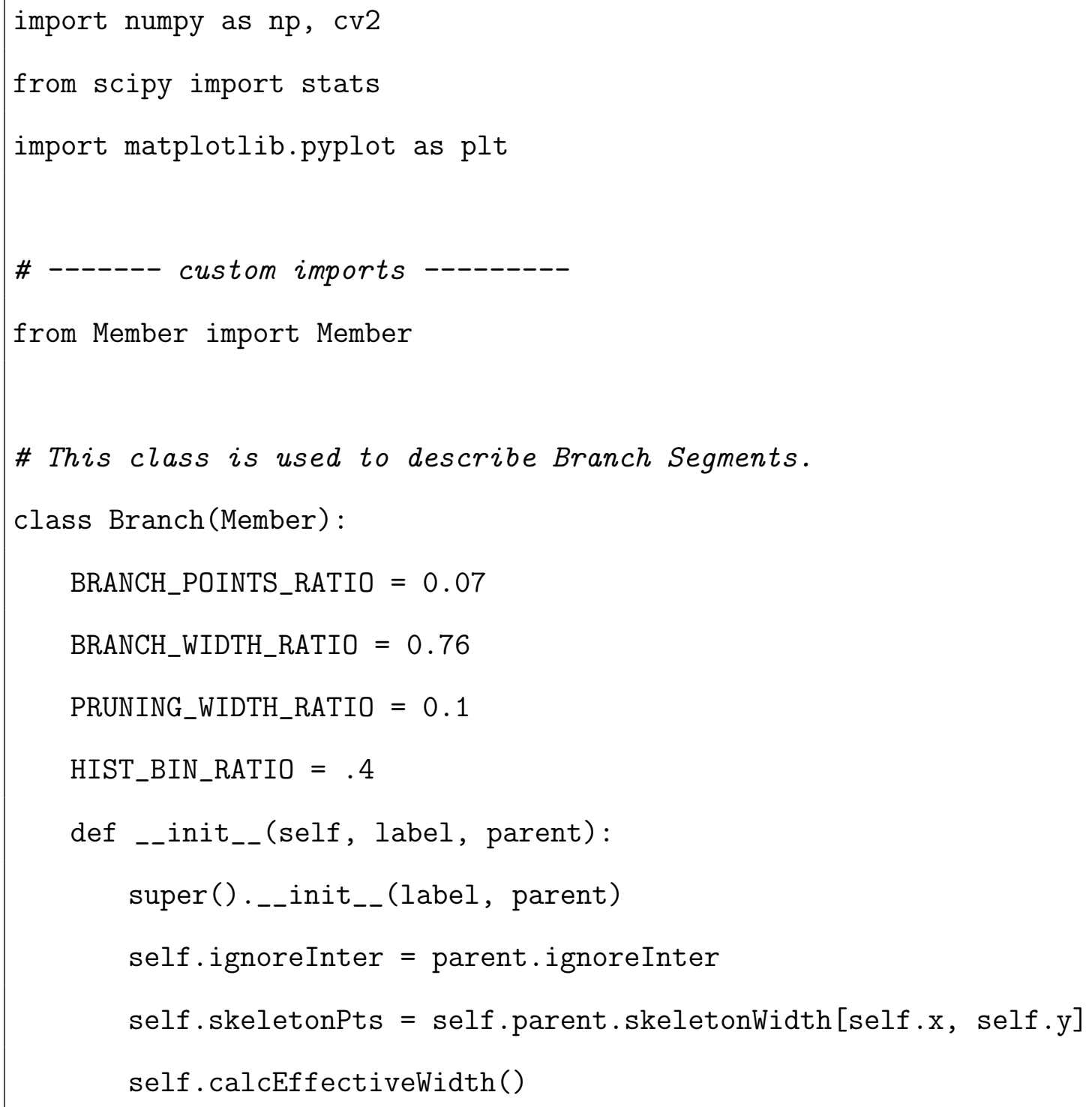


def calcEffectiveWidth(self):

self $\cdot$ meanWidth $=$ self $\cdot$ skeletonPts.mean ()

self. medianWidth $=$ np.median $($ self. skeletonPts $)$

self.widthHist, self.binEdges = np.histogram(self.skeletonPts,

$\hookrightarrow$ bins="auto")

mode, counts $=$ stats.mode $($ self. parent. skeletonWidth $[$ self.x,

$\hookrightarrow \operatorname{self} \cdot y])$

self $\cdot$ modeWidth $=\operatorname{mode}[0]$

top2Bins $=$ self.widthHist.argsort ()$[-2:][::-1]$

if (len(top2Bins) $==2$ and self.widthHist[top2Bins[0]] - self.

$\hookrightarrow$ widthHist[top2Bins[1]] > self.widthHist[top2Bins[1]] *

$\hookrightarrow$ self.HIST_BIN_RATIO) :

widthRange $=$ self.binEdges [top2Bins [0]: top2Bins[0] +2 ]

self.effectiveWidth $=$ widthRange $\cdot$ mean ()

else:

self.effectiveWidth $=$ self.meanWidth

\# Returns the intersection stats for this branch. Excludes this

$\hookrightarrow$ branch from

\# the stats returned.

def getRelevantStats (self):

for interLabel, stats in self.parent.allStats.items(): 
if (interLabel not in self.ignoreInter):

for index, branchInfo in enumerate(stats):

if (branchInfo['branchLabel'] == self.label):

refAngle = branchInfo['angle']

statsCopy $=\operatorname{stats}[:]$

statsCopy.pop (index)

return \{"interLabel": interLabel, "stats":

$\hookrightarrow$ statsCopy $\}$, refAngle

return $\{$ interLabel" : None, "stats": []\}, None

\# Starts crawling up the branch to the next IP. Once an IP point

$\hookrightarrow$ is reached,

\# the intersectings BSs are inspected for BSOI.

def crawlUp(self, branchOfInterest $=$ False, isRightBranch $=$ False, otherWidth $=0$, source $=$ None):

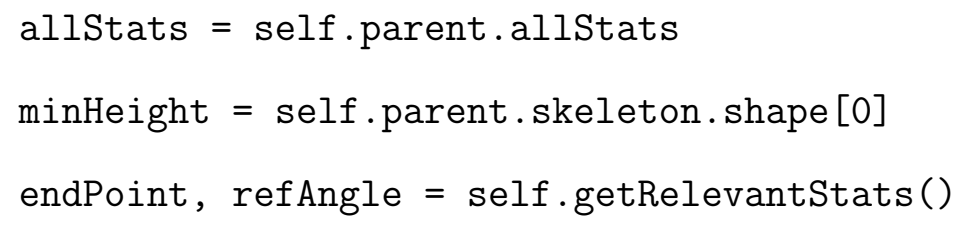

\# If there is no endpoint, return. This marks the end of a $\hookrightarrow$ branch

if (not endPoint['interLabel']):

return

\# Remove this intersection label so its not parsed again 
self.ignoreInter.append (endPoint ['interLabel'] )

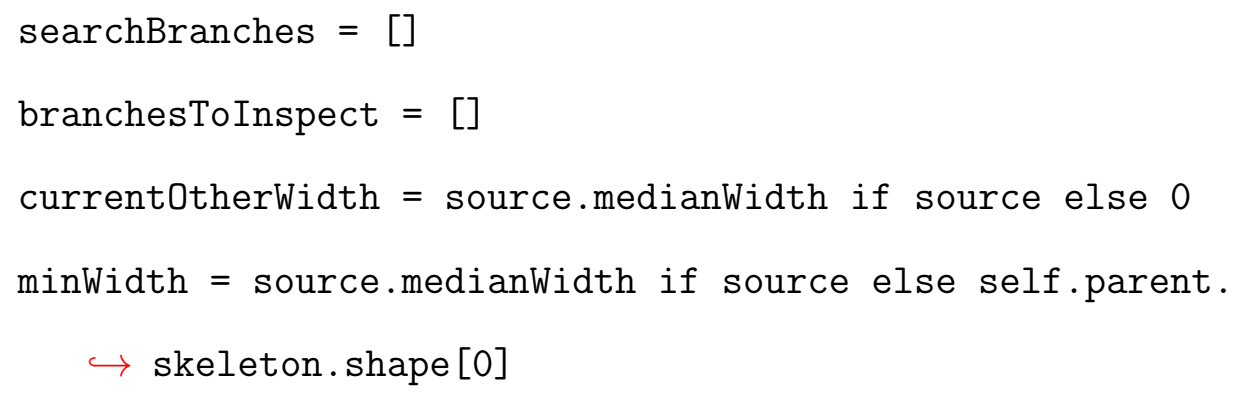


if (branchInfo['width'] < minWidth):

minWidth $=$ branchInfo['width']

minBranch $=$ self. parent. branches [branchInfo ['

$\hookrightarrow$ branchLabel']]

currentOtherWidth $=$ branchInfo['width']

searchBranches . append (branch)

\# Sort the branches by angle diff. The lesser angle is

$\hookrightarrow$ considered

\# the right branch

endPoint ['stats']. sort (key=lambda x:x['angleDiff'])

if (len (branchesToInspect)):

for branch in branchesToInspect:

newSource $=$ source

if (not source):

newSource $=$ minBranch if minBranch else self

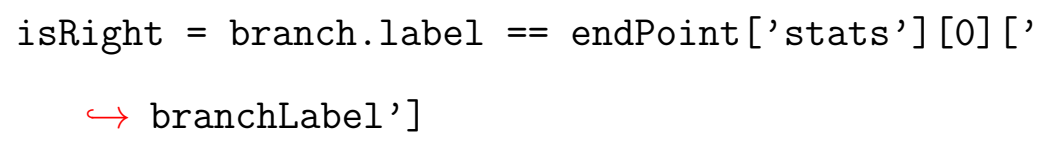

\# This means this branch is a branch of interest and no other

$\hookrightarrow$ branches

\# of interest are connected to this branch. This means we

$\hookrightarrow$ need to check

\# for a sucker 
if (branchOfInterest):

possibleSuckerIdx $=1$ if isRightBranch else 0

nonSuckerIdx = 0 if isRightBranch else 1

possibleSucker $=$ endPoint ['stats'] [possibleSuckerIdx]

possibleSuckerBranch = self $\cdot$ parent.branches $[$ possibleSucker [

$\hookrightarrow$ 'branchLabel']]

nonSucker $=$ endPoint ['stats'] [nonSuckerIdx]

nonSuckerBranch $=$ self . parent. branches [nonSucker ['

$\hookrightarrow$ branchLabel']]

\# The branch next to the potential sucker is an interest

$\hookrightarrow$ branch

\# We need to climb this interest

if (nonSuckerBranch in branchesToInspect and source):

nonSuckerBranch $=$ source

nonSucker $=\{$ 'width': source.medianWidth, 'branchLabel'

$\hookrightarrow$ : source.label\}

if (possibleSuckerBranch in searchBranches):

if ( possibleSucker['width'] < nonSucker ['width'] or possibleSucker ['width'] < otherWidth):

searchBranches.remove (possibleSuckerBranch)

possibleSuckerBranch.prune (nonSucker ['branchLabel'], startingPoint $=$ possibleSuckerBranch .

$\hookrightarrow$ findStartingPoint()) 
\# This is a rare ocurrence, but it does happen. There are 3

$\hookrightarrow$ branch

\# segments entering this intersection point.

\# We are going to check the middle most branches to see if

$\hookrightarrow$ there width

\# is less than the width of the right and left branches

elif (len (searchBranches) > 2):

rightBranch = endPoint ['stats'] [0]

leftBranch = endPoint ['stats'] [-1]

for branch in endPoint['stats'] [1: -1$]$ :

compWidth $=$ branch ['width']

if (compWidth < leftBranch ['width'] or

compWidth < rightBranch ['width']):

pruneBranch $=$ self $\cdot$ parent. branches [branch ['

$\hookrightarrow$ branchLabel']]

pruneBranch. prune (branch ['branchLabel'], startingPoint $=$ pruneBranch .

$\hookrightarrow$ findStartingPoint())

\# We need to continue searching the other branches

for branch in searchBranches:

branch. $\operatorname{crawlUp}()$

def isBranch0fInterest (self):

branchCoords $=$ self.localCoords. shape [0] 
ratioToSkelPoints $=$ branchCoords / float (self.parent. coords .

$\hookrightarrow$ shape [0] )

widthRatio $=$ self.meanWidth $/$ self.parent.maxMeanWidth print ("branch: $\{\{\}, \sqcup w i d t h: \sqcup\{\}, \sqcup$ skel: $\sqcup\{\}$ ". format (self.label,

$\hookrightarrow$ widthRatio, ratioToSkelPoints))

return (ratioToSkelPoints <= self.BRANCH_POINTS_RATIO and widthRatio >= self.BRANCH_WIDTH_RATIO)

\# Gets the endpoint for marking of a sucker def findStartingPoint(self):

\# Start from the reverse of coords

for coord in reversed(self.localCoords):

ratio $=(\operatorname{self} \cdot$ parent.skeletonWidth $[\operatorname{coord}[0], \operatorname{coord}[1]]-\backslash$ self.effectiveWidth) / self

$\hookrightarrow$.effectiveWidth

if (ratio $>=0$ and ratio $<=$ self.PRUNING_WIDTH_RATIO): return coord

\# Marks this branch red and any other branches connected to it. def prune(self, ignoreBranch $=$ None, startingPoint $=$ None $)$ :

endPoint, refAngle $=$ self.getRelevantStats ()

if (endPoint ['interLabel']):

self.ignoreInter. append (endPoint ['interLabel'])

\section{\# Recursive prune}

for branchInfo in endPoint['stats']: 
branch $=$ self $\cdot$ parent. branches [branchInfo['branchLabel'] $]$

bEnd, refAngle = branch.getRelevantStats()

if (ignoreBranch and not any(info['branchLabel'] ==

$\hookrightarrow$ ignoreBranch for info in bEnd['stats'])):

branch.prune (ignoreBranch)

\# Now we have to get all the coordinates of this branch so we

$\hookrightarrow$ can mark

\# it red on the original image for pruning

if (startingPoint is not None):

endingIndex $=[$ list $(\mathrm{x})$ for $\mathrm{x}$ in self.localCoords $]$. index (

$\hookrightarrow \operatorname{list}($

startingPoint

$\hookrightarrow)$ )

else:

endingIndex $=$ self.localCoords.shape[0]

for coord in self.localCoords [: endingIndex]:

$\operatorname{coord}=\operatorname{tuple}(\operatorname{coord})$

angle $=$ self $\cdot$ getAngle $($ coord, startRadius $=3)$

width $=\operatorname{int}($ self $\cdot$ parent.skeletonWidth $[$ coord $])$

if (angle) :

$\mathrm{xComp}=\operatorname{int}(\mathrm{np} \cdot \cos ($ angle $) *$ width $)$

yComp $=\operatorname{int}(n p \cdot \sin ($ angle $) *$ width $)$

startX $=\operatorname{coord}[0]-\mathrm{xComp}$

endX $=\operatorname{coord}[0]+\mathrm{xComp}$

startY $=\operatorname{coord}[1]-\mathrm{yComp}$

endY $=\operatorname{coord}[1]+\mathrm{yComp}$ 
if $($ startX > endX) :

temp $=\operatorname{startX}$

startX $=$ endX

endX $=$ temp

if (startY > endY):

temp $=$ startY

startY $=$ endY

endY $=$ temp

else:

$$
\begin{aligned}
& \text { startX }=\operatorname{coord}[0] \\
& \text { endX }=\operatorname{coord}[0]+1 \\
& \text { startY }=\operatorname{coord}[1]-\text { width } \\
& \text { endY }=\operatorname{coord}[1]+\text { width }
\end{aligned}
$$

self.prunedImg [startX: endX, startY: endY $]=[0,0,255]$

\# Used for outputting the histograms for each branch segment def drawWidth(self):

plt.hist (self.parent.skeletonWidth[self.x, self.y], bins="auto" $\hookrightarrow$,

color=' c', edgecolor='k', alpha $=0.65$ )

plt.axvline (self.meanWidth, color='k', linestyle='dashed', linewidth=1)

plt.title("Histogram for $_{\sqcup}$ branch $_{\sqcup}\{\} \backslash$ Mean $\sqcup$ width $: \sqcup\{\}, \sqcup M$ dian $\sqcup$

$\hookrightarrow$ width: $\{\{\} "$ format(self.label, round(self.meanWidth, 3 ), round (self.medianWidth, 3)))

plt.savefig("\{\}/branch\{\}Hist.png".format (self .parent.outDir, self.label)) 
plt.clf ()

\section{A.5 Intersection}

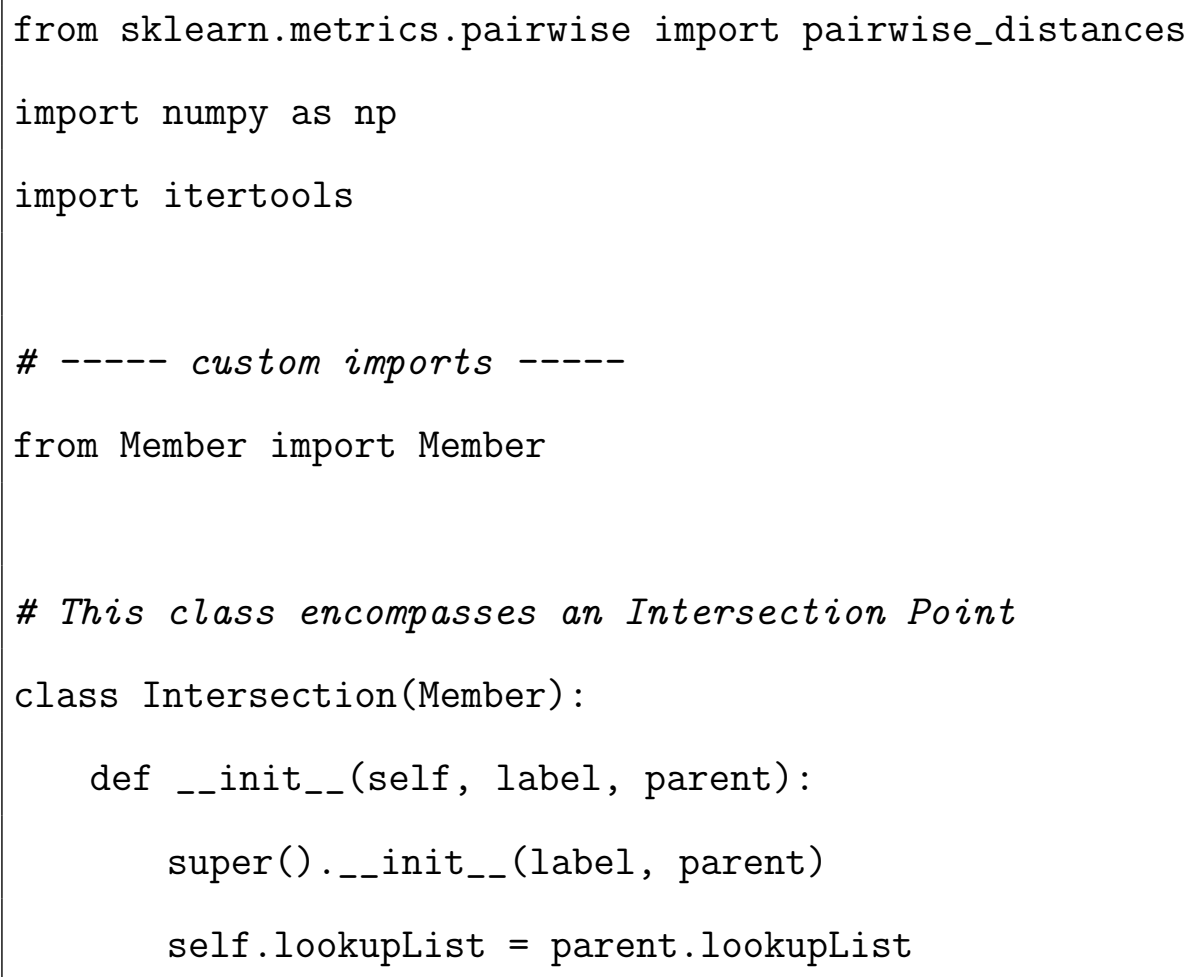




$$
(\text { row }>0))[0]
$$

for nCoord in self.globalCoords [nIdxs]:

nLabel $=\operatorname{self.labels}[$ tuple $($ nCoord $)]$

branchInfo $=\{$

'branchLabel': nLabel,

'angle': None,

'width': None

\}

if (branchInfo not in stats and

nLabel not in self.parent.intersections):

stats .append (branchInfo)

\# Next we are going to get stats for each intersecting branch

$\hookrightarrow \quad l a b e l$

\# 1. First we need to find the closest intersection point to

$\hookrightarrow$ this branch

\# 2. Then we need to get the angle at which this branch is

$\hookrightarrow$ coming into

\# the intersection point

for branchInfo in stats:

branchLabel $=$ branchInfo['branchLabel']

branch $=$ self $\cdot$ parent. branches [branchLabel]

interPoint, branchPoint $=$ self.getClosestPoint(branch)

branchInfo['angle'] = branch.getAngle(interPoint)

branchInfo['width'] = branch.effectiveWidth

return stats 